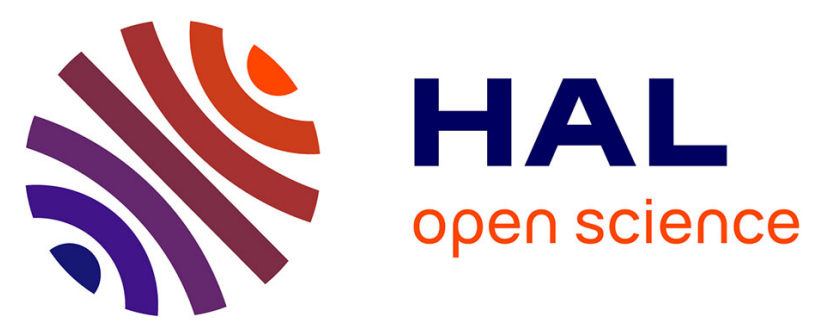

\title{
Geometric optics expansions for hyperbolic corner problems II : from weak stability to violent instability
}

Antoine Benoit

\section{To cite this version:}

Antoine Benoit. Geometric optics expansions for hyperbolic corner problems II : from weak stability to violent instability. SIAM Journal on Mathematical Analysis, In press, 49 (5), pp.3335-3395. 10.1137/16M1060145. hal-01242899v4

\section{HAL Id: hal-01242899 \\ https://hal.science/hal-01242899v4}

Submitted on 9 Mar 2017

HAL is a multi-disciplinary open access archive for the deposit and dissemination of scientific research documents, whether they are published or not. The documents may come from teaching and research institutions in France or abroad, or from public or private research centers.
L'archive ouverte pluridisciplinaire HAL, est destinée au dépôt et à la diffusion de documents scientifiques de niveau recherche, publiés ou non, émanant des établissements d'enseignement et de recherche français ou étrangers, des laboratoires publics ou privés. 
1ex plus .25ex minus .1ex 1ex plus .25ex minus .1ex

\title{
GEOMETRIC OPTICS EXPANSIONS FOR HYPERBOLIC CORNER PROBLEMS II : FROM WEAK STABILITY TO VIOLENT INSTABILITY.
}

\author{
ANTOINE BENOIT*
}

\begin{abstract}
In this article we are interested in the rigorous construction of geometric optics expansions for weakly well-posed hyperbolic corner problems. More precisely we focus on the case where selfinteracting phases occur and where one of them is exactly the phase where the uniform Kreiss-Lopatinskii condition fails. We show that the associated WKB expansion suffers arbitrarily many amplifications before a fixed finite time. As a consequence, we show that such a corner problem can not be weakly well-posed even at the price of a huge loss of derivatives. The new result, in that framework, is that the violent instability (or Hadamard instability) does not come from the degeneracy of the weak Kreiss-Lopatinskii condition, but of the accumulation of arbitrarily many weak instabilities.
\end{abstract}

Key words. hyperbolic corner problems, geometric optics expansions, selfinteraction phases, violent instability

AMS subject classifications. 35L04, 78A05

1. Introduction. The main study in this article is the rigorous construction of geometric optics expansions for hyperbolic corner problem that is to say, problems reading :

$$
\left\{\begin{array}{l}
L(\partial) u:=\partial_{t} u+A_{1} \partial_{1} u+A_{2} \partial_{2} u=f, \text { for } x_{1}, x_{2}>0 \\
B_{1} u_{\mid x_{1}=0}=g_{1}, \text { on } x_{1}>0, \\
B_{2} u_{\mid x_{2}=0}=g_{2}, \text { on } x_{2}>0, \\
u_{\mid t \leq 0}=0,
\end{array}\right.
$$

where the matrices $A_{i} \in \mathbf{M}_{N}(\mathbb{R})$ and where $B_{i} \in \mathbf{M}_{p_{i} \times N}(\mathbb{R})$ (the values of $p_{1}$ and $p_{2}$ will be made precise in Assumption 2).

Under the uniform Kreiss-Lopatinskii condition

$$
\operatorname{ker} B_{1} \cap E_{1}^{s}(\zeta)=\{0\}, \text { and } \operatorname{ker} B_{2} \cap E_{2}^{s}(\zeta)=\{0\}
$$

where the $E_{1}^{s}(\zeta)$ and $E_{2}^{s}(\zeta)$ are stable subspaces depending of a set of frequency parameters $\zeta$ (see Definition 7 for a more precise definition). It is known that the associated initial boundary value problem in the half space is strongly well-posed (in the sense that we have existence and uniqueness of the solution and that this solution is as regular (in the $L^{2}$-norm) as the source terms in (1)) if and only if the uniform Kreiss-Lopatinskii condition is satisfied.

The analogous result have not, in the author knowledge, been demonstrated yet for initial boundary value problems in the quarter space. However the results of [17]-[18] indicate that an extra condition of invertibility will be needed in the quarter space geometry and thus that imposing the uniform Kreiss-Lopatinskii condition on each side of the boundary will not be sufficient to ensure strong well-posedness.

However even if the well-posedness is not establish for corner problems, the geometric optics expansions have been studied in [19] and more recently by the author in [3] and [4]. More precisely in [19] the authors give precious intuitions and some elements of proof about this construction which are used in [3]-[4] to construct rigorously the expansions.

In particular a new phenomenon, proper to the corner problem, has been investigated, the selfinteraction phenomenon. In more details, this phenomenon induces that some phases in the WKB expansion can generate themselves after a suitable number of reflections on the sides of the quarter space (see [3]-[4]). That is to say that some information lying on the side $\left\{x_{1}=0, x_{2}>0\right\}$ can be transported towards the side $\left\{x_{1}>0, x_{2}=0\right\}$, be reflected in the phase that send back the information towards $\left\{x_{1}=0, x_{2}>0\right\}$ and the same process is then repeated one more time. However it is possible that during the fourth rebound the obtained phase is exactly the one that was initially considered. So this phase will be generated several times. This was not the case for the half space geometry because a reflected phase automatically espaces to infinity. On one hand, in terms of the geometry of the characteristic variety, such systems contain a rectangle whose corners are elements of the characteristic variety (with suitable group velocities) and whose side are parallel

*Université Libre de Bruxelles, Département de Mathématiques, boulevard du triomphe 1050 Bruxelles Belgique Projectteam MEPHYSTO, Inria Lille - Nord Europe 40, avenue Halley, 59650 Villeneuve d'Ascq, France antoine.benoit@inria.fr, . 
to the axis of the frequency space. On the other hand, in terms of the resolution of the WKB cascade, a new amplitude equation, whose provenance is intrinsically linked with the uniform Kreiss-Lopatinskii condition has to be solved to initialize the resolution of the cascade.

We are here interested in corner problems whose, one of the boundary condition, to fix the ideas, let us say $B_{1}$, does not satisfy the uniform Kreiss-Lopatinskii condition that is that there exists at least one frequency parameter $\underline{\zeta}$ such that:

$$
\operatorname{ker} B_{1} \cap E_{1}^{s}(\underline{\zeta}) \neq\{0\} .
$$

The other boundary condition is assumed to be as convenient as necessary, that is it satisfies the uniform Kreiss-Lopatinskii condition or even it is strictly dissipative see [6]. The litterature about the hyperbolic boundary value problem in the half space tells us that such problems can not be strongly well-posed because of the failure of the uniform Kreiss-Lopatinskii condition. However they can be weakly well-posed in the sense that we still have existence and uniqueness but that the solution of (1) is now less regular that the sources terms in (1). We also say that the solution suffers losses of derivatives.

The number of losses depends on the particular structure of $E_{1}^{s}(\underline{\zeta})$ at the considered $\underline{\zeta}$. There are four possible kinds of degeneracy of the uniform Kreiss-Lopatinskii condition relying on the structure of $E_{1}^{s}(\underline{\zeta})$ at the frequency where the uniform Kreiss-Lopatinskii condition breaks down (see Definition 4). Namely, the degeneracies of the uniform Kreiss-Lopatinskii condition can occur in the so-called hyperbolic, elliptic, mixed or glancing regions. In terms of geometric optics expansions hyperbolic modes are associated to transport phenomenon whereas elliptic ones are associated to boundary layers.

Concerning boundary value problems in the half space for which the uniform Kreiss-Lopatinskii condition is violated, the construction of the associated geometric optics expansions has already been made in all but the glancing region. We refer to [8] for the construction when the degeneracy takes place in the hyperbolic region and to [2] and [14] for the construction associated to a degeneracy in the elliptic or in the mixed region. With this expansions it is then possible to saturate the energy estimates for the solution of (1) and to show that the obtained losses in the litterature are sharp.

We will here focus our attention on a degeneracy of the uniform Kreiss-Lopatinskii condition in the hyperbolic region, and thus for a frequency that induce a transport phenomenon. Such problems are refer as the $W R$ class in the sense of [5]. Some conjectures about the behaviours of the geometric optics expansions when the degeneracy of the uniform Kreiss-Lopatinskii condition occurs in the elliptic or in the mixed region can be found in Section 9.

In [8], the authors construct the geometric optics expansions for such $W R$ problems in the half space and show that if the source terms are of order one compared to the small parameter $\varepsilon$ which encodes the high oscillating behaviour, then the leading term in the geometric optics expansion is of order zero compared to $\varepsilon$. Then, they used this construction to show that the energy estimate with losses of derivatives established by [7]:

$$
\|u\|_{L^{2}\left(\Omega_{T}\right)}^{2}+\left\|u_{\mid x_{1}=0}\right\|_{L^{2}\left(\Omega_{T}\right)}^{2} \leq C_{T}\left(\|f\|_{L_{x_{1}}^{2}\left(H^{1}\left(\partial \Omega_{1, T}\right)\right)}^{2}+\left\|g_{1_{\mid x_{1}=0}}\right\|_{\left(H^{1}\left(\partial \Omega_{1, T}\right)\right)}^{2}\right)
$$

is sharp in terms of losses of derivatives.

Concerning corner problems, the litterature is much more poor. Indeed, as already mentionned the full characterization of strongly well-posed problems is, to our knowledge, not achieved yet. So, the full characterization of weakly well-posed corner problems seems, in the author opinion, to be a long range problem. As a consequence the fact that we construct the geometric optics expansions for these (expected to be) weakly well-posed problems can be seen as a "Majda's project" (see [1] and [13]). That is to say, that the author believies that the use of geometric optics expansions can give some intuition about what can be the good number of losses of derivatives in the associated corner problems.

In this article, we will focus our attention on the particular case of corner problems in the $W R$ where the frequency of degeneracy is one of the selfinteracting phases. Our purpose is thus to construct the geometric optics expansion for such a corner problem. As we will see in Section 7, for any arbitrarily large $M \in \mathbb{N}$, the fact that the phase for which the uniform Kreiss-Lopatinskii condition breaks down is generated an arbitrarily number of times will imply a leading term in the geometric optics expansion of order zero, compared with 
the small parameter $\varepsilon$, when one considers a source term on the boundary $\left\{x_{1}=0\right\}$ of order $M+1$ in terms of $\varepsilon$.

Using this geometric optics expansion, we will show that such a corner problem can not be weakly wellposed on a finite interval of time because it loses an arbitrarily large number of derivatives before the time $T$. In terms of losses of derivatives, it is the worst case that we can imagine because we have a violent or Hadamard's instability.

The paper is organized as follows : in Section 2, we precise the terms of the previous discussion on an example. The analysis made in Section 2 is purely formal and aims to illustrate the amplification and the self-interaction phenomenons. Then Section 3 gives some classical definitions and introduce some notations. In Section 4 we give a formal study of our problem and we describe what are the expected phases and the associated amplitudes in the WKB expansion. Then in Section 6, we give a general framework in which the previous formal discussion becomes rigorous. This section uses the same tools as these introduced in [4] to describe, with precision, the set of expected phases in the WKB expansion. However, we believe that it is important to recall these tools for the sake of completness.

Section 7 is devoted to the construction of the geometric optics expansion and is the main section of the paper. The most difficult part of the construction is to find a way to initialize the resolution of the WKB cascade of equations. Indeed in [4] a new amplitude equation whose resolution permits to initialize the WKB expansion was derived from the uniform Kreiss-Lopatinskii condition. However the obtention of this new equation is intrinsically linked with the uniform Kreiss-Lopatinskii condition. As a consequence to initialize the resolution of the WKB cascade when the uniform Kreiss-Lopatinskii condition degenerates, we need a new amplitude equation. This equation comes from an adaptation of the method described in [8]. The hardest point to handle with is that in [8], due to the "nice" geometry of the half space, it was possible to determine all the outgoing phases (which act as source terms in the equation determining the amplitude for which the uniform Kreiss-Lopatinskii breaks down) before all the others. However, this is not true anymore in the quarter space geometry. But we found a new equation for the initialization of the WKB, which one is given in paragraph 7.1. The resolution of this equation is made in subparagraphs 7.1.4 and 7.1.6. The resolution is made after a "necessary" reformulation of this equation, in view to show that this equation can in fact be rewritten under the particular form $(I-\mathbb{T}) u=G$, for some operator $\mathbb{T}$. This rewriting is made in paragraph 7.1.2. ${ }^{1}$

Section 8 contains the proof of our main result, that is that a corner problem in the $W R$ class for one side which admits a loop in the section of its characteristic variety and for which one of the element of the loop violates the uniform Kreiss-Lopatinskii condition can not be even weakly well-posed. It is not really a positive result because it tells us that, in such a framework, there is no hope to solve the corner problem. However, the author believes that this result is interesting in itself because it gives the first examples, in our knowledge, of ill-posed hyperbolic boundary value problems for which the ill-posedness is due to the accumulation of weak instabilities and not to the failure of the weak Kreiss-Lopatinskii condition. Moreover the instability phenomenon in this framework is much more subtle.

At last, Section 9 gives examples of such ill-posed corner problems and some (more optimistic) conjectures about what should be the leading order sizes (and so the number of losses of derivatives) in the more favorable cases where the uniform Kreiss-Lopatinskii condition breaks down outside the loop.

2. Example and formal discussion. We start this an illustrative example. We consider the following corner problem:

$$
\begin{cases}L(\partial) u^{\varepsilon}:=\partial_{t} u^{\varepsilon}+A_{1} \partial_{1} u^{\varepsilon}+A_{2} \partial_{2} u^{\varepsilon}=0, & \text { for } x_{1}, x_{2}>0 \\ B_{1} u_{\mid x_{1}=0}^{\varepsilon}=\varepsilon g^{\varepsilon}, & \text { for } x_{1}=0 \\ B_{2} u_{\mid x_{2}=0}^{\varepsilon}=0, & \text { for } x_{2}=0 \\ u_{\mid t \leq 0}^{\varepsilon}=0, & \text { for } x_{1}, x_{2}>0\end{cases}
$$

\footnotetext{
${ }^{1}$ Let us note that the particular structure $(I-\mathbb{T}) u=G$ was already the structure obtained in [4]. Moreover, this structure will be very important in the following proof because it permits to write the solution $u$ as the sum of compositions of $\mathbb{T}$ and thus to express the solution $u$ in terms of the sum of wave packets.
} 
where the coefficients in the interior are given by

$$
A_{1}:=\left[\begin{array}{ccc}
0 & \sqrt{5} & 0 \\
\sqrt{5} & -4 & 0 \\
0 & 0 & -\frac{5}{7}
\end{array}\right] \text {, and } A_{2}:=\left[\begin{array}{ccc}
-1 & 0 & 0 \\
0 & 1 & 0 \\
0 & 0 & 1
\end{array}\right] \text {. }
$$

And where the boundary matrices are given by:

$$
B_{1}:=\left[\begin{array}{lll}
-\sqrt{5} & \frac{7}{3} & 1
\end{array}\right], \text { and } B_{2}:=\left[\begin{array}{ccc}
-\frac{1}{\sqrt{5}} & 1 & 0 \\
-\frac{1}{\sqrt{5}} & 0 & 1
\end{array}\right] .
$$

The source term $g^{\varepsilon}\left(t, x_{2}\right):=e^{\frac{i}{\varepsilon}\left(t-8 x_{2}\right)} g\left(t, x_{2}\right)$, where $g$ is zero for negative times and is also zero for all $x_{2} \leq 1$.

Let $\mathscr{L}$ be the symbol of $L(\partial)$ it is defined for all $\left(\tau, \xi_{1}, \xi_{2}\right) \in \mathbb{R}^{3}$ by:

$$
\mathscr{L}\left(\tau, \xi_{1}, \xi_{2}\right):=\tau I+\xi_{1} A_{1}+\xi_{2} A_{2}
$$

It what follows it will be convenient to introduce the characteristic variety of $L(\partial)$, that is:

$$
V:=\left\{\left(\tau, \xi_{1}, \xi_{2}\right) \in \mathbb{R}^{3} \mid \operatorname{det} \mathscr{L}\left(\tau, \xi_{1}, \xi_{2}\right)=0\right\},
$$

and as the time frenquency in $g^{\varepsilon}$ will be constant equals to one, we introduce $\mathbf{V}:=V \cap\{\tau:=1\}$. The section $\mathbf{V}$ is given by:

$$
V_{\{\underline{\tau}=1\}}=\left\{\left(\xi_{1}, \xi_{2}\right) \in \mathbb{R}^{2} \backslash\left(1-\frac{5}{7} \xi_{1}+\xi_{2}\right)\left(1-4 \xi_{1}+4 \xi_{1} \xi_{2}-5 \xi_{1}^{2}-\xi_{2}^{2}\right)=0\right\},
$$

and is composed of an ellipse and a line (see Figure 1).

The aim of the following paragraph is to give a formal discussion based on the explicit example (2) illustrating the phenomenon of repetition of the instabilities mentioned in the introduction. The remaining of the article aims to give a rigorous justification of this formal discussion.

The corner problem (2) is hyperbolic so it has a finite speed of propagation. As a consequence the information supported by $g^{\varepsilon}$ can not hit immediately the boundary $\left\{x_{2}=0\right\}$. So in a very formal way, we will forget the quarter space geometry and work in the half space $\left\{x_{1} \geq 0, x_{2} \in \mathbb{R}\right\}$. The problem now reads:

$$
\begin{cases}\partial_{t} u^{\varepsilon}+A_{1} \partial_{1} u^{\varepsilon}+A_{2} \partial_{2} u^{\varepsilon}=0, & \text { for } x_{1} \in \mathbb{R}_{+}, x_{2} \in \mathbb{R}, \\ B_{1} u_{\mid x_{1}=0}^{\varepsilon}=\varepsilon g^{\varepsilon}, & \text { for } x_{1}=0, x_{2} \in \mathbb{R}, \\ u_{\mid t \leq 0}^{\varepsilon}=0, & \text { for } x_{1}>0, x_{2} \in \mathbb{R},\end{cases}
$$

and we are looking for an approximation solution of (3) via a WKB expansion.

We thus postulate exactly the same ansatz as in the half space:

$$
u^{\varepsilon} \sim \sum_{n \geq 0} e^{\frac{i}{\varepsilon} \varphi_{1}(t, x)} \varepsilon^{n} u_{1, n}+\sum_{n \geq 0} e^{\frac{i}{\varepsilon} \varphi_{4}(t, x)} \varepsilon^{n} u_{4, n}+\sum_{n \geq 0} e^{\frac{i}{\varepsilon} \varphi_{0}(t, x)} \varepsilon^{n} u_{0, n},
$$

where the phases functions $, \varphi_{0}, \varphi_{1}$ and $\varphi_{4}$ are given by: In particular, we remark that there is a loop with phases $\varphi_{1}, \ldots \varphi_{4}$ defined by:

$$
\varphi_{0}(t, x):=t-\frac{49}{5} x_{1}-8 x_{2}, \varphi_{1}(t, x):=t-\frac{21}{5} x_{1}-8 x_{2}, \text { and } \varphi_{4}(t, x):=t-3 x_{1}-8 x_{2},
$$

and we remark that the points $\left(-\frac{49}{5},-8\right),\left(-\frac{21}{5},-8\right)$ and $(-3,-8)$ are the three points of intersection between $\mathbf{V}$ and the line $\left\{\xi_{2}=8\right\}$. 
Plugging the ansatz (4) in the boundary value problem in the half space (3) gives the cascade of equations in the interior:

$$
\begin{cases}\mathscr{L}\left(d \varphi_{k}\right) u_{0, k}=0, & \text { for } k \in\{0,1,4\}, \\ i \mathscr{L}\left(d \varphi_{k}\right) u_{n+1, k}+L(\partial) u_{n, k}=0, & \forall n \in \mathbb{N}, k \in\{0,1,4\},\end{cases}
$$

the boundary conditions:

$$
B_{1}\left[\sum_{k \in\{0,1,4\}} u_{n, k}\right]_{\mid x_{1}=0}=\delta_{n, 1} g, \forall n \in \mathbb{N},
$$

and finally the initial conditions:

$$
u_{n, k_{\mid x_{1}=0}}=0, \quad \forall n \in \mathbb{N}, k \in\{0,1,4\} .
$$

The first equation of (5) implies that $u_{0, k} \in \operatorname{ker} \mathscr{L}\left(d \varphi_{k}\right), k \in\{0,1,4\}$ (recall that by definition of $\varphi_{k}$ this kernel is non-trivial). This is the so-called polarization condition.

We introduce $P^{k}$ the projector upon ker $\mathscr{L}\left(d \varphi_{k}\right)$ and $Q^{k}$ the projector on $A_{1} \operatorname{ker} \mathscr{L}\left(d \varphi_{k}\right)$ (we will not give a precise definition of this projectors here, we refer to Definition 25). We have $\operatorname{ker} Q^{k}=\operatorname{Ran} \mathscr{L}\left(d \varphi_{k}\right)$. Thus multiplying the second equation of (5) written for $n=1$ and using the polarization condition $P^{k} u_{0, k}=u_{0, k}$ we obtain:

$$
Q^{k} L(\partial) P^{k} u_{0, k}=0 .
$$

We can then use Lax's lemma [11] which establishs that the previous equation is in fact a transport equation, that is:

$$
\left(\partial_{t}+v_{k} \cdot \nabla_{x}\right) u_{0, k}=0,
$$

where the velocity of the transport $v_{k}$ is the so-called group velocity. Note that the group velocity is by definition (see Definition 5) olinear to the inner normal to $\mathbf{V}$ computed at $\left(\xi_{1}^{k}, \xi_{2}^{k}\right)$ (where $\left(\xi_{1}^{k}, \xi_{2}^{k}\right)$ is the spatial frequency of $\varphi_{k}$ ).

We have easily:

$$
v_{0}:=\left[\begin{array}{c}
-\frac{5}{7} \\
1
\end{array}\right], \quad v_{1}:=\left[\begin{array}{c}
3 \\
-\frac{2}{5}
\end{array}\right], \text { and } v_{4}:=\left[\begin{array}{c}
-\frac{5}{7} \\
1
\end{array}\right],
$$

The interesting point here is that the first component of $v_{1}$ is strictly positive while the second component of each $v_{0}$ and $v_{4}$ are strictly negative. As a consequence, for $k=1$ the transport in (12) goes from the boundary to the interior and it is thus needed to know the value of $u_{0,1}$ at the boundary. But for $k=0,4$ the transport in (12) goes from the interior to the boundary and consequently no boundary condition have to be imposed on the boundary.

From this observation we deduce that for $k=0,4, u_{0, k}$ solves the homogeneous transport equation:

$$
\begin{cases}\left(\partial_{t}+v_{k} \cdot \nabla_{x}\right) u_{0, k}=0, & \text { for } x_{1}>0, x_{2} \in \mathbb{R}, \\ u_{0, k_{\mid t \leq 0}}=0, & \text { for } x_{1}>0, x_{2} \in \mathbb{R},\end{cases}
$$

and we thus have: $u_{0, k} \equiv 0$ for $k=0,4$ and it only remains to determine $u_{0,1}$. To do this we need to know its trace on $\left\{x_{1}=0\right\}$. To determine this trace we use the boundary condition (6) which reads:

$$
B_{1} u_{0,1_{\mid x_{1}=0}}=0 .
$$

However there is an issue. Indeed, from the polarization condition we can write $u_{0,1:=\nu e}$ where $e$ is a generator of $\operatorname{ker} \mathscr{L}\left(1,-\frac{21}{5},-8\right)$. An easy computation gives:

$$
e:=\left[\begin{array}{c}
\frac{7}{3} \\
\sqrt{5} \\
0
\end{array}\right]
$$


which is a transport equation in $\nu$. However to solve (13) we have to make its coefficients more explicit. After some computations we obtain:

$$
P^{1}=\left[\begin{array}{ccc}
\frac{7}{2} & \frac{7}{6} \sqrt{5} & 0 \\
-\frac{3}{2} \sqrt{5} & -\frac{5}{2} & 0 \\
0 & 0 & 0
\end{array}\right], Q^{1}=\left[\begin{array}{ccc}
\frac{7}{2} & -\frac{3}{2} \sqrt{5} & 0 \\
\frac{7}{6} \sqrt{5} & -\frac{5}{2} & 0 \\
0 & 0 & 0
\end{array}\right] \text {, and } R^{1}=\left[\begin{array}{ccc}
\frac{25}{36} & \frac{5}{12} \sqrt{5} & 0 \\
\frac{5}{12} \sqrt{5} & \frac{5}{4} & 0 \\
0 & 0 & -\frac{1}{7}
\end{array}\right] \text {. }
$$

And consequently:

$$
B_{1} R^{1} e=\frac{16}{9} \frac{5}{6} \sqrt{5}, B_{1} R^{1} A_{2} e=\frac{2}{9} \frac{5}{6} \sqrt{5} \text { and } B_{1} R^{1} A_{1} e=0 .
$$

and we remark that $B_{1} e=0$. As a conseuence $\operatorname{ker} \mathscr{L}\left(1,-\frac{21}{5},-8\right) \cap$ ker $B_{1}=$ vect $\{e\}$, the boundary condition satisfied and does not give any information about $u_{0,1_{\left.\right|_{1}=0}}$. It is this situation that we refered happens for a phase with real values we say that we are in the $W R$ class.

To obtain the value of $u_{0,1_{\mid x_{1}=0}}$ we study the only other equation in which $u_{0,1_{\mid x_{1}=0}}$ appears that is the

$$
B_{1} u_{1,1_{\mid x_{1}=0}}=g-B_{1}\left[\sum_{k \in\{0,4\}} u_{1, k}\right]_{\mid x_{1}=0},
$$

in which we make the decomposition $u_{1, k}=P^{k} u_{1, k}+\left(1-P^{k}\right) u_{1, k}$ to obtain:

$$
B_{1} P^{1} u_{1,1_{\mid x_{1}=0}}=g-B_{1}\left[\sum_{k \in\{0,4\}} P^{k} u_{1, k}+\sum_{k \in\{0,4\}}\left(I-P^{k}\right) u_{1, k}\right]_{\mid x_{1}=0}-B_{1}\left(I-P^{1}\right) u_{1,1_{\mid x_{1}=0}},
$$

To make this equation more explicit we first determine the $\left(I-P^{k}\right) u_{1, k}$, that is the unpolarized part. To do this we introduce the pseudo-inverse characterized by:

$$
R^{k} \mathscr{L}\left(d \varphi_{k}\right)=I-P^{k} \text {, and } P^{k} R^{k}=R^{k} Q^{k}=0,
$$

$$
\left(I-P^{k}\right) u_{1, k}=i R^{k} L(\partial) u_{0, k} .
$$

In particular for $k=0,4$ (recall that $u_{0, k} \equiv 0$ ) we obtain that $\left(I-P^{k}\right) u_{1, k} \equiv 0$. To determine $P^{k} u_{1, k}$ we use again Lax's lemma to show that the polarized part satisfies the transport equation:

$$
\begin{cases}\left(\partial_{t}+v_{k} \cdot \nabla_{x}\right) Q^{k} P^{k} u_{1, k}=-Q^{k} L(\partial)\left(I-P^{k}\right) u_{1, k}=0, & \text { for } x_{1}>0, x_{2} \in \mathbb{R} \\ P^{k} u_{1, k_{\mid t \leq 0}}=0, & \text { for } x_{1}>0, x_{2} \in \mathbb{R}\end{cases}
$$

$$
\left(B_{1} R^{1} L(\partial) u_{0,1}\right)_{\mid x_{1}=0}=-i g .
$$

And with the notation $u_{0,1(t, x)=\nu(t, x) e}$ we obtain:

$$
\left(R^{1} e \partial_{t} \nu+R^{1} A_{1} e \partial_{1} \nu+R^{1} A_{2} e \partial_{2} \nu\right)_{\mid x_{1}=0}=-i g,
$$

The transport equation (13) in its final form reads:

$$
\begin{cases}\left(\partial_{t}+\frac{1}{8} \partial_{2}\right) \nu_{\mid x_{1}=0}=-\frac{27}{40 \sqrt{5}} i g, & \text { for }\left(t, x_{2}\right) \in \mathbb{R}^{2}, \\ \nu_{\mid x_{1}=0_{\mid t \leq 0}}=0, & \text { for } x_{2} \in \mathbb{R}\end{cases}
$$


and we can solve this equation by integration along the characteristics to obtain the value of $\nu_{\mid x_{1}=0}$ and then integrate along the characteristics the transport equation for $u_{0,1}$ :

$$
\begin{cases}\left(\partial_{t}+v_{1} \cdot \nabla_{x}\right) u_{0,1}=0, & \text { for }\left(t, x_{1}, x_{2}\right) \in \mathbb{R} \times \mathbb{R}_{+} \times \mathbb{R}, \\ u_{0,1_{\mid x_{1}=0}}=\nu_{\mid x_{1}=0} e, & \text { for }\left(t, x_{2}\right) \in \mathbb{R}^{2}, \\ u_{0,1_{\mid t \leq 0}} & \text { for }\left(x_{1}, x_{2}\right) \in \mathbb{R} \times \mathbb{R}_{+},\end{cases}
$$

to obtain the value of $u_{0,1}$.

An important remark is that from (14), the leading order term $u_{0,1}$ is not zero. But one have to recall that in (3) the source term on the boundary $\left\{x_{1}=0\right\}$ is of order one compared to $\varepsilon$. As a consequence, the leading order of the WKB expansion is of order zero for a source term of order one. It is exactly the amplification phenomenon that we introduced in the introduction. An other remark is that the coefficient in front of the $x_{2}$-derivative in (14) is nothing but $\frac{1}{\xi_{2}^{1}}$.

Then we study the behaviour of the amplitude $u_{0,1}$ as $v_{1}$ as a negative second component, the transported information hits the boundaray $\left\{x_{2}=0\right\}$ after some times. When its happens we formaly consider (recall that $u_{0,1_{\mid x_{2}=0}}$ has its support away from $\left.x_{1}=0\right)$ the boundary value problem in the half space $\left\{x_{1} \in \mathbb{R}, x_{2} \geq 0\right\}$ whose information on the boundary has been turned on by $u_{0,1}$ :

$$
\begin{cases}\partial_{t} u^{\varepsilon}+A_{1} \partial_{1} u^{\varepsilon}+A_{2} \partial_{2} u^{\varepsilon}=0, & \text { for } x_{1} \in \mathbb{R}, x_{2} \in \mathbb{R}_{+}, \\ B_{2} u_{\mid x_{2}=0}^{\varepsilon}=-B_{2} u_{0,1_{\mid x_{2}}=0}, & \text { for } x_{2}=0, x_{1} \in \mathbb{R}, \\ u_{\mid t \leq 0}^{\varepsilon}=0, & \text { for } x_{1} \in \mathbb{R}, x_{2} \in \mathbb{R}_{+},\end{cases}
$$

and we thus add in the ansatz (4) the amplitudes $u_{n, 2}$ associated to the phase $\varphi_{2}$ defined by:

$$
\varphi_{2}(t, x):=t-\frac{21}{5} x_{1}-4 x_{2} .
$$

And the amplitude $u_{n, 5}$ associated to the phase $\varphi_{5}$ defined by:

$$
\varphi_{5}(t, x):=t-\frac{21}{5} x_{1}-\frac{44}{5} x_{2} .
$$

This new phases correspond to the intersection of $\mathbf{V}$ with the line $\left\{\xi_{1}=-\frac{21}{5}\right\}$. Note that the group velocity for the phase $\varphi_{2}$ is given by:

$$
v_{2}:=\left[\begin{array}{c}
-\frac{5}{7} \\
1
\end{array}\right]
$$

while the group velocity $v_{5}$ has both its components positive. From Lax's lemma we have to solve the transport equation:

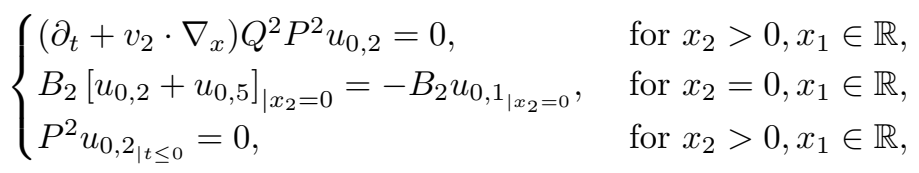

to determine $u_{0,2}$. However, this time we have $\operatorname{ker} \mathscr{L}\left(1,-\frac{21}{5},-4\right)=\operatorname{vect}\left\{e_{2}\right\}$ and $\operatorname{ker} \mathscr{L}\left(1,-\frac{21}{5},-\frac{44}{5}\right)=$ vect $\left\{e_{5}\right\}$ with

$$
e_{2}:=\left[\begin{array}{l}
0 \\
0 \\
1
\end{array}\right], \text { and } e_{5}:=\left[\begin{array}{c}
\sqrt{5} \\
\frac{7}{3} \\
0
\end{array}\right],
$$

and we remark that the matrix $B_{2}$ restricted to vect $\left\{e_{2}, e_{5}\right\}$ is invertible. This is the uniform KreissLopatinskii condition mentioned in the introduction. More precisely this inverse is given by:

$$
\phi^{2}:=\left[\begin{array}{cc}
\frac{3}{7} \sqrt{5} & 0 \\
1 & 0 \\
0 & 1
\end{array}\right]
$$


So we can apply $\phi^{2}$ in the boundary condition and project upon ker $\mathscr{L}\left(1,-\frac{21}{5},-4\right)$ to obtain the value of the trace of $\left.u_{0,2}\right|_{x_{2}=0}$. This permits to solve (17) by integration along the characteristics to determine $u_{0,2}$. The determination of $u_{0,5}$ is similar and will not be describe here.

We now repeat the same process, the group velocity $v_{5}$ has both its components positive, so when one solves the associated transport equation, the transported information spreads to infinity. It will never hits the boundary $\left\{x_{1}=0\right\}$, will never be reflected and consequently it will never creates a new phase by reflection. However the information carried by $u_{0,2}$ will hits the boundary $\left\{x_{1}=0\right\}$ and it will be reflected in a new amplitude. To determine this new amplitude we consider again the boundary value problem in the half space $\left\{x_{1} \geq 0, x_{2} \in \mathbb{R}\right\}$ and we add in the ansatz (4) the amplitudes $u_{n, 3}$ associated to the phase $\varphi_{3}$ defined by:

$$
\varphi_{3}(t, x):=t-3 x_{1}-4 x_{2},
$$

with group velocity:

$$
v_{3}:=\left[\begin{array}{c}
5 \\
-2
\end{array}\right]
$$

$$
\varphi_{4}(t, x):=t-3 x_{1}-8 x_{2}, v_{2}:=\left[\begin{array}{c}
5 \\
-2
\end{array}\right] .
$$

To determine $u_{0,3}$ we have to solve the transport equation:

$$
\begin{cases}\left(\partial_{t}+v_{3} \cdot \nabla_{x}\right) Q^{3} P^{3} u_{0,2}=0, & \text { for } x_{1}>0, x_{2} \in \mathbb{R}, \\ B_{1} u_{0,3}{ }_{\mid x_{1}=0}=-\left.B_{1} u_{0,2}\right|_{x_{1}=0}, & \text { for } x_{1}=0, x_{2} \in \mathbb{R}, \\ P^{3} u_{0,\left.\right|_{\mid t \leq 0}}=0, & \text { for } x_{1}>0, x_{2} \in \mathbb{R},\end{cases}
$$

and we remark that compared to (12), this time $B_{1} e_{3}$, where $e_{3}$ is a generator of ker $\mathscr{L}(1,-3,-4)$ is not zero. As a consequence from the uniform Kreiss-Lopatinskii condition we can invert $B_{1}$ in the boundary condition of (19) to obtain the value of $u_{0,3_{\mid x_{1}=0}}$ and solve (19). An important remark is that this time the amplitude $u_{0,3}$ is not amplified and remains of order $O(1)$ compared with $\varepsilon$.

The information transported by (19) will hit the boundary $\left\{x_{2}=0\right\}$ so it leads us to study the boundary value problem in the half space $\left\{x_{1} \in \mathbb{R}, x_{2} \geq 0\right\}$ whose source term on the boundary has been turned on by the amplitude $u_{0,3}$. So we add in the ansatz the amplitudes for the phases associated to the intersections between $\mathbf{V}$ and the line $\left\{\xi_{1}=-3\right\}$ that is:

$$
\varphi_{6}(t, x):=t-3 x_{1}-\frac{22}{7} x_{2}
$$

and $\varphi_{4}$. It is interesting to remark that even if the amplitude $u_{0,4}$ has been initially removed of the ansatz (because it was initially zero) nothing prevent that this amplitude has to be considered after several rebounds. To dedtermine $u_{0,4}$ we have to solve a transport equation and to do this we need the value of $u_{0,4_{\mid x_{2}=0}}$. Once again we remark that $\operatorname{ker} \mathscr{L}(1,-3,-8)=\operatorname{vect}\left\{e_{4}\right\}$ and $\operatorname{ker} \mathscr{L}\left(1,-3,-\frac{22}{7}\right)=\operatorname{vect}\left\{e_{6}\right\}$, with

$$
e_{4}:=\left[\begin{array}{c}
\sqrt{5} \\
3 \\
0
\end{array}\right], \text { and } e_{6}:=\left[\begin{array}{l}
0 \\
0 \\
1
\end{array}\right]
$$

and consequently by the uniform Kreiss-Lopatinskii condition, the matrix $B_{2}$ restricted to vect $\left.{ }_{4}, e_{6}\right\}$ is invertible. The inverse is given by:

$$
\phi^{4}:=\left[\begin{array}{cc}
\frac{1}{3} \sqrt{5} & 0 \\
1 & 0 \\
0 & 1
\end{array}\right]
$$

which permits to determine the traces $u_{0,4_{\mid x_{2}=0}}$ and to solve the associated transport equation. The transported information will hit the side $\left\{x_{1}=0, x_{2} \in \mathbb{R}\right\}$ and we have to determine its rebounds. To do this we add in the ansatz (4) the phases associated to the intersection point between $\mathbf{V}$ and the line $\left\{\xi_{2}=-8\right\}$. 
It is there interesting to remark that one of these points gives exactly the phase $\varphi_{1}$. As a consequence, the associated amplitude is generated a second time. This phenomenon is refered to selfinteraction phenomenon because it means that some phases can regenerated themselves after a suitable number of rebounds. We thus consider the boundary value problem in the half space $\left\{x_{1} \geq 0, x_{2} \in \mathbb{R}\right\}$ whose source term on the boundary as been turned on by the amplitude $u_{0,4}$ and we add in the ansatz (4) a new contribution for the phase $\varphi_{1}$. The ansatz now reads:

$$
u^{\varepsilon} \sim \sum_{n \geq 0} \sum_{k=1}^{6} e^{\frac{i}{\varepsilon} \varphi_{k}(t, x)} \varepsilon^{n} u_{n, k}+\sum_{n \geq-1} e^{\frac{i}{\varepsilon} \varphi_{1}(t, x)} \varepsilon^{n} \widetilde{u}_{n, 1} .
$$

To determine the new amplitude $\widetilde{u}_{-1,1}$ we again apply Lax's lemma which leads us to solve the transport equation:

$$
\begin{cases}\left(\partial_{t}+v_{1} \cdot \nabla_{x}\right) \widetilde{u}_{0,1}=0, & \text { for } x_{1}>0, x_{2} \in \mathbb{R}, \\ \widetilde{u}_{0,1_{\mid t \leq 0}}=0, & \text { for } x_{1}>0, x_{2} \in \mathbb{R} .\end{cases}
$$

Once again to solve this equation it is needed to determine first the value of the trace $\widetilde{u}_{-1,1_{\mid x_{1}=0}}$. To do this we reiterate the computations made for $u_{0,1}$. That is we consider the equation for $\widetilde{u}_{0,1_{\mid x_{1}=0}}$ We thus obtain that $\widetilde{u}_{-1,1_{\mid x_{1}=0}}$ solves the transport equation (14) but with a source term depending on $u_{0,4_{\mid x_{1}=0}}$ and consequently the amplitude $\widetilde{u}_{-1,1}$ is not zero. So we obtain that the leading order in the WKB expansion is now of order $O\left(\varepsilon^{-1}\right)$ while the source term on the boundary $g^{\varepsilon}$ is of order $O(\varepsilon)$. There are consequently two amplifications in the WKB expansion.

Then we can reiterate exactly the same computations to determine the new amplitudes $\widetilde{u}_{-1, k}, k=2, \ldots, 4$. They do not suffer any amplification due to the Kreiss-Lopatinskii condition, but when one study the rebound of $\widetilde{u}_{-1,4}$. The phase $\varphi_{1}$ is generated again, this induce a new amplification and one has to add in the ansatz a term of order $O\left(\varepsilon^{-2}\right)$ and so on. This process can be repeating an arbitratily number of times to obtain a WKB expansion with $M$ amplifications where $M$ is arbitrarily large.

Of course the previous discussion is totally formal. Indeed, at each step of the computations we restrict ourselves to boundary value problems in the half space and we are not considering boundary value problems in the quarter space as we should. However this discussion has the advantage of simplicity and illustrates the expected phenomenons. The aim of what follows is to give a rigorous justification of this formal analysis.

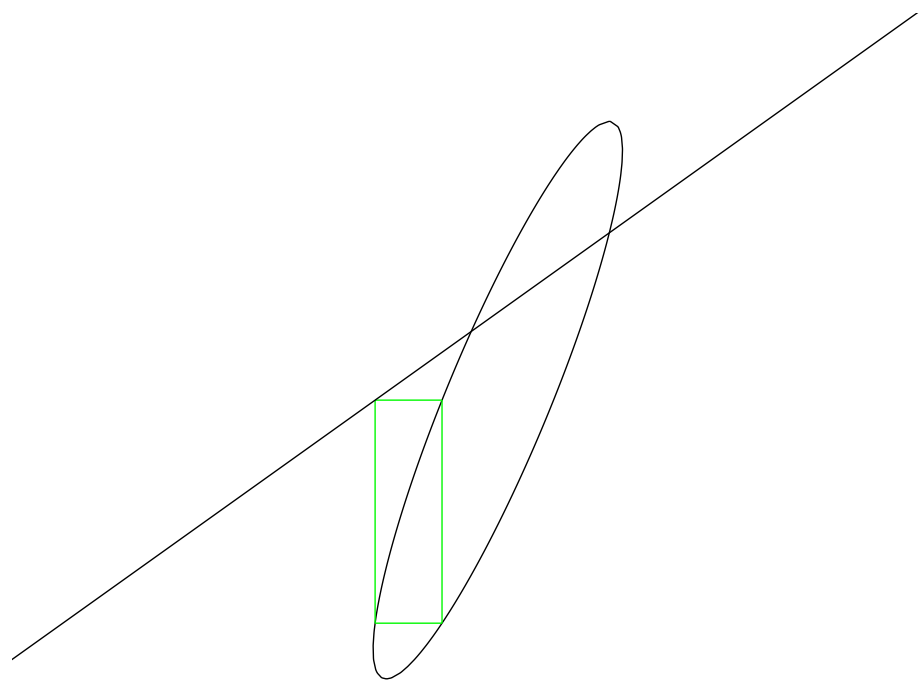

FIG. 1. The characteristic variety for the corner problem (2).

\section{Notations and assumptions.}


For convenience, we also introduce $\Xi_{0}$ the boundary of $\Xi$ :

$$
\Xi_{0}:=\Xi \cap\{\gamma=0\} .
$$

and the notation :

$$
\text { for } j=1,2, \zeta_{j}:=\left(\sigma, \xi_{3-j}\right) \text {, }
$$

\subsection{About the operator $L(\partial)$. Let}

$$
\Omega:=\left\{\left(x_{1}, x_{2}\right) \in \mathbb{R}^{2} \backslash x_{1} \geq 0, x_{2} \geq 0\right\}, \partial \Omega_{1}:=\Omega \cap\left\{x_{1}=0\right\}, \text { and } \partial \Omega_{2}:=\Omega \cap\left\{x_{2}=0\right\},
$$

be the quarter space and both of its edges. For $T>0$, we will denote:

$$
\left.\left.\left.\left.\left.\left.\Omega_{T}:=\right]-\infty, T\right] \times \Omega, \partial \Omega_{1, T}:=\right]-\infty, T\right] \times \partial \Omega_{1}, \text { and } \partial \Omega_{2, T}:=\right]-\infty, T\right] \times \partial \Omega_{2} .
$$

Hereof $\mathscr{L}$ will be the symbol of the differential operator $L(\partial)$. It is defined for $\tau \in \mathbb{R}$ and $\xi \in \mathbb{R}^{2}$ by:

$$
\mathscr{L}(\tau, \xi):=\tau I+\sum_{j=1}^{2} \xi_{j} A_{j}
$$

The characteristic variety $V$ of $L(\partial)$ is thus given by:

$$
V:=\left\{(\tau, \xi) \in \mathbb{R} \times \mathbb{R}^{2} \backslash \operatorname{det} \mathscr{L}(\tau, \xi)=0\right\} .
$$

In this article we choose to restrict our subject, in view to save some notations, to strictly hyperbolic operators. The following constructions of the geometric optics expansions should also operate in the framework of constantly hyperbolic operators. However this simplifying assumption will make the analysis of Section 5 slightly easier. We will give in the core of the proof some comments about the expected modifications about the proof for constantly hyperbolic operators. We thus assume the following property on $L(\partial)$ :

Hypothesis 1 . There exist $N$ real valued functions, denoted by $\lambda_{j}$, analytic on $\mathbb{R}^{2} \backslash\{0\}$ such that:

$$
\forall \xi \in \mathbb{S}^{1}, \operatorname{det} \mathscr{L}(\tau, \xi)=\prod_{j=1}^{N}\left(\tau+\lambda_{j}(\xi)\right),
$$

where the eigenvalues $\lambda_{j}(\xi)$ satisfy $\lambda_{1}(\xi)<\ldots<\lambda_{N}(\xi)$.

We also assume that the boundary of $\Omega$ is non-characteristic, and that the matrices $B_{1}$ and $B_{2}$ induce the

Hypothesis 2. The matrices $A_{1}, A_{2}$ are invertible. Moreover $p_{1}$ (resp. $p_{2}$ ), the number of lines of $B_{1}$ (resp. $B_{2}$ ), equals the number of strictly positive eigenvalues of $A_{1}$ (resp. $A_{2}$ ). At last $B_{1}$ and $B_{2}$ are assumed to be of maximal rank.

One can easily convince himself that they are effectively the good number of conditions by considering the

Under Hypothesis 2, we can define the resolvent matrices with are obtained by taking the Laplace transform of the evolution equation of (1) and Fourier transform in one of the space variable:

$$
\mathscr{A}_{1}(\zeta):=-A_{1}^{-1}\left(\sigma I+i \eta A_{2}\right) \text { and } \mathscr{A}_{2}(\zeta):=-A_{2}^{-1}\left(\sigma I+i \eta A_{1}\right),
$$

where $\zeta$ denotes an element of the frequency space :

$$
\Xi:=\{\zeta:=(\sigma=\gamma+i \tau, \eta) \in \mathbb{C} \times \mathbb{R}, \gamma \geq 0\} \backslash\{(0,0)\} .
$$

For $j=1,2, \zeta_{j} \in\left(\Xi \backslash \Xi_{0}\right)$, we denote by $E_{j}^{s}\left(\zeta_{j}\right)$ the stable subspace of $\mathscr{A}_{j}\left(\zeta_{j}\right)$ and $E_{j}^{u}\left(\zeta_{j}\right)$ its unstable subspace. These spaces are well-defined according to [9]. For all $\zeta_{j} \in\left(\Xi \backslash \Xi_{0}\right)$, the stable subspace $E_{j}^{s}\left(\zeta_{j}\right)$ has dimension $p_{j}$, while the space $E_{j}^{u}(\zeta)$ has dimension $N-p_{j}$. Let us recall the following theorem due to Kreiss $[10]^{2}$ :

\footnotetext{
${ }^{2}$ For constantly (resp. geometrically regular) hyperbolic operators, this Theorem still holds, see [15] (resp. [16]).
} 
TheOREm 3. Under Hypothesis 1 and 2 , for all $\zeta \in \Xi$, there exist a neighborhood $\mathscr{V}$ of $\zeta$ in $\Xi$, integers $L_{1}, L_{2} \geq 1$, two partitions $N=\nu_{1,1}+\ldots+\nu_{1, L_{1}}=\nu_{2,1}+\ldots+\nu_{2, L_{2}}$ with $\nu_{1, l}, \nu_{2, l} \geq 1$, and two invertible matrices $T_{1}, T_{2}$, regular on $\mathscr{V}$ such that:

$$
\begin{aligned}
\forall \zeta \in \mathscr{V}, & T_{1}(\zeta)^{-1} \mathscr{A}_{1}(\zeta) T_{1}(\zeta)=\operatorname{diag}\left(\mathscr{A}_{1,1}(\zeta), \ldots, \mathscr{A}_{1, L_{1}}(\zeta)\right), \\
& T_{2}(\zeta)^{-1} \mathscr{A}_{2}(\zeta) T_{2}(\zeta)=\operatorname{diag}\left(\mathscr{A}_{2,1}(\zeta), \ldots, \mathscr{A}_{2, L_{2}}(\zeta)\right),
\end{aligned}
$$

where the blocks $\mathscr{A}_{j, l}(\zeta)$ have size $\nu_{j, l}$ and satisfy one of the following alternatives:

i) All the elements in the spectrum of $\mathscr{A}_{j, l}(\zeta)$ have positive real part.

ii) All the elements in the spectrum of $\mathscr{A}_{j, l}(\zeta)$ have negative real part.

iii) $\nu_{j, l}=1, \mathscr{A}_{j, l}(\zeta) \in i \mathbb{R}, \partial_{\gamma} \mathscr{A}_{j, l}(\zeta) \in \mathbb{R} \backslash\{\overline{0}\}$, and $\mathscr{A}_{j, l}(\zeta) \in i \mathbb{R}$ for all $\zeta \in \mathscr{V} \cap \Xi_{0}$.

iv) $\nu_{j, l}>1, \exists k_{j, l} \in i \mathbb{R}$ such that

$$
\mathscr{A}_{j, l}(\underline{\zeta})=\left[\begin{array}{ccc}
k_{j, l} & i & 0 \\
& \ddots & i \\
0 & & k_{j, l}
\end{array}\right],
$$

the coefficient in the lower left corner of $\partial_{\gamma} \mathscr{A}_{j, l}(\zeta)$ is real and non-zero, and moreover $\mathscr{A}_{j, l}(\zeta) \in i \boldsymbol{M}_{\nu_{j, l}}(\mathbb{R})$ for all $\zeta \in \mathscr{V} \cap \Xi_{0}$.

Thanks to Theorem 3 it is possible to describe the four kinds of frequencies, for each part of the boundary $\partial \Omega$ :

Definition 4. For $j=1,2$, we denote by:

1) $\mathscr{E}_{j}$ the set of elliptic frequencies, that is to say the set of $\zeta \in \Xi_{0}$ such that Theorem 3 for the matrix $\mathscr{A}_{j}(\zeta)$ is satisfied with one block of type $i$ ) and one block of type $\overline{i i}$ ) only.

2) $\mathscr{H}_{j}$ the set of hyperbolic frequencies, that is to say the set of $\zeta \in \Xi_{0}$ such that Theorem 3 for the matrix $\mathscr{A}_{j}(\zeta)$ is satisfied with blocks of type iii) only.

3) $\mathscr{E} \mathscr{H}_{j}$ the set of mixed frequencies, that is to say the set of $\zeta \in \Xi_{0}$ such that Theorem 3 for the matrix $\mathscr{A}_{j}(\underline{\zeta})$ is satisfied with one block of type $\left.i\right)$, one of type ii) and at least one of type iii), but without block of type iv).

4) $\mathscr{G}_{j}$ the set of glancing frequencies, that is to say the set of $\zeta \in \Xi_{0}$ such that Theorem 3 for the matrix $\mathscr{A}_{j}(\zeta)$ is satisfied with at least one block of type iv).

Thus, by definition, $\Xi_{0}$ admits the following decomposition:

$$
\Xi_{0}=\mathscr{E}_{j} \cup \mathscr{E} \mathscr{H}_{j} \cup \mathscr{H}_{j} \cup \mathscr{G}_{j} .
$$

The study made in [10] shows that the subspaces $E_{1}^{s}\left(\zeta_{j}\right)$ and $E_{2}^{s}\left(\zeta_{j}\right)$ admit a continuous extension up to the boundary of the frequency set $\Xi_{0}$. Moreover, for $j=1,2$, for $\underline{\zeta}_{j} \in \Xi_{0} \backslash\left(\mathscr{G}_{1} \cup \mathscr{G}_{2}\right)$ one can decompose:

$$
\mathbb{C}^{N}=E_{j}^{s}\left(i \underline{\tau}, \underline{\xi}_{3-j}\right) \oplus E_{j}^{u}\left(i \underline{\tau}, \underline{\xi}_{3-j}\right),
$$

where the spaces $E_{j}^{s}$ and $E_{j}^{u}$ can also be decomposed in the following way:

$$
E_{j}^{s}\left(i \underline{\tau}, \underline{\xi}_{3-j}\right)=E_{j}^{s, e}\left(i \underline{\tau}, \underline{\xi}_{3-j}\right) \oplus E_{j}^{s, h}\left(i \underline{\tau}, \underline{\xi}_{3-j}\right) \text {, and } E_{j}^{u}\left(i \underline{\tau}, \underline{\xi}_{3-j}\right)=E_{j}^{u, e}\left(i \underline{\tau}, \underline{\xi}_{3-j}\right) \oplus E_{j}^{u, h}\left(i \underline{\tau}, \underline{\xi}_{3-j}\right) .
$$

Here $E_{j}^{s, e}\left(i \underline{\tau}, \underline{\xi}_{3-j}\right)\left(\operatorname{resp} . E_{j}^{u, e}\left(i \underline{\tau}, \underline{\xi}_{3-j}\right)\right)$ is the generalized eigenspace associated with eigenvalues of the matrix $\mathscr{A}_{j}\left(i \underline{\tau}, \underline{\xi}_{3-j}\right)$ with negative (resp. positive) real part, and where spaces $E_{j}^{s, h}\left(i \underline{\tau}, \underline{\xi}_{3-j}\right)$ and $E_{j}^{u, h}\left(i \underline{\tau}, \underline{\xi}_{3-j}\right)$ are sums of eigenspaces of $\mathscr{A}_{j}\left(i \underline{\tau}, \underline{\xi}_{3-j}\right)$ associated with some purely imaginary eigenvalues of $\mathscr{A}_{j}\left(i \underline{\tau}, \underline{\xi}_{3-j}\right)$.

Moreover since the matrices $A_{1}$ and $A_{2}$ are invertible we can also write (27) in the following way :for $j=1,2$

$$
\mathbb{C}^{N}=A_{j} E_{j}^{s}\left(i \underline{\tau}, \underline{\xi}_{3-j}\right) \oplus A_{j} E_{j}^{u}\left(i \underline{\tau}, \underline{\xi}_{3-j}\right),
$$

In fact, it is possible to give a more precise decomposition of the spaces $E_{j}^{s, h}\left(\underline{\zeta}_{j}\right)$ and $E_{j}^{u, h}\left(\underline{\zeta}_{j}\right)$. Indeed, let $\underline{\omega}_{m, j}$ be a purely imaginary eigenvalue of $\mathscr{A}_{j}\left(\underline{\zeta}_{j}\right)$, that is satisfying:

$$
\operatorname{det}\left(\underline{\tau}+\underline{\eta}_{1}+\underline{\omega}_{m, 2} A_{2}\right)=\operatorname{det}\left(\underline{\tau}+\underline{\omega}_{m, 1} A_{1}+\underline{\eta} A_{2}\right)=0 .
$$


Then, using Hypothesis 1, we deduce that there exists an index $k_{m, j}$ satisfying:

$$
\underline{\tau}+\lambda_{k_{m, 2}}\left(\underline{\eta}^{\prime} \underline{\omega}_{m, 2}\right)=\underline{\tau}+\lambda_{k_{m, 1}}\left(\underline{\omega}_{m, 1}, \underline{\eta}\right)=0,
$$

where $\lambda_{k_{m, j}}$ is smooth in both variables. Let us then introduce the following classification :

DEFINITION 5. - The set of incoming (resp. outgoing) phases for the side $\partial \Omega_{1}$, denoted by $\mathfrak{I}_{1}$ (resp. $\left.\mathfrak{O}_{1}\right)$, is the set of indices $m$ such that the group velocity $v_{m}:=\nabla \lambda_{k_{m, 1}}\left(\underline{\omega}_{m, 1}, \underline{\eta}\right)$ satisfies $\partial_{1} \lambda_{k_{m, 1}}\left(\underline{\omega}_{m, 1}, \underline{\eta}\right)>0$ (resp. $\left.\partial_{1} \lambda_{k_{m, 1}}\left(\underline{\omega}_{m, 1}, \underline{\eta}\right)<0\right)$.

- The set of incoming (resp. outgoing) phases for the side $\partial \Omega_{2}$, denoted by $\mathfrak{I}_{2}$ (resp. $\mathfrak{O}_{2}$, is the set of indices $m$ such that the group velocity $v_{m}:=\nabla \lambda_{k_{m, 2}}\left(\eta, \underline{\omega}_{m, 2}\right)$ satisfies $\partial_{2} \lambda_{k_{m, 2}}\left(\eta, \underline{\omega}_{m, 2}\right)>0$ (resp. $\left.\partial_{2} \lambda_{k_{m, 2}}\left(\eta, \underline{\omega}_{m, 2}\right)<0\right)$.

- The set of incoming-incoming (resp. outgoing-outgoing) phases $\mathscr{I}_{i i}$ (resp. $\mathscr{I}_{\text {oo }}$ ) is defined by $I_{i i}:=$ $\mathfrak{I}_{1} \cap \mathfrak{I}_{2}$ (resp. $\left.\mathscr{I}_{\text {oo }}:=\mathfrak{O}_{1} \cap \mathfrak{O}_{2}\right)$.

- The set of incoming-outgoing (resp. outgoing-incoming) phases $\mathscr{I}_{\text {io }}$ (resp. $\mathscr{I}_{\text {oi }}$ ) is defined by $\mathscr{I}_{i o}:=$ $\mathfrak{I}_{1} \cap \mathfrak{O}_{2}$ (resp. $\left.\mathscr{I}_{o i}:=\mathfrak{O}_{1} \cap \mathfrak{I}_{2}\right)$.

Note that we do not considered the phases whose one of the component of the group velocity vanishs. This phases correspond to glancing modes and the construction of the geometric optics expansions is much more technical when we consider these phases [20].

Thanks to Definition 5, we can write the following decomposition of the stable and unstable components $E_{j}^{s, h}(\underline{\zeta})$ and $E_{j}^{u, h}(\underline{\zeta})$ :

LEMma 6. For all $\underline{\zeta} \in \mathscr{H}_{j} \cup \mathscr{E} \mathscr{H}_{j}, j=1,2$ we have

$$
\begin{array}{ll}
E_{1}^{s, h}(\underline{\zeta})=\oplus_{m \in \mathfrak{I}_{1}} \operatorname{ker} \mathscr{L}\left(\underline{\tau}, \underline{\omega}_{m, 1}, \underline{\eta}\right), & E_{1}^{u, h}(\underline{\zeta})=\oplus_{m \in \mathfrak{O}_{1}} \operatorname{ker} \mathscr{L}\left(\underline{\tau}, \underline{\omega}_{m, 1}, \underline{\eta}\right), \\
E_{2}^{s, h}(\underline{\zeta})=\oplus_{m \in \mathfrak{I}_{2}} \operatorname{ker} \mathscr{L}\left(\underline{\tau}, \underline{\eta}, \underline{\omega}_{m, 2}\right), & E_{2}^{u, h}(\underline{\zeta})=\oplus_{m \in \mathfrak{O}_{2}} \operatorname{ker} \mathscr{L}\left(\underline{\tau}, \underline{\eta}, \underline{\omega}_{m, 2}\right) .
\end{array}
$$

We refer, for example, to [8] or [20] for a proof of this lemma.

3.2. About the boundary conditions. Let us introduce the initial boundary value problem in the half space $\left\{x_{1} \geq 0, x_{2} \in \mathbb{R}\right\}$ :

$$
\left\{\begin{array}{l}
L(\partial) u=f, \text { on }\left\{x_{1} \geq 0, x_{2} \in \mathbb{R}\right\} \\
B_{1} u_{\mid x_{1}=0}=g_{1}, \\
u_{\mid t \leq 0}=0 .
\end{array}\right.
$$

We recall the following result due to Kreiss [10] which establishs that the boundary value problem (32) is strongly well-posed if and only if it satisfies the following condition:

DEFINITION 7 (Uniform Kreiss-Lopatinskii condition). We say that the system (32) satisfies the uniform Kreiss-Lopatinskii condition if we have:

$$
\forall \zeta \in \Xi, \operatorname{ker} B_{1} \cap E_{1}^{s}(\zeta)=\{0\}
$$

where $E_{1}^{s}(\zeta)$ still denotes the continuation of the stable subspace of the resolvent matrix $\mathscr{A}_{1}(\zeta)$ up to $\Xi_{0}$.

DEFINITION 8. We denote by $\Upsilon$ the set of frequencies for which the ibvp (32) does not satisfy the uniform Kreiss-Lopatinskii condition i.e.

$$
\Upsilon:=\left\{\zeta_{1} \in \Xi: \operatorname{ker} B_{1} \cap E_{1}^{s}\left(\zeta_{1}\right) \neq\{0\}\right\} .
$$

If $\Upsilon \cap\left(\Xi \backslash \Xi_{0}\right) \neq \emptyset$ then we say that this problem does not satisfy the Kreiss-Lopatinskii condition and it is a well-known fact, we refer for example to [10] or [6], that such problem can not be strongly or weakly well-posed. As a consequence, the only possible frequencies where the uniform Kreiss-Lopatinskii can fail without causing ill-posedness of the problem are located on $\Xi_{0}$.

The kind of degeneracy that we will consider in this article are thus on $\Xi_{0}$ and correspond to hyperbolic frequencies that is that $\mathscr{A}_{1}(\underline{\zeta})$ is diagonalizable with only imaginary eigenvalues. We recall that these frequencies induce a transport of the information. More precisely we will use the following definition due to [5]: 
DeFINITION 9. The ibvp (32) is in the $W R$ class if it satisfies the following conditions:

i) The ibvp (32) satisfies the weak Kreiss-Lopatinskii condition i.e. $\Upsilon \cap\left(\Xi \backslash \Xi_{0}\right)=\emptyset$.

ii) $\Upsilon \neq \emptyset$ and $\Upsilon \subset \mathscr{\mathscr { H }}_{1}$.

iii) For all $\underline{\zeta} \in \Upsilon$, there is a neighborhood $\mathscr{V}$ of $\zeta$ in $\Xi$, a regular basis $\left(E_{1,1}^{s}, \ldots, E_{1, p_{1}}^{s}\right)(\zeta)$ of $E_{1}^{s}(\zeta)$ on $\mathscr{V}$, an invertible $p_{1} \times p_{1}$ matrix $P(\zeta)$, regular on $\mathscr{V}$ and a smooth real valued function $\Theta$ such that

$$
\forall \zeta \in \mathscr{V}, B_{1}\left[E_{1,1}^{s}, \ldots, E_{1, p_{1}}^{s}\right](\zeta)=P(\zeta) \operatorname{diag}(\gamma+i \Theta(\zeta), 1, \ldots, 1) .
$$

In particular, one can find a Lopatinskii's determinant under the form:

$$
\forall \zeta \in \mathscr{V}, \Delta(\zeta)=(\gamma+i \Theta(\zeta)) \operatorname{det} P(\zeta)
$$

These definitions about hyperbolic boundary value problems in the half space motivate the following definition for the corner problem:

DEFINITION 10. We say that the corner problem (1) is in the $W R$ class for the side $\partial \Omega_{1}$ if the boundary value problem (32) is in the $W R$ class.

In all this paper, the boundary condition for the side $\partial \Omega_{1}$ will not satisfy the uniform Kreiss-Lopatinskii condition (precisely we will then work with corner problem in the $W R$ class for the side $\partial \Omega_{1}$ ) and so weak well-posedness is expected. While, as mentioned in the introduction, the boundary condition for the side $\partial \Omega_{2}$ will be chosen as convenient as possible in terms of well-posedness. However, we will see in Theorem 14 that even if the boundary condition on $\partial \Omega_{2}$ is in the most favorable class of strictly dissipative boundary conditions (see [6]), it can not compensate the "bad" boundary condition on $\partial \Omega_{1}$.

To make things more precise, let us thus assume the following :

Hypothesis 11. The corner problem (1) is in the $W R$ class for the side $\partial \Omega_{1}$ and the boundary condition on the side $\partial \Omega_{2}$ is strictly dissipative or satisfies (at least) the uniform Kreiss-Lopatinskii condition. In the first case, that is to say that the following inequality holds:

$$
\forall v \in \operatorname{ker} B_{2},\left\langle A_{2} v, v\right\rangle<0 \text {. }
$$

While in the second case, it means that $\forall \zeta \in \Xi$, we have:

$$
\text { ker } B_{2} \cap E_{2}^{s}(\zeta)=\{0\} .
$$

In both cases (we refer to [6] for a proof that strict dissipativity implies the uniform Kreiss-Lopatinskii condition) the restriction of $B_{2}$ to $E_{2}^{s}(\zeta)$ is invertible. We denote this inverse by $\phi_{2}(\zeta)$.

When one studies geometric optics expansion for weakly well-posed boundary value problems, it is useful to define the following vectors (this definition comes from [8] and was also used later in [2]) :

DeFINITION 12. Let (1) be in the WR class for the side $\partial \Omega_{1}$, then there exist:

$\diamond$ a vector $e \in \mathbb{C}^{N} \backslash\{0\}$ such that $\operatorname{ker} B_{1} \cap E_{1}^{s}(\zeta)=\operatorname{vect}(e)$.

$\diamond A$ vector $b \in \mathbb{C}^{p_{1}} \backslash\{0\}$ such that $b \cdot B w=0$, for all $w \in E_{1}^{s}(\zeta)$.

4. Formal phase generation process. In this paragraph we briefly discuss the expected phases in the WKB expansion and the associated amplitudes for corner problem in the $W R$ class for the side $\partial \Omega_{1}$. We will not here give a precise description of the phase generation process in itself (we refer to [4] and [19] for a complete discussion) but we will focus on the expected sizes of the amplitudes according to the small parameter $\varepsilon$. More especially we will also discuss after how many time of travel the amplifications are expected.

Let us consider the hyperbolic corner problem:

$$
\left\{\begin{array}{l}
L(\partial) u^{\varepsilon}=0, \text { on } \Omega_{T} \\
B_{1} u_{\mid x_{1}=0}^{\varepsilon}=g^{\varepsilon}, \text { on } \partial \Omega_{1, T}, \\
B_{2} u_{\mid x_{2}=0}^{\varepsilon}=0, \text { on } \partial \Omega_{2, T}, \\
u_{\mid t \leq 0}^{\varepsilon}=0,
\end{array}\right.
$$

and we assume that (35) satisfies Assumptions 1-2 and 11. We also suppose that this corner problem admits four selfinteracting planar phases (see Assumption 21 or [4] for more details) namely

$$
\varphi_{n_{j}}(t, x):=\underline{\tau} t+\xi_{1}^{n_{j}} x_{1}+\xi_{2}^{n_{j}} x_{2}, j=1, \ldots 4 .
$$


To be considered as selfinteracting these phases have firstly to satisfy

$$
\xi_{1}^{n_{1}}=\xi_{1}^{n_{2}}, \xi_{2}^{n_{2}}=\xi_{2}^{n_{3}}, \xi_{1}^{n_{3}}=\xi_{1}^{n_{4}}, \text { and } \xi_{2}^{n_{4}}=\xi_{2}^{n_{1}},
$$

The ray associated to the phase $\varphi_{n_{1}}$ is incoming-outgoing so it hits the side $\partial \Omega_{2}$ after a finite time of
el. However, when it happens, the striking ray still have its support away from the corner. Thus, once
, by finite speed of propagation arguments, at least during a small time, we can see the corner problem
as a problem in the half space $\left\{x_{1} \in \mathbb{R}, x_{2}>0\right\}$ for which the information on the side $\partial \Omega_{2}$ has been

The ray associated to the phase $\varphi_{n_{1}}$ is incoming-outgoing so it hits the side $\partial \Omega_{2}$ after a finite time of
travel. However, when it happens, the striking ray still have its support away from the corner. Thus, once
again, by finite speed of propagation arguments, at least during a small time, we can see the corner problem
(35) as a problem in the half space $\left\{x_{1} \in \mathbb{R}, x_{2}>0\right\}$ for which the information on the side $\partial \Omega_{2}$ has been

The ray associated to the phase $\varphi_{n_{1}}$ is incoming-outgoing so it hits the side $\partial \Omega_{2}$ after a finite time of
travel. However, when it happens, the striking ray still have its support away from the corner. Thus, once
again, by finite speed of propagation arguments, at least during a small time, we can see the corner problem
(35) as a problem in the half space $\left\{x_{1} \in \mathbb{R}, x_{2}>0\right\}$ for which the information on the side $\partial \Omega_{2}$ has been

The ray associated to the phase $\varphi_{n_{1}}$ is incoming-outgoing so it hits the side $\partial \Omega_{2}$ after a finite time of
travel. However, when it happens, the striking ray still have its support away from the corner. Thus, once
again, by finite speed of propagation arguments, at least during a small time, we can see the corner problem
(35) as a problem in the half space $\left\{x_{1} \in \mathbb{R}, x_{2}>0\right\}$ for which the information on the side $\partial \Omega_{2}$ has been

in other words it is possible to draw a rectangle whose vertices are on the characteristic variety. We will also impose that the phase $\varphi_{n_{1}}$ and $\varphi_{n_{3}}$ are incoming-outgoing whereas $\varphi_{n_{2}}$ and $\varphi_{n_{4}}$ are outgoing-incoming in view to have the good velocities for a selfinteraction.

We also choose the source term $g^{\varepsilon}$ in (35) in such a way that it "turns on" the phase $\varphi_{n_{1}}$. Finally we assume that the phase $\varphi_{n_{1}}$ is associated to the only frequency for which the uniform Kreiss-Lopatinskii condition is violated.

Let us choose $g^{\varepsilon}$, zero for negative times, with compact support away from the corner for all positive times (or at least a function which is zero on a neighborhood of the corner for all positive times). Then by finite speed of propagation arguments, the information carried by $g^{\varepsilon}$ can not hit the side $\partial \Omega_{2}$ immediately. So, at least during a short time, we can forget the boundary condition on $\left\{x_{1}>0, x_{2}=0\right\}$ and see the corner problem (35) as a problem in the $W R$ class for the half space $\left\{x_{1}>0, x_{2} \in \mathbb{R}\right\}$. It is thus natural to start by taking the ansatz for this problem lying in the half space. This ansatz involves the phases $\varphi_{j}$ defined by:

$$
\varphi_{j}(t, x):=\underline{\tau} t+\xi_{1}^{j} x_{1}+\xi_{2}^{n_{1}} x_{2}, j=1, \ldots N,
$$

where the $\left(\xi_{1}^{j}\right)$ are the roots in the $\xi_{1}$ variable of the dispersion relation:

$$
\operatorname{det} \mathscr{L}\left(\underline{\tau}, \xi_{1}, \xi_{2}^{n_{1}}\right)=0,
$$

associated to incoming-outgoing or incoming-incoming group velocities (see [4] for more details). Let us stress that, by assumption, the phase $\varphi_{n_{1}}$ is contained in the $\left\{\varphi_{j}\right\}_{j}$ and that since the corner problem (35) is in the WR class, in particular $\left(i \underline{\tau}, \xi_{2}^{n_{1}}\right) \in \mathscr{H}_{1}$, then there is exactly $N$ roots in the $\xi_{1}$ variable of the dispersion relation $\operatorname{det} \mathscr{L}\left(\underline{\tau}, \xi_{1}, \xi_{2}^{n_{1}}\right)=0$.

However the analysis of [8] tells us that when the uniform Kreiss-Lopatinskii condition breaks down, due to a transport phenomenon along the side $\partial \Omega_{1}$, the leading order is one order less than the order of the source term on the boundary $\left\{x_{1}=0, x_{2}>0\right\}$. More precisely, if the source term is of order one, then the leading order in the WKB expansion is of order zero. This transport phenomenon along the side $\partial \Omega_{1}$, in the presence of a source term on the boundary, is immediately turned on. We thus expect that this property which comes from the study of the problem in the half space remains true for the corner problem (35), at least during a short time.

In all this paper, we will assume that the transport along the side $\partial \Omega_{1}$ spreads the information away from the corner. If it is not the case then the information will hit the corner in finite time. Until now, we are not able to construct geometric optics expansions if such a situation occurs and we thus have a maximal time of existence for the geometric optics expansions corresponding to the time of impact. However as first noticed in [8], in the particular framework $d=2$, then the velocity of the transport along the side $\partial \Omega_{1}$ is explicitly computable. More precisely it is given by $-\frac{\tau}{\xi_{2}^{n_{1}}}$ (see [8]). So in this particular setting ask that the transport phenomenon along the side $\partial \Omega_{1}$ spreads the information away from the corner is equivalent to ask that $\underline{\tau}$ and $\xi_{2}^{n_{1}}$ have opposite signs, which is easily verifiable in practice.

The rays associated to the phase $\varphi_{j}$ are incoming-outgoing or incoming-incoming. As a consequence if the phase $\varphi_{j}$ the information that it carried will hit the boundary $\partial \Omega_{2}$ after some time of travel and we will have to determine its reflections or if it is incoming-incoming then it escapes to infinity and it will never be reflected. Let us now study the reflections of the incoming-outgoing ones. More precisely we will only describe the reflections $\varphi_{n_{1}}$ which are the capital ones for our current discussion. We refer to [4] for a precise description of the reflections for the other rays. 
"turned on" by the incoming-outgoing phase $\varphi_{n_{1}}$. We thus add in the ansatz the amplitudes associated with the phases

$$
\varphi_{j}(t, x):=\underline{\tau} t+\xi_{1}^{n_{1}} x_{1}+\xi_{2}^{j} x_{2}, j=1, \ldots N_{2},
$$

where the $\left(\xi_{2}^{j}\right)$ are the roots in the $\xi_{2}$ variable of the dispersion relation :

$$
\operatorname{det} \mathscr{L}\left(\underline{\tau}, \xi_{1}^{n_{1}}, \xi_{2}\right)=0
$$

Note that $\varphi_{n_{2}}$ is included in this set.

Let us here insist on the fact that there can be less than $N$ roots for this dispersion relation and that these roots can be complex valued. Indeed, the boundary value problem in the half space $\left\{x_{1} \in \mathbb{R}, x_{2}>0\right\}$ is not assumed to be in the $W R$ class. According to [4] we only consider the real roots associated with outgoing-incoming or incoming-incoming group velocities and a class representative of the complex valued roots with positive imaginary part (see Section 6 or [4] for more details).

As the boundary condition on the side $\partial \Omega_{2}$ is assumed to satisfy the uniform Kreiss-Lopatinskii condition, this time there is no transport phenomenon along the boundary and the leading order of the $W K B$ expansion remains of order zero (for a source term on the boundary $\partial \Omega_{1}$ of order one).

We can then repeat exactly the same arguments to show that the phases $\varphi_{n_{3}}$ and $\varphi_{n_{4}}$ are generated when we consider the reflections of the ray associated to $\varphi_{n_{2}}$ on $\partial \Omega_{1}{ }^{3}$ and the reflection of the ray associated to $\varphi_{n_{3}}$ on $\partial \Omega_{2}$.

It is then interesting to study the reflection of the ray associated to $\varphi_{n_{4}}$ against the side $\partial \Omega_{1}$. This reflection starts after a strictly positive time $t_{0}$ at a strictly positive distance of the corner $y_{0}$ (the precise values of this parameters are given in Section 7 and will be fundamental in our proof). By selfinteraction phenomenon, we know that during this reflection the phase $\varphi_{n_{1}}$ is regenerated. However due to the degeneracy of the uniform Kreiss-Lopatinskii condition for the phase $\varphi_{n_{1}}$, a new transport along the boundary is expected and shall induce a new amplification phenomenon. All the amplitudes in the ansatz then should lose one power of $\varepsilon$, that is to say that the amplitude of order zero becomes of order -1 , the amplitude of order one becomes of order zero and so on.

As a consequence, if one starts with a source term of order two in $\varepsilon$ then before the time $t_{0}$, the leading order of the geometric optics expansion is expected to be of order one, but after the time $t_{0}$, the leading order in this expansion is expected to be of order zero. Moreover the traces of the amplitudes associated to the phase $\varphi_{n_{1}}$ are now expected to be zero for $x_{2}$ less than $y_{0}$. We can then repeat exactly the same arguments. That is to say that after another complete circuit around the loop, the amplitude associated to $\varphi_{n_{4}}$ hits again the side $\partial \Omega_{1}$ and regenerates the phase $\varphi_{n_{1}}$. A new amplification thus happens and all the amplitudes lose one order in terms of $\varepsilon$. The leading order in the geometric optics expansion is now expected to be of order zero for a source term on the boundary of order three.

So if one wants to construct a geometric optics expansion with $M+1$ amplifications before a fixed time $T$, it will be sufficient to choose the support of the source term $g$ close enough of the corner to ensure that the rays have made $M$ complete circuits around the loop before the time $T$.

Moreover, the fact that we choose to work with a fixed maximal time of resolution $T$ and with a source term $g$ having its support away from the corner permits us to assume that the number of generated phases in the phase generation process is finite and avoid the technical difficulties pointed in [4]. Indeed, in [4] it was shown that the phase generation process consists of considering sequences of phases with incoming-outgoing and then outgoing-incoming group velocities and to stop the sequence when we meet a phase with incomingincoming group velocity. However, here each transport phenomenon from one side to the other takes some (explicitly computable) time) because the transported information has its support away from the corner. So when we apply the phase generation process we can stop the sequence as soon as the sum of the time needed to generate the considered phase exceeds the fixed maximal time $T$.

5. Main result. In this paragraph we describe the main result of this paper. Roughly speaking, this result states that when one deals with a corner problem that does not satisfy the uniform Kreiss-Lopatinskii

\footnotetext{
${ }^{3}$ Let us stress that during this reflection on the side $\partial \Omega_{1}$ there is no amplification because the considered phases are $\varphi_{n_{2}}$ and $\varphi_{n_{3}}$ but not $\varphi_{n_{1}}$.
} 
condition on each boundary then he should be careful. Indeed, Theorem 14 demonstrates that such a problem can, in some situations depending on the geometry of the characteristic variety, be ill-posed. The violent instability is, in this framework, caused by an accumulation of weak instabilities and differs from the failure of the weak Kreiss-Lopatinskii condition which is, to our knowledge, the only known example of violent instability for hyperbolic boundary value problem. Before to state the main theorem, let us define more precisely the terms of the previous discussion:

DEFINITION 13. The corner problem (1) is said to be weakly well-posed (or to generate a weak instability) if there exists $\left(K, K_{1}, K_{2}\right) \in \mathbb{R}_{+}^{3} \backslash\{0\}$ such that for all source terms $f \in H^{K}\left(\Omega_{T}\right), g_{1} \in H^{K_{1}}\left(\partial \Omega_{1, T}\right), g_{2} \in$ $H^{K_{2}}\left(\partial \Omega_{2, T}\right)$, the corner problem (1) admits a unique solution $u \in L^{2}\left(\Omega_{T}\right)$ with traces $u_{\mid x_{1}=0} \in L^{2}\left(\partial \Omega_{1, T}\right)$, $u_{\mid x_{2}=0} \in L^{2}\left(\partial \Omega_{2, T}\right)$ satisfying the energy estimate:

$$
\begin{gathered}
\|u\|_{L^{2}\left(\Omega_{T}\right)}^{2}+\left\|u_{\mid x_{1}=0}\right\|_{L^{2}\left(\partial \Omega_{1, T}\right)}^{2}+\left\|u_{\mid x_{2}=0}\right\|_{L^{2}\left(\partial \Omega_{2, T}\right)}^{2} \leq \\
C_{T}\left(\|f\|_{H^{K}\left(\Omega_{T}\right)}^{2}+\left\|g_{1}\right\|_{H^{K_{1}}\left(\partial \Omega_{1, T}\right)}^{2}+\left\|g_{2}\right\|_{H^{K_{2}\left(\partial \Omega_{2, T}\right)}}^{2}\right),
\end{gathered}
$$

for some positive constant $C_{T}$.

The corner problem (1) is said to be ill-posed (or to generate a violent instability) if it is not strongly well-posed and if such integers do not exist.

The main theorem of this paper is the following :

THEOREM 14. Let (1) be a corner problem satisfying Hypothesis 1-2-21 and 22 then (1) can not be weakly well-posed. In other words, for all $K, K_{2} \in \mathbb{N}, f \in H^{K}\left(\Omega_{T}\right), g_{2} \in H^{K_{2}}\left(\Omega_{T}\right)$ and for all $K_{1} \in \mathbb{N}^{*}$ one can find $g_{1} \in H^{K_{1}}\left(\partial \Omega_{1, T}\right)$, such that the energy estimate

$$
\|u\|_{L^{2}\left(\Omega_{T}\right)}^{2}+\left\|u_{\mid x_{1}=0}\right\|_{L^{2}\left(\partial \Omega_{1, T}\right)}^{2}+\left\|u_{\mid x_{2}=0}\right\|_{L^{2}\left(\partial \Omega_{2, T}\right)}^{2} \leq C_{T}\left(\|f\|_{H^{K}\left(\Omega_{T}\right)}^{2}+\left\|g_{1}\right\|_{H^{K_{1}\left(\partial \Omega_{1, T}\right)}}^{2}+\left\|g_{2}\right\|_{H^{K_{2}\left(\partial \Omega_{2, T}\right)}}^{2}\right),
$$

is violated.

The proof of Theorem 14 is given in Section 8. This proof is based on the rigorous construction of geometric optics expansions for the corner problem (1) with an arbitrary number of amplifications compared to the source term on the side $\partial \Omega_{1}$. This construction, which is the technical part of the proof, is made in Section 7.

6. General framework. In this section we recall some notations and definitions used in [4] to describe rigorously the set of phases obtained by reflection against the side of the quarter space.

6.1. Definition of the frequency set and first properties. Let us start with the definition of what we mean by a frequency set:

Definition 15. Let $\mathscr{I}$ be a subset of $\mathbb{N}$ and $\underline{\tau} \in \mathbb{R}, \underline{\tau} \neq 0$. A set indexed by $\mathscr{I}$,

$$
\mathscr{F}:=\left\{f^{i}:=\left(\underline{\tau}, \xi_{1}^{i}, \xi_{2}^{i}\right), i \in \mathscr{I}\right\},
$$

will be a frequency set for the corner problem (1) if for all $i \in \mathscr{I}, f_{i}$ satisfies

$$
\operatorname{det} \mathscr{L}\left(f^{i}\right)=0 \text {, }
$$

and one of the following alternatives:

i) $\xi_{1}^{i}, \xi_{2}^{i} \in \mathbb{R}$.

ii) $\xi_{1}^{i} \in(\mathbb{C} \backslash \mathbb{R}), \xi_{2}^{i} \in \mathbb{R}$ and $\operatorname{Im} \xi_{1}^{i}>0$.

iii) $\xi_{2}^{i} \in(\mathbb{C} \backslash \mathbb{R}), \xi_{1}^{i} \in \mathbb{R}$ and $\operatorname{Im} \xi_{2}^{i}>0$.

In all what follows, if $\mathscr{F}$ is a frequency set for the corner problem (1), we will define:

$$
\begin{aligned}
\mathscr{F}_{o s} & \left.:=\left\{f^{i} \in \mathscr{F} \text { satisfying } i\right)\right\}, \\
\mathscr{F}_{\text {ev } 1}: & \left.=\left\{f^{i} \in \mathscr{F} \text { satisfying } i i\right)\right\}, \\
\mathscr{F}_{\text {ev } 2}: & \left.=\left\{f^{i} \in \mathscr{F} \text { satisfying } i i i\right)\right\} .
\end{aligned}
$$

It is clear that the sets $\mathscr{F}_{o s}, \mathscr{F}_{\text {ev } 1}$ and $\mathscr{F}_{\text {ev } 2}$ give a partition of $\mathscr{F}$. Moreover to each $f^{i} \in \mathscr{F}_{o s}$, we can associate a group velocity $v_{i}:=\left(v_{i, 1}, v_{i, 2}\right)$. Let us recall that the group velocity $v_{i}$ has beeen introduced in 
Definition 5. The set $\mathscr{F}_{\text {os }}$ can thus be decomposed as follows:

$$
\begin{aligned}
\mathscr{F}_{i i} & :=\left\{f^{i} \in \mathscr{F}_{o s} \backslash v_{i, 1}, v_{i, 2}>0\right\}, \mathscr{F}_{i o}:=\left\{f^{i} \in \mathscr{F}_{o s} \backslash v_{i, 1}>0, v_{i, 2}<0\right\}, \\
\mathscr{F}_{o i} & :=\left\{f^{i} \in \mathscr{F}_{o s} \backslash v_{i, 1}<0, v_{i, 2}>0\right\}, \mathscr{F}_{o o}:=\left\{f^{i} \in \mathscr{F}_{o s} \backslash v_{i, 1}, 0, v_{i, 2}<0\right\} . \\
\mathscr{F}_{g} & :=\left\{f^{i} \in \mathscr{F}_{o s} \backslash v_{i, 1}=0 \text { or } v_{i, 2}=0\right\} .
\end{aligned}
$$

The partition of $\mathscr{F}$ induces the following partition of $\mathscr{I}$ :

$$
\mathscr{I}=\mathscr{I}_{g} \cup \mathscr{I}_{o o} \cup \mathscr{I}_{i o} \cup \mathscr{I}_{o i} \cup \mathscr{I}_{i i} \cup \mathscr{I}_{e v 1} \cup \mathscr{I}_{e v 2}
$$

where we have denoted by $\mathscr{I}_{i o}$ (resp. $g,{ }_{o o},{ }_{o i},{ }_{i i}$, ev1, ev2) the set of indices $i \in \mathscr{I}$ such that the corresponding frequency $f^{i} \in \mathscr{F}_{i o}$ (resp. $g, o o, o i, i i, e v 1, e v 2$ ).

From now on, the source term $g_{1}^{\varepsilon}$ on the $\partial \Omega_{1}$ boundary in (1) reads :

$$
g_{1}^{\varepsilon}\left(t, x_{2}\right):=e^{\frac{i}{\varepsilon}\left(\underline{\tau} t+\underline{\xi}_{2} x_{2}\right)} \varepsilon^{M+1} g\left(t, x_{2}\right)
$$

for some fixed integer $M$ and where the amplitude $g$ has its support in space away from the corner and is zero for negative times.

The following definition gives a precise framework for the phase generation process described in paragraph 4. More precisely, this definition qualifies the frequency set that contains all (and only) the frequencies linked with the expected non-zero amplitudes in the WKB expansion:

DEFINITION 16. The corner problem (1) is said to be complete for reflections if there exists a set of frequencies $\mathscr{F}$ satisfying the following properties:

i) $\mathscr{F}$ contains the real roots (in the variable $\xi_{1}$ ) associated with incoming-outgoing or incoming-incoming group velocities and the complex roots with positive imaginary part, to the dispersion relation

$$
\operatorname{det} \mathscr{L}\left(\underline{\tau}, \xi_{1}, \underline{\xi}_{2}\right)=0 \text {. }
$$

ii) $\mathscr{F}_{g}=\emptyset$.

iii) If $\left(\underline{\tau}, \xi_{1}^{i}, \xi_{2}^{i}\right) \in \mathscr{F}_{i o}$, then $\mathscr{F}$ contains all the roots (in the $\xi_{2}$-variable), denoted by $\xi_{2}^{p}$, to the dispersion relation $\operatorname{det} \mathscr{L}\left(\underline{\tau}, \xi_{1}^{i}, \xi_{2}\right)=0$, that satisfy one of the following two alternatives:

$\left.i i i^{\prime}\right) \xi_{2}^{p} \in \mathbb{R}$ and the frequency $\left(\underline{\tau}, \xi_{1}^{i}, \xi_{2}^{p}\right)$ is associated with an outgoing-incoming group velocity or an incoming-incoming group velocity.

$\left.i i i^{\prime \prime}\right) \operatorname{Im} \xi_{2}^{p}>0$.

iv) If $\left(\underline{\tau}, \xi_{1}^{i}, \xi_{2}^{i}\right) \in \mathscr{F}_{o i}$, then $\mathscr{F}$ contains all the roots (in the $\xi_{1}$-variable), denoted by $\xi_{1}^{p}$, to the dispersion relation $\operatorname{det} \mathscr{L}\left(\underline{\tau}, \xi_{1}, \xi_{2}^{i}\right)=0$, that satisfy one of the following two alternatives:

$\left.i v^{\prime}\right) \xi_{1}^{p} \in \mathbb{R}$ and the frequency $\left(\underline{\tau}, \xi_{1}^{p}, \xi_{2}^{i}\right)$ is associated with an incoming-outgoing or an incoming-incoming group velocity.

$\left.i v^{\prime \prime}\right) \operatorname{Im} \xi_{1}^{p}>0$.

v) $\mathscr{F}$ is minimal (for the inclusion) for the four preceding properties.

Point $i$ ) imposes that the frequency set $\mathscr{F}$ contains all (and only all) the incoming phases for $\partial \Omega_{1}$ that are induced by the source term $g_{1}^{\varepsilon}$.

Point $i i i)$ (resp. $i v)$ ) explains the generation by reflection on the side $\partial \Omega_{2}$ (resp. $\partial \Omega_{1}$ ) of a wave packet that emanates from the side $\partial \Omega_{1}$ (resp. $\left.\partial \Omega_{2}\right)$.

An immediate consequence of the minimality of $\mathscr{F}$ is that $\mathscr{F}_{o o}$ is empty. Moreover let us stress that according to the discussion made in paragraph 4 , because we are dealing with finite time and a source term which has its support away from the corner, without loss of generality we can assume that $\# \mathscr{F}<+\infty$.

Let us recall that if the corner problem is complete for reflections, one can define two applications, defined on the index set $\mathscr{I}$ and which give, in the output, the indices "in the direct vicinity" of the input index:

$$
\Phi, \Psi: \mathscr{I} \longrightarrow \mathscr{P}_{N}(\mathscr{I})
$$


where $\mathscr{P}_{N}(\mathscr{I})$ denotes the power set of $\mathscr{I}$ with at most $N$ elements. More precisely, the definitions of $\Phi$ and $\Psi$ are: for $i \in \mathscr{I}, f^{i}=\left(\underline{\tau}, \xi_{1}^{i}, \xi_{2}^{i}\right)$,

$$
\Phi(i):=\left\{j \in \mathscr{I} \backslash \xi_{2}^{j}=\xi_{2}^{i}\right\} \text { and } \Psi(i):=\left\{j \in \mathscr{I} \backslash \xi_{1}^{j}=\xi_{1}^{i}\right\} .
$$

For convenience let us introduce the short-hand notations:

$$
\Phi^{*}(i):=\Phi(i) \backslash\{i\} \text { and } \Psi^{*}(i):=\Psi(i) \backslash\{i\}
$$

Thanks to these functions, the index set $\mathscr{I}$ can be seen as a graph. This graph structure will be more abstract than the desciption of $\mathscr{I}$ based on the wave packet reflections, but it will be easier to handle with when we will construct the WKB expansion. This graph structure is defined by the following relation : two points $i, j \in \mathscr{I}$ are linked by an edge if and only if $i \in \Phi(j)$ or $i \in \Psi(j)$.

In terms of wave packet reflection, the set $\Phi(i)$ (resp. $\Psi(i)$ ) is the set of all indices of the phases that are considered in the reflection of the wave packet with phase $f^{i}$ on $\partial \Omega_{2}$. Let us stress that the index $i$ is not necessarily the index of an incident ray but can be the index of one of the reflected rays. Applications $\Phi$ and $\Psi$ satisfy the following properties:

PROPOSITION 17. If the corner problem (1) is complete for reflections, then $\Phi$ and $\Psi$ satisfy:

i) $\forall i \in \mathscr{I}, i \in \Psi(i), i \in \Phi(i)$.

ii) $\forall i \in \mathscr{I}, \forall j \in \Psi(i), \forall k \in \Phi(i)$ we have $\Psi(i)=\Psi(j)$ and $\Phi(i)=\Phi(k)$.

iii) $\forall i \in \mathscr{I}, \Phi(i) \cap \mathscr{I}_{\text {ev } 2}=\emptyset$ and $\Psi(i) \cap \mathscr{I}_{\text {ev } 1}=\emptyset$. And, $\forall i \in \mathscr{I}_{\text {ev } 1}, \forall j \in \mathscr{I}_{\text {ev } 2}$, we have $\Psi(i) \subset \mathscr{I}_{\text {ev } 1}$, $\Phi(i) \subset \mathscr{I}_{\text {ev } 2}$.

iv $) \forall i \in \mathscr{I}_{o s}, \#\left(\Phi(i) \cap \mathscr{I}_{\text {ev } 1} \cap \mathscr{I}_{i o} \cap \mathscr{I}_{i i}\right) \leq p_{1}$, and $\#\left(\Psi(i) \cap \mathscr{I}_{\text {ev } 2} \cap \mathscr{I}_{\text {oi }} \cap \mathscr{I}_{i i}\right) \leq p_{2}$.

$v) \forall i \in \mathscr{I}$, we have on the one hand $\forall i_{1}, i_{2} \in \Phi(i), i_{1} \neq i_{2}$ :

$$
\Phi(i) \cap \Psi\left(i_{1}\right)=\left\{i_{1}\right\} \text { and } \Psi\left(i_{1}\right) \cap \Psi\left(i_{2}\right)=\emptyset,
$$

and on the other hand, $\forall j_{1}, j_{2} \in \Psi(i), j_{1} \neq j_{2}$ :

$$
\Psi(i) \cap \Phi\left(j_{1}\right)=\left\{j_{1}\right\} \text { and } \Phi\left(j_{1}\right) \cap \Phi\left(j_{2}\right)=\emptyset .
$$

We refer to [4] for a proof of this proposition.

Thanks to applications $\Phi$ and $\Psi$ it is easy to define the notion of two linked indices in the graph structure of $\mathscr{I}$ :

Definition 18. If $\underline{i} \in \mathscr{I}_{\text {io }}$, we say that the index $j \in \mathscr{I}_{i o} \cup \mathscr{I}_{\text {ev1 } 1}$ (resp. $j \in \mathscr{I}_{\text {oi }} \cup \mathscr{I}_{\text {ev } 2}$ ) is linked with the index $\underline{i}$, if there exist $p \in 2 \mathbb{N}+1$ (resp. $p \in 2 \mathbb{N}$ ) and a sequence of indices $\ell=\left(\ell_{1}, \ell_{2}, \ldots, \ell_{p}\right) \in \mathscr{I}^{p}$ such that: $\left.\alpha^{\prime}\right) \ell_{1} \in \Psi(\underline{i}) \cap \mathscr{I}_{o i}, \ell_{2} \in \Phi\left(\ell_{1}\right) \in \mathscr{I}_{i o}, \ldots, j \in \Phi\left(\ell_{p}\right)$ (resp. $\left.j \in \Psi\left(\ell_{p}\right)\right)$.

We say that the index $j \in \mathscr{I}_{i i}$ is linked with the index $\underline{i}$, if there is a sequence of indices $\ell=\left(\ell_{1}, \ell_{2}, \ldots, \ell_{p}\right) \in$ $\mathscr{I}^{p}$ such that:

$\left.\beta^{\prime}\right) \ell_{1} \in \Psi(\underline{i}) \cap \mathscr{I}_{\text {oi }}, \ell_{2} \in \Phi\left(\ell_{1}\right) \cap \mathscr{I}_{i o}, \ldots,\left\{\begin{array}{l}j \in \Phi\left(\ell_{p}\right), \quad p \text { is odd, } \\ j \in \Psi\left(\ell_{p}\right), \quad p \text { is even. }\end{array}\right.$

If $\underline{i} \in \mathscr{I}_{\text {oi }}$, we say that the index $j \in \mathscr{I}_{i o} \cup \mathscr{I}_{\text {ev1 }}$ (resp. $j \in \mathscr{I}_{\text {oi }} \cup \mathscr{I}_{\text {ev2 }}$ ) is linked with the index $\underline{i}$, if there exist $p \in 2 \mathbb{N}$ (resp. $p \in 2 \mathbb{N}+1$ ) and a sequence of indices $\ell=\left(\ell_{1}, \ell_{2}, \ldots, \ell_{p}\right) \in \mathscr{I}^{p}$ such that: $\left.\alpha^{\prime \prime}\right) \ell_{1} \in \Phi(\underline{i}) \cap \mathscr{I}_{i o}, \ell_{2} \in \Psi\left(\ell_{1}\right) \in \mathscr{I}_{o i}, \ldots, j \in \Phi\left(\ell_{p}\right)$ (resp. $\left.j \in \Psi\left(\ell_{p}\right)\right)$.

We say that the index $j \in \mathscr{I}_{i i}$ is linked with the index $\underline{i}$, if there exists a sequence of indices $\ell=$ $\left(\ell_{1}, \ell_{2}, \ldots, \ell_{p}\right) \in \mathscr{I}^{p}$ such that:

$\left.\beta^{\prime \prime}\right) \ell_{1} \in \Phi(\underline{i}) \cap \mathscr{I}_{i o}, \ell_{2} \in \Psi\left(\ell_{1}\right) \cap \mathscr{I}_{o i}, \ldots, \begin{cases}j \in \Psi\left(\ell_{p}\right), & p \text { is odd, } \\ j \in \Phi\left(\ell_{p}\right), & p \text { is even. }\end{cases}$ 
Finally, if $\underline{i} \in \mathscr{I}_{i i} \cup \mathscr{I}_{\text {ev1 }} \cup \mathscr{I}_{\text {ev } 2}$, we say that there is no element of $\mathscr{I}$ linked with $\underline{i}$.

Moreover, we will say that an index $j \in \mathscr{I}$ is linked with the index $\underline{i}$ by a sequence of type $H$ (for "horizontal") (resp. V (for "vertical")) and we will note $i \underset{H}{\longrightarrow} j$ (resp. $i \underset{V}{\longrightarrow} j)$ if the sequence $\left(\underline{i}, \ell_{1}, \ell_{2}, \ldots, \ell_{p}, j\right)$ satisfies $\left.\alpha^{\prime \prime}\right)$ or $\left.\beta^{\prime \prime}\right)\left(\right.$ resp. $\left.\alpha^{\prime}\right)$ or $\left.\beta^{\prime}\right)$ ).

Applications $\Phi$ and $\Psi$ also enable us to define a set of class of representative for the complex valued frequencies (or evanescent frequencies) in view to treat these frequencies in a "monoblock" way as it is done in [12]. That is to say that for an index $\underline{i} \in \mathscr{I}_{e v 1}$ (resp. $\underline{i} \in \mathscr{I}_{\text {ev2 }}$ ), all the elements $j \in \mathscr{I}_{\text {ev1 }} \cap \Phi(\underline{i})$ (resp. $j \in \mathscr{I}_{e v 2} \cap \Psi(\underline{i})$ ) will contribute to a single vector valued amplitude. To write off the ansatz and to describe with enough precision the boundary conditions, it is useful to introduce the two equivalence relations $\underset{\Phi}{\sim}$ and $\widetilde{\Psi}$ defined by:

$$
i \underset{\Phi}{\sim} j \Longleftrightarrow j \in \Phi(i) \text {, and } i \underset{\Psi}{\sim} j \Longleftrightarrow j \in \Psi(i) .
$$

Let $\mathfrak{C}_{1}$ (resp. $\left.\mathfrak{C}_{2}\right)$ be the set of equivalence classes for the relation $\underset{\Phi}{\sim}($ resp. $\underset{\Psi}{\sim})$, and $\mathscr{R}_{1}$ (resp. $\mathscr{R}_{2}$ ), be a set of class representative for $\mathfrak{C}_{1}$ (resp. $\mathfrak{C}_{2}$ ). So $\mathscr{R}_{1}$ (resp. $\mathscr{R}_{2}$ ) is a set of indices which includes all the possible values for $\xi_{2}$ (resp. $\xi_{1}$ ) of the different frequencies. We also define $\mathfrak{R}_{1}$ and $\mathfrak{R}_{2}$ by:

$$
\begin{aligned}
& \mathfrak{R}_{1}:=\left\{i \in \mathscr{R}_{1} \backslash \Phi(i) \cap \mathscr{I}_{\text {ev } 1} \neq \emptyset\right\}, \\
& \mathfrak{R}_{2}:=\left\{i \in \mathscr{R}_{2} \backslash \Psi(i) \cap \mathscr{I}_{\text {ev } 2} \neq \emptyset\right\} .
\end{aligned}
$$

$\mathfrak{R}_{1}$ (resp. $\mathfrak{R}_{2}$ ) is a set of class representative of the values in $\xi_{2}$ (resp. $\xi_{1}$ ) for which there is an evanescent mode for the side $\partial \Omega_{1}$ (resp. $\partial \Omega_{2}$ ). At last, without loss of generality, we can always assume that $n_{1} \in \mathscr{R}_{2}$, in other words, we choose $n_{1}$ as a class representative of its own equivalence class.

To conclude, let us recall the following proposition which is an immediate consequence of Definitions 4.2 and 4.3 .

Proposition 19. Let $\mathscr{F}$ be a complete for reflections frequency set indexed by $\mathscr{I}$. Let $\mathscr{I}_{0}$ be the set of indices in $\mathscr{I}$ generated by the source term $g_{1}^{\varepsilon}$, that is to say:

$$
\mathscr{I}_{0}:=\left\{i \in \mathscr{I}_{i o} \cup \mathscr{I}_{i i} \cup \mathscr{I}_{\text {ev } 1} \backslash \operatorname{det} \mathscr{L}\left(\underline{\tau}, \xi_{1}^{i}, \underline{\xi_{2}}\right)=0\right\} .
$$

Let $\mathscr{I}_{\mathscr{R}}$ be the set of indices in $\mathscr{I}$ linked with one of the elements of $\mathscr{I}_{0}$. Then

$$
\mathscr{I}_{\mathscr{R}}=\mathscr{I} \text {. }
$$

6.2. Frequency sets with loop. As in [4] we will assume that the considered corner problem admits a unique selfinteraction loop ${ }^{4}$. Let us now give the definition of the loop that has been mentionned in Section 4:

Definition 20. Let $i \in \mathscr{I}, p \in 2 \mathbb{N}+1$ and $\ell=\left(\ell_{1}, \ldots, \ell_{p}\right) \in \mathscr{I}^{p}$.

- We say that the index $i \in \mathscr{I}$ admits a loop if there exists a sequence $\ell$ satisfying:

$$
\ell_{1} \in \Phi(i), \ell_{2} \in \Psi\left(\ell_{1}\right), \ldots, i \in \Psi\left(\ell_{p}\right) .
$$

- A loop for an index $i$ is said to be simple if the sequence $\ell$ does not contain a periodically repeated subsequence.

- An index $i \in \mathscr{I}_{\text {io }}$ (resp. $i \in \mathscr{I}_{\text {oi }}$ ) admits a selfinteraction loop if $i$ admits a simple loop and if the sequence $(i, \ell, i)$ is of type $V$ (resp. H) according to Definition 18.

Let us assume that:

Hypothesis 21. Let (1) be complete for reflections, we assume that the frequency set $\mathscr{F}$ contains a unique loop, of size 3 and that this loop is a selfinteraction loop. More precisely, we ask that the following properties are satisfied:

vi) $\exists\left(n_{1}, n_{3}\right) \in \mathscr{I}_{i o}^{2},\left(n_{2}, n_{4}\right) \in \mathscr{I}_{o i}^{2}$ such that

$$
n_{2} \in \Psi\left(n_{1}\right), n_{3} \in \Phi\left(n_{2}\right), n_{4} \in \Psi\left(n_{3}\right), n_{1} \in \Phi\left(n_{4}\right) .
$$

vii) Let $i \in \mathscr{I}$ an index with a loop $\ell=\left(\ell_{1}, \ldots, \ell_{p}\right)$. Then $p=3$ and $\left\{i, \ell_{1}, \ell_{2}, \ell_{3}\right\}=\left\{n_{1}, n_{2}, n_{3}, n_{4}\right\}$.

\footnotetext{
${ }^{4}$ Compared to [4], for convenience, we have exchanged the indices $n_{2}$ and $n_{4}$. It is due to the fact that here we are more interested in a precise description in terms of wave packets reflection than in [4].
} 


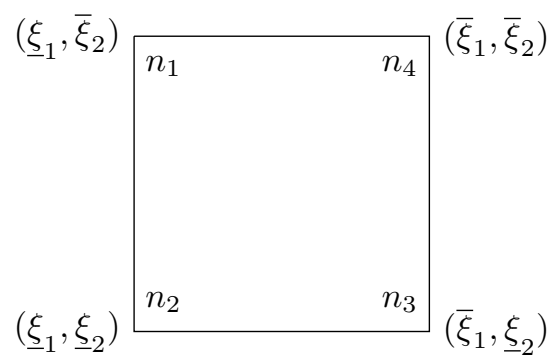

FIG. 2. The coefficients of the loop's elements.

$$
f^{n_{1}}:=\left(\underline{\tau}, \underline{\xi}_{1}, \bar{\xi}_{2}\right), f^{n_{2}}:=\left(\underline{\tau}, \underline{\xi}_{1}, \underline{\xi}_{2}\right), f^{n_{3}}:=\left(\underline{\tau}, \bar{\xi}_{1}, \underline{\xi}_{2}\right) \text { and, } f^{n_{4}}:=\left(\underline{\tau}, \bar{\xi}_{1}, \bar{\xi}_{2}\right) .
$$

We also assume that the only index of $\mathscr{I}$ for which uniform Kreiss-Lopatinskii condition is violated is $n_{1}$. We summarize the previous requests on the set of indices for the corner problem (1) in the following Hypothesis (which specifies Hypothesis 2.3):

Hypothesis 22. The corner problem (1) is in the class $W R$ for the side $\partial \Omega_{1}$ and satisfies the uniform Kreiss-Lopatinskii condition on the side $\partial \Omega_{2}$. The set where the uniform Kreiss-Lopatinskii condition for the side $\partial \Omega_{1}$ breaks down $\Upsilon$ (see Definition 8) is given by $\Upsilon=\left\{\widetilde{f}^{n_{1}}:=\left(i \underline{\tau}, \underline{\xi}_{1}, \bar{\xi}_{2}\right)\right\}$.

Moreover to make sure that the transport along the boundary spreads the information away from the corner we will ask that $\underline{\tau}$ and $\bar{\xi}_{2}$ have opposite signs.

To conclude let us remark that when the frequency set $\mathscr{F}$, indexed by $\mathscr{I}$, is complete for the reflections and that when it admits a unique loop, then it follows from the definition of linked indices 18 that we have the following propositions: Firstly

Proposition 23. Let $i \in \mathscr{I}$ then there exists a unique type $V$ sequence linking $n_{1}$ to $i$.

$$
\text { Moreover, one can write } \mathscr{I} \backslash\left\{n_{1}, n_{2}, n_{3}, n_{4}\right\} \text { as a partition: }
$$

$$
\left(\mathscr{I} \backslash\left\{n_{1}, n_{2}, n_{3}, n_{4}\right\}\right)=\left(\cup_{l \leq \complement_{1}} A_{a_{l}}\right) \bigcup\left(\cup_{m \leq \complement_{2}} B_{b_{m}}\right) \bigcup\left(\cup_{q \leq \complement_{3}} C_{c_{q}}\right) \bigcup\left(\cup_{r \leq \complement_{4}} D_{d_{r}}\right)
$$

where $A_{a_{l}}$ denotes the set of indices $i \in \mathscr{I}$ from which the type $V$ sequence linking $n_{1}$ to $i$ starts by $a_{l}$, B $b_{b_{m}}$ denotes the set of indices $i \in \mathscr{I}$ from which the type $V$ sequence linking $n_{1}$ to $i$ starts by $\left(n_{2}, b_{l}\right), C_{c_{q}}$ denotes the set of indices $i \in \mathscr{I}$ from which the type $V$ sequence linking $n_{1}$ to $i$ starts by $\left(n_{2}, n_{3}, c_{q}\right)$ and $D_{d_{r}}$ is the set of indices $i \in \mathscr{I}$ such that the type $V$ sequence linking $n_{1}$ to $i$ starts by $\left(n_{2}, n_{3}, n_{4}, d_{r}\right)$.

Secondly

Proposition 24. Let $\mathscr{F}$ be complete for the reflections, under Assumption 21. Let $\mathscr{I}$ be the index set; then $\Phi$ and $\Psi$ satisfy, in addition to the properties of Proposition 1\%, the four extra properties: viii)

$$
\begin{array}{ll}
\Phi\left(n_{1}\right) \backslash\left\{n_{2}\right\} \subset \mathscr{I}_{i o} \cup \mathscr{I}_{i i} \cup \mathscr{I}_{\text {ev } 1}, & \Psi\left(n_{1}\right) \backslash\left\{n_{1}\right\} \subset \mathscr{I}_{o i} \cup \mathscr{I}_{i i} \cup \mathscr{I}_{\text {ev } 2}, \\
\Phi\left(n_{4}\right) \backslash\left\{n_{4}\right\} \subset \mathscr{I}_{i o} \cup \mathscr{I}_{i i} \cup \mathscr{I}_{\text {ev } 1}, & \Psi\left(n_{3}\right) \backslash\left\{n_{3}\right\} \subset \mathscr{I}_{o i} \cup \mathscr{I}_{i i} \cup \mathscr{I}_{\text {ev } 2} .
\end{array}
$$

ix) Let $i \in \mathscr{I}_{i i} \cup \mathscr{I}_{\text {ev } 1}$ and $j \in \mathscr{I}_{i i} \cup \mathscr{I}_{\text {ev } 2}$ then

$$
\begin{aligned}
& i \in \Phi\left(n_{1}\right) \Longrightarrow \Psi(i)=\{i\}, \quad j \in \Psi\left(n_{1}\right) \Longrightarrow \Phi(j)=\{j\}, \\
& i \in \Phi\left(n_{4}\right) \Longrightarrow \Psi(i)=\{i\}, \quad j \in \Psi\left(n_{3}\right) \Longrightarrow \Phi(j)=\{j\} .
\end{aligned}
$$

Let $\underline{j} \in A_{\underline{a}} \backslash\{\underline{a}\}$, we denote by $\ell=\left(\ell_{1}, \ldots \ell_{p}\right)$ the sequence of type $H$ linking $\underline{j}$ to $\underline{a}$. Then, according to the parity of $p$, we have:

$\left.x^{\prime}\right)$ If $p \in 2 \mathbb{N}$, then $\underline{j} \notin \mathscr{I}_{\text {oi }}$. Moreover, if $\underline{j} \in \mathscr{I}_{\text {ev } 1} \cup \mathscr{I}_{i i}$ then $\Psi(\underline{j})=\{\underline{j}\}$.

$\left.x^{\prime \prime}\right)$ If $p \in 2 \mathbb{N}+1$, then $\underline{j} \notin \mathscr{I}_{i o}$. Moreover, if $\underline{j} \in \mathscr{I}_{\text {ev } 2} \cup \mathscr{I}_{i i}$ then $\Phi(\underline{j})=\{\underline{j}\}$. 


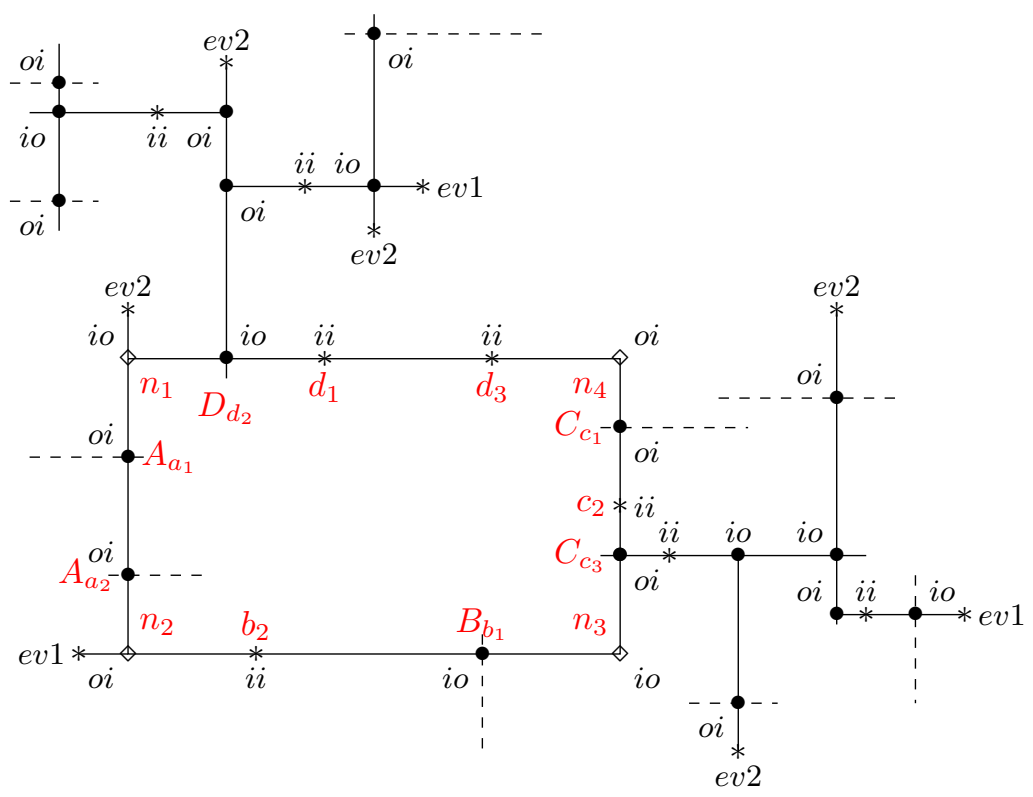

FIG. 3. "Tree structure" of the frequency set $\mathscr{F}$.

Propositions 23 and 24 then permit to show that the set of indices $\mathscr{I}$ has the "tree" structure depicted in the figure 2 .

To conclude this section we define the following matrices which will be useful during the construction of the geometric optics expansions.

Definition 25. For $j=1,2$ and $k \in \mathfrak{R}_{j}$. Let $f^{k}=\left(\underline{\tau}, \xi_{1}^{k}, \xi_{2}^{k}\right)$ be the associated frequency with the index $k$. We define $P_{e v, j}^{k}$ (resp. $\left.Q_{e v, j}^{k}\right)$ the projector on $E_{j}^{s, e}\left(i \underline{\tau}, \xi_{3-j}^{k}\right)\left(\right.$ resp. $\left.A_{j} E_{j}^{s, e}\left(i \underline{\tau}, \xi_{3-j}^{k}\right)\right)$ with respect to the decomposition (27) (resp. (28)).

For $j=1,2$ and $k \in \mathscr{I}_{\text {os }}$. Let $f^{k}=\left(\underline{\tau}, \xi_{1}^{k}, \xi_{2}^{k}\right)$ be the associated frequency with the index $k$. We define $P_{j}^{k}\left(\right.$ resp. $\left.Q_{j}^{k}\right)$ the projector on $\operatorname{ker} \mathscr{L}\left(f^{k}\right)$ (resp. $A_{j} \operatorname{ker} \mathscr{L}\left(f^{k}\right)$ ) with respect to the decomposition (27) (resp. (28)). We also define $R_{j}^{k}$ the partial-inverse of $\mathscr{L}\left(f^{k}\right)$ uniquely determined by:

$$
R_{j}^{k} \mathscr{L}\left(f^{k}\right)=I-P_{j}^{k}, P_{j}^{k} R_{j}^{k}=R_{j}^{k} Q_{j}^{k}=0 .
$$

7. Geometric optics expansions for selfinteracting problems in the $W R$ class. The corner problem that we are now interested in reads:

$$
\begin{cases}L(\partial) u^{\varepsilon}=0, & \text { on } \Omega_{T}, \\ B_{1} u_{\mid x_{1}=0}^{\varepsilon}=g_{M}^{\varepsilon}, & \text { on } \partial \Omega_{1, T}, \\ B_{2} u_{\mid x_{2}=0}^{\varepsilon}=0, & \text { on } \partial \Omega_{2, T}, \\ u_{\mid t \leq 0}^{\varepsilon}=0, & \text { on } \Omega,\end{cases}
$$

where the source term $g_{M}^{\varepsilon}$ is given by:

$$
g_{M}^{\varepsilon}\left(t, x_{2}\right):=\varepsilon^{M+1} e^{\frac{i}{\varepsilon}\left(\underline{\tau} t+\bar{\xi}_{2} x_{2}\right)} g\left(t, x_{2}\right),
$$

with $\underline{\tau}>0$ fixed, $\bar{\xi}_{2}<0$ defined in (44) and where $M \in \mathbb{N} \backslash\{0\}$ is fixed ${ }^{5}$. The function $g$ is zero for negative times and has its support in space away from the corner for all positive times. More precisely, there exists $\underline{y}_{0}>0$ such that:

$$
\forall t \in \mathbb{R}_{+}, \forall x_{2}<\underline{y}_{0}, g\left(t, x_{2}\right)=0 .
$$

\footnotetext{
${ }^{5}$ The case $M=0$ corresponds to the boundary value problem in the half space and will not be discussed here. We refer to [8] for a study in such a configuration.
} 
The aim of this section is to construct the geometric optics expansion associated to the corner problem (47) up to a fixed time $T>0$. More precisely, we want to show that, up to choose the support of the function $g$ close enough to the corner, the geometric optics expansion suffers a number of amplifications that can be arbitrarily large. Indeed, due to the presence of the loop, the amplification arising for the amplitude associated to $n_{1}$ will be repeated at each cycle around the loop. More precisely, if one sets $M$ in (48) and wants to show that the leading order in the WKB expansion is of order zero then it will be sufficient to choose $g$ such that its support is close enough to the corner to ensure that $M$ complete circuits has been made before the time $T$.

Let us give more details about these times of travel. We fix a point $\left(0, y^{0}\right) \in \partial \Omega_{1}$ such that $g\left(0, y^{0}\right) \neq 0$ and we draw the characteristic with group velocity $v_{n_{1}}$ passing through $\left(0, \underline{y}^{0}\right)$. This characteristic intersects $\partial \Omega_{2}$ in a point $\left(\underline{x}^{0}, 0\right)$ after a certain time of travel $t_{1}\left(y^{0}\right)$. In a second time we draw the characteristic with group velocity $v_{n_{2}}$ passing through $\left(\underline{x}^{0}, 0\right)$. It intersects $\partial \Omega_{1}$ in a point $\left(0, y^{1}\right)$ after a time $t_{2}\left(y^{0}\right)$. We repeat the same process for the characteristics with group velocities $v_{n_{3}}$ and $v_{n_{4}}$. Let $\left(\underline{x}^{1}, 0\right),\left(0, \underline{y}^{2}\right)$ be the corresponding points of intersection and $t_{3}\left(\underline{y}^{0}\right), t_{4}\left(\underline{y}^{0}\right)$ be the corresponding times of travel. An easy computation shows that:

$$
\underline{y}^{2}:=\beta \underline{y}^{0}, \text { and, } T_{1}\left(\underline{y}^{0}\right):=\sum_{j=1}^{4} t_{j}\left(\underline{y}^{0}\right)=\alpha \underline{y}^{0},
$$

where the scalars $\alpha$ and $\beta$ are given by:

$$
\alpha:=-\frac{1}{v_{n_{1}, 2}}\left(1-\frac{v_{n_{1}, 1}}{v_{n_{2}, 1}}+\frac{v_{n_{1}, 1} v_{n_{2}, 2}}{v_{n_{2}, 1} v_{n_{3}, 2}}-\frac{v_{n_{1}, 1} v_{n_{2}, 2} v_{n_{3}, 1}}{v_{n_{2}, 1} v_{n_{3}, 2} v_{n_{4}, 1}}\right),
$$

$$
\beta:=\prod_{j=1}^{4} \beta_{j}, \text { with, } \beta_{j}:=\left\{\begin{array}{l}
\frac{v_{n_{j}, 1}}{v_{n_{j},},}, \text { if } j \text { is odd } \\
\frac{v_{n_{j}, 2}}{v_{n_{j}, 1}}, \text { if } j \text { is even }
\end{array}\right.
$$

and where the $v_{n_{j}}:=\left[\begin{array}{ll}v_{n_{j}, 1} & v_{n_{j}, 2}\end{array}\right]$ are the group velocities for the indices of the loop.

After $m \in \mathbb{N}^{*}$ circuits around the loop the initial point $\left(0, \underline{y}^{0}\right)$ comes back on $\partial \Omega_{1}$ in $\left(0, \underline{y}^{2 m}\right)$ after a total time of travel $\underline{T}_{m}$. It is easy to show that :

$$
\underline{y}^{2 m}:=\beta^{m} \underline{y}^{0} \text {, and, } \underline{T}_{m}=\underline{T}_{m}\left(\underline{y}^{0}\right):=\alpha \underline{y}^{0} \sum_{k=0}^{m-1} \beta^{k} .
$$

We point the fact that if $\beta<1$, that is to say when the rays get closer and closer to the corner, the limit of the sum defining $\underline{T}_{m}$ when $m$ goes to infinity is finite. In other words, the rays reach the corner in a finite time $T_{\max }:=\alpha \underline{y}^{0} \sum_{k=0}^{\infty} \beta^{k}$. In this particular situation we will thus assume that $T<T_{\max }$.

It is clear that one can always choose $\underline{y}^{0}$ in such a way that:

$$
\underline{T}_{M} \leq T<\underline{T}_{M+1} .
$$

As already mentioned, if $t \in\left[\underline{T}_{k}, \min \left(\underline{T}_{k+1}, T\right)\right.$ [ one expects all the amplitudes of order less than $M-k+1$ to be zero. Moreover it is also expected that the traces on $\partial \Omega_{1}$ of the amplitudes associated to the index $n_{1}$ vanish for $x_{2}<y^{2 k}$. Indeed, for $t \in\left[\underline{T}_{k}, \min \left(\underline{T}_{k+1}, T\right)\right.$ [, the information has only make $k$ complete circuits around the loop, so it can not have been amplified more than $k$ times and can not have been transported under the threshold $y^{2 k}$. This observation motivates the following definition:

Definition 26. Let us write, for $n \in \mathbb{N}$,

$$
u_{n, n_{1}}(t, x):=\nu_{n, n_{1}}(t, x) e+\check{v}_{n}(t, x),
$$

where the vector e has been introduced in Definition 12 (this decomposition will be explained in paragraph 7.1) and where $\check{v}_{n} \in \oplus_{j \in \Phi^{*}\left(n_{1}\right)} \operatorname{ker} \mathscr{L}\left(f^{j}\right)$. For $M \in \mathbb{N}$ we distinguish the two following subcases:

$\diamond$ if $\beta \leq 1$, we say that the sequence of amplitudes $\left(u_{n, n_{1}}\right)_{n \in \mathbb{N}}$ is in $\mathscr{P}_{\leq}$if for all $n \in \mathbb{N}, \nu_{n, n_{1 \mid x_{1}=0}}$ is in $\mathscr{P}_{b, \leq}^{M-n}$. Where the space $\mathscr{P}_{b, \leq}^{M-n}$ is the set of functions $\mu \in \mathscr{C}^{\infty}(]-\infty, T\left[, \mathscr{D}\left(\mathbb{R}_{+}\right)\right)$satisfying:

i) If $M-n>0$, then for $-\infty<t<\underline{T}_{M-n}, \mu\left(t, x_{2}\right)=0 \forall x_{2} \in \mathbb{R}_{+}$, and for all $k$ such that $M-n \leq k \leq M$, if $\underline{T}_{k} \leq t<\min \left(T, \underline{T}_{k+1}\right), \mu\left(t, x_{2}\right)=0 \forall x_{2}<\underline{y}^{2 k}$. 
ii) If $M-n \leq 0$, then $\mu$ is zero for negative times, and for all $k$ such that $0 \leq k \leq M$, if $\underline{T}_{k} \leq t<$ $\min \left(T, \underline{T}_{k+1}\right), \mu(t, x)=0 \forall x_{2}<y^{2 k}$.

iii) If $n \leq-1$ then $\mathscr{P}_{b}^{M-n}=\{0\}$.

$\diamond$ If $\beta>1$, we say that the sequence of amplitudes $\left(u_{n, n_{1}}\right)_{n \in \mathbb{N}}$ is in $\mathscr{P}_{>}$if for all $n \in \mathbb{N}, \nu_{n, n_{1 \mid x_{1}=0}}$ is in $\mathscr{P}_{b,>}^{M-n}$. Where the space $\mathscr{P}_{b,>}^{M-n}$ is the set of functions $\mu \in \mathscr{C}^{\infty}(]-\infty, T\left[, \mathscr{D}\left(\mathbb{R}_{+}\right)\right)$satisfying:

i) If $M-n>0$, then for $-\infty<t<\underline{T}_{M-n}, \mu\left(t, x_{2}\right)=0 \forall x \in \mathbb{R}_{+}$, and for all $k$ such that $M-n \leq k \leq M$, if $\underline{T}_{k} \leq t<\min \left(T, \underline{T}_{k+1}\right), \mu\left(t, x_{2}\right)=0 \forall x<\underline{y}_{0}$.

ii) If $n \leq-1$ then $\mathscr{P}_{b}^{M-n}=\{0\}$.

The fact that we distinguish the profile space for $\beta \leq 1$ and for $\beta>1$ is due to technical reasons which will be explained in paragraph 7.1.4. Notice that by definition we have $\mathscr{P}_{b, \leq}^{M-n}=\mathscr{P}_{b, \leq}^{0}$ for all $n>M$.

We are now able to describe the expected space of profiles:

DeFinition 27. When $\beta \leq 1$, a sequence $\left(u_{n, k}\right)_{n \in \mathbb{N}, k \in \mathscr{I}_{\text {os }}}$ is said to be in the space of profiles $\mathbb{P}_{\text {os }, \leq}$ if $\left(u_{n, n_{1}}\right) \in \mathscr{P}_{\leq}$and if for all $k \in \mathscr{I}_{\text {os }} \backslash\left\{n_{1}\right\}$, for all $n \in \mathbb{N}$, $u_{n, k}$ lies in $H^{\infty}\left(\Omega_{T}\right)$.

When $\beta>1$, a sequence $\left(u_{n, k}\right)_{n \in \mathbb{N}, k \in \mathscr{I}_{\text {os }}}$ is said to be in the space of profiles $\mathbb{P}_{\text {os },<}$ if $\left(u_{n, n_{1}}\right) \in \mathscr{P}_{<}$and if for all $k \in \mathscr{I}_{\text {os }} \backslash\left\{n_{1}\right\}$, for all $n \in \mathbb{N}$, $u_{n, k}$ lies in $H^{\infty}\left(\Omega_{T}\right)$.

For $i=1,2$, the set $\mathbb{P}_{\text {ev, } i}$ of evanescent profiles for the side $\partial \Omega_{i}$ is the set of $U\left(t, x, X_{i}\right) \in H^{\infty}\left(\Omega_{T} \times \mathbb{R}_{+}\right)$ for which there exists a positive $\delta$ such that $e^{\delta X_{i}} U\left(t, x, X_{i}\right) \in H^{\infty}\left(\Omega_{T} \times \mathbb{R}_{+}\right)$.

As already mentioned, we will have to consider three kinds of phases, the oscillating ones, the evanescent ones for the side $\partial \Omega_{1}$ and the evanescent ones for the side $\partial \Omega_{2}$. According to their kind the phases be denoted by:

$$
\begin{aligned}
\varphi_{k}(t, x) & :=\left\langle(t, x), f^{k}\right\rangle, f^{k} \in \mathscr{F}_{o s}, \\
\psi_{k, 1}\left(t, x_{2}\right) & :=\left\langle\left(t, 0, x_{2}\right), f^{k}\right\rangle, f^{k} \in \mathscr{F}_{\text {ev } 1} \cup \mathscr{F}_{o s}, \\
\psi_{k, 2}\left(t, x_{1}\right) & :=\left\langle\left(t, x_{1}, 0\right), f^{k}\right\rangle, f^{k} \in \mathscr{F}_{\text {ev } 2} \cup \mathscr{F}_{o s} .
\end{aligned}
$$

Once the expected phases are defined, we postulate the ansatz:

$$
\begin{aligned}
u^{\varepsilon}(t, x) & \sim \sum_{n \geq 0} \sum_{k \in \mathscr{I}_{o s}} \varepsilon^{n} e^{\frac{i}{\varepsilon} \varphi_{k}(t, x)} u_{n, k}(t, x) \\
& +\sum_{n \geq 0} \sum_{k \in \Re_{1}} \varepsilon^{n} e^{\frac{i}{\varepsilon} \psi_{k, 1}\left(t, x_{2}\right)} U_{n, k, 1}\left(t, x, \frac{x_{1}}{\varepsilon}\right)+\sum_{n \geq 0} \sum_{k \in \Re_{2}} \varepsilon^{n} e^{\frac{i}{\varepsilon} \psi_{k, 2}\left(t, x_{1}\right)} U_{n, k, 2}\left(t, x, \frac{x_{2}}{\varepsilon}\right),
\end{aligned}
$$

where $\left(u_{n, k}\right)_{n \in \mathbb{N}, k \in \mathscr{I}_{o s}} \in \mathbb{P}_{o s, \leq}\left(\right.$ resp. $\left.\mathbb{P}_{o s,<}\right)$ for $\beta \leq 1$ (resp. $\beta>1$ ), and for all $n \in \mathbb{N}, \forall k \in \mathfrak{R}_{1}$ (resp. $\mathfrak{R}_{2}$ ), $U_{n, k, 1}$ (resp. $\left.U_{n, k, 2}\right)$ is in $\mathbb{P}_{e v, 1}$ (resp. $\mathbb{P}_{e v, 2}$ ).

Plugging the ansatz (51) into the evolution equation of (47) leads us to the following cascade of equations in the interior :

$$
\begin{cases}\mathscr{L}\left(d \varphi_{k}\right) u_{0, k}=0, & \forall k \in \mathscr{I}_{o s}, \\ i \mathscr{L}\left(d \varphi_{k}\right) u_{n+1, k}+L(\partial) u_{n, k}=0, & \forall n \in \mathbb{N}, \forall k \in \mathscr{I}_{o s}, \\ L_{k}\left(\partial_{X_{1}}\right) U_{0, k, 1}=0, & \forall k \in \mathfrak{R}_{1}, \\ L_{k}\left(\partial_{X_{1}}\right) U_{n+1, k, 1}+L(\partial) U_{n, k, 1}=0, & \forall n \in \mathbb{N}, \forall k \in \mathfrak{R}_{1}, \\ L_{k}\left(\partial_{X_{2}}\right) U_{0, k, 2}=0, & \forall k \in \mathfrak{R}_{2}, \\ L_{k}\left(\partial_{X_{2}}\right) U_{n+1, k, 2}+L(\partial) U_{n, k, 2}=0, & \forall n \in \mathbb{N}, \forall k \in \mathfrak{R}_{2},\end{cases}
$$

where the operators of differentiation in the fast variables $L_{k}\left(\partial_{X_{1}}\right)$ and $L_{k}\left(\partial_{X_{2}}\right)$ are defined by:

$$
\begin{aligned}
& L_{k}\left(\partial_{X_{1}}\right):=A_{1}\left(\partial_{X_{1}}-\mathscr{A}_{1}\left(\underline{\tau}, \xi_{2}^{k}\right)\right) \text { for } k \in \mathfrak{R}_{1}, \\
& L_{k}\left(\partial_{X_{2}}\right):=A_{2}\left(\partial_{X_{2}}-\mathscr{A}_{2}\left(\underline{\tau}, \xi_{1}^{k}\right)\right) \text { for } k \in \mathfrak{R}_{2} .
\end{aligned}
$$


Then, plugging the ansatz (51) in the boundary conditions on $\partial \Omega_{1}$ and on $\partial \Omega_{2}$ gives:

$$
\begin{cases}B_{1}\left[\sum_{j \in \Phi\left(n_{1}\right)} u_{n, j}\right]_{\mid x_{1}=0}=\delta_{n, M+1} g, & \forall n \in \mathbb{N} \\ B_{1}\left[\sum_{j \in \Phi(k) \cap \mathscr{I}_{o s}} u_{n, j}+U_{n, k, 1_{\mid X_{1}=0}}\right]_{\mid x_{1}=0}=0, & \forall n \in \mathbb{N}, \forall k \in\left(\mathscr{R}_{1} \backslash\left\{n_{1}\right\}\right) \cap \mathfrak{R}_{1}, \\ B_{1}\left[\sum_{j \in \Phi(k)} u_{n, j}\right]_{\mid x_{1}=0}=0, & \forall n \in \mathbb{N}, \forall k \notin\left(\mathscr{R}_{1} \backslash\left\{n_{1}\right\}\right) \cap \mathfrak{R}_{1}, \\ B_{2}\left[\sum_{j \in \Psi(k) \cap \mathscr{I}_{o s}} u_{n, j}+U_{n, k, 2_{\mid X_{2}=0}}\right]_{\mid x_{2}=0}=0, & \forall n \in \mathbb{N}, \forall k \in \mathfrak{R}_{2}, \\ B_{2}\left[\sum_{j \in \Psi(k)} u_{n, j}\right]_{\mid x_{2}=0}=0, & \forall n \in \mathbb{N}, \forall k \in \mathscr{R}_{2} \backslash \mathfrak{R}_{2},\end{cases}
$$

where $\delta_{n, p}$ denotes the Kronecker symbol.

Finally, plugging the ansatz (51) into the initial condition of (47), gives:

$$
\forall n \in \mathbb{N}, \begin{cases}u_{n, k_{\mid t=0}}=0, & \forall k \in \mathscr{I}_{o s}, \\ U_{n, k, 1_{\mid t=0}}=0, & \forall k \in \mathfrak{R}_{1}, \\ U_{n, k, 2_{\mid t=0}}=0, & \forall k \in \mathfrak{R}_{2} .\end{cases}
$$

Thanks to the cascades of equations (52)-(55) and (56) we are now able to state our main result about the construction of the geometric optics expansions:

THEOREM 28. Let $T>0$ and assume that the corner problem (47) satisfies Assumptions 1-2-21 and 22.

$\diamond$ If $\beta>1$, then for all $M \in \mathbb{N}$ the corner problem (47) admits a WKB expansion under the form (51).

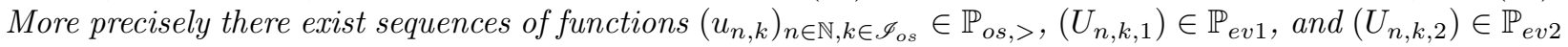
satisfying the cascades of equations (52)-(55) and (56). Moreover one can always choose $g$ in such a way that the leading order in (51) is not identically zero.

$\diamond$ If $\beta \leq 1$, assume that $T<T_{\max }$ if $\beta<1$, then for all $M \in \mathbb{N}$ the corner problem (47) admits a WKB

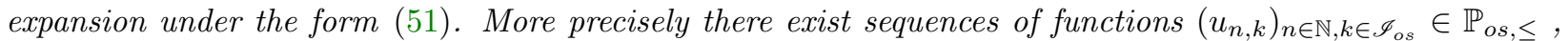
$\left(U_{n, k, 1}\right) \in \mathbb{P}_{\text {ev1 }}$, and $\left(U_{n, k, 2}\right) \in \mathbb{P}_{\text {ev2 }}$ satisfying the cascades of equations (52)-(55) and (56). Moreover one can always choose $g$ in such a way that the leading order in (51) is not identically zero.

The question is now to solve the cascades of equations (52)-(55) and (56). More precisely, to solve the cascades, we are looking for an order of resolution of the different equations and an equation which can be solved before all the others in view to initialize the resolution.

In [4] it is shown that to construct any amplitude in one of the "trees" (that is the sets composing the partition (45) of $\mathscr{I}$ depicted in Figure 2) it is in fact sufficient, thanks to the uniqueness of the type $V$ sequence linking any index of the "tree" to its root (see Proposition 23) and the uniform Kreiss-Lopatinskii condition, to know the amplitude associated to the root. This is also the case here because this determination only uses the uniform Kreiss-Lopatinskii condition which holds outside of the loop. Then it is shown that to know the amplitudes associated to the roots it is sufficient to know the amplitudes associated to the loop's indices. Thus the order of resolution will be exactly the same as in [4]. And also has in [4] the first amplitudes that should be determined are the loop's amplitudes. So we need an new aplitude equation to determine the loop's amplitudes and to initialize the resolution of the cascades of equations.

The first equation of the cascade (52) implies that we have the well-known polarization condition for the oscillating amplitudes $u_{0, k}$, in particular for $k=n_{1}$, we have $u_{0, n_{1}} \in \operatorname{ker} \mathscr{L}\left(d \varphi_{n_{1}}\right)$, in other words we can write:

$$
P_{1}^{n_{1}} u_{0, n_{1}}=u_{0, n_{1}} .
$$

But, thanks to Assumption 1, $\operatorname{ker} \mathscr{L}\left(d \varphi_{n_{1}}\right)$ is one dimensional. Assumption 22 then permits to write:

$$
u_{0, n_{1}}(t, x)=\nu_{0, n_{1}}(t, x) e
$$

for some unknown scalar function $\nu_{0, n_{1}}$ and where $e$ is the vector defined in $12 .{ }^{6}$ For other indices $k \in \mathscr{I}_{\text {os }}$

\footnotetext{
${ }^{6}$ Let us remark that (58) is not true anymore if the operator $L(\partial)$ is hyperbolic with constant multiplicity. In that framework,
} 


$$
u_{0, k}=\nu_{0, k} e_{k},
$$

where $\nu_{0, k}$ is a scalar function and where $e_{k}$ is a generator of $\operatorname{ker} \mathscr{L}\left(d \varphi_{k}\right)$.

More generally for any $n$ let us intoduce the notation:

$$
P_{1}^{n_{1}} u_{n, n_{1}}(t, x)=\nu_{n}(t, x) e,
$$

where $e$ is defined in Definition 12 .

Next let us study the second equation of the cascade (52) written for $n=0$ and $k=n_{1}$. If we compose this equation by $Q_{1}^{n_{1}}$ and use the fact that $\operatorname{ker} Q_{1}^{n_{1}}=\operatorname{Ran} \mathscr{L}\left(d \varphi_{n_{1}}\right)$ thanks to the polarization condition (57) we obtain that $u_{0, n_{1}}$ satisfies the equation :

$$
Q_{1}^{n_{1}} L(\partial) P_{1}^{n_{1}} u_{0, n_{1}}=0 .
$$

From Lax's lemma [11], we have:

$$
Q_{1}^{n_{1}} L(\partial) P_{1}^{n_{1}} u_{0, n_{1}}=\left(\partial_{t}+v_{n_{1}} \cdot \nabla_{x}\right) \nu_{0, n_{1}}=0,
$$

where we used the fact that $Q_{1}^{n_{1}}$ induces an isomorphism from $\operatorname{Ran} P_{1}^{n_{1}}$ to $\operatorname{Ran} Q_{1}^{n_{1}}$. Using the fact that the group velocity $v_{n_{1}}$ is incoming-outgoing, to solve equation (61) we only need to determine the trace of $\nu_{0, n_{1}}$ on $\left\{x_{1}=0\right\}$.

However, the degeneracy of the uniform Kreiss-Lopatinskii condition prevents to determine this trace by the easy classical way. Indeed because without the uniform Kreiss-Lopatinskii condition we can not invert the matrix $B_{1}$ upon the stable subspace $\operatorname{ker} \mathscr{L}\left(d \varphi_{1}\right)$ to recover the value of this trace. The only equation where the trace of $\nu_{0, n_{1}}$ on $\partial \Omega_{1}$ seems to appear is the boundary condition (55) written for $n=0$ that is:

$$
B_{1}\left[\sum_{j \in \Phi\left(n_{1}\right)} u_{0, j}\right]_{\mid x_{1}=0}=0 .
$$

From (58) and the fact that, by definition $e \in \operatorname{ker} B_{1}$, the boundary condition reads:

$$
B_{1}\left[\sum_{j \in \Phi^{*}\left(n_{1}\right)} u_{0, j}\right]_{\mid x_{1}=0}=0,
$$

where we recall that $\Phi^{*}\left(n_{1}\right)$ stands for $\Phi\left(n_{1}\right) \backslash\left\{n_{1}\right\}$. As a consequence this equation does not give any information about $\nu_{0, n_{1 \mid x_{1}=0}}$. So it seems that we shall find another way to determine $\nu_{0, n_{1 \mid x_{1}=0}}$. To do that, we will start by using the method of [8] which has been introduced to construct the geometric optics expansion for the initial boundary value problem belonging to the $W R$ class in the half space and we will see how to adapt this method.

Before to recall the main ideas of the method of determination of $\nu_{0, n_{1 \mid x_{1}=0}}$ by [8], let us remark the following fact. If one assumes that $\nu_{0, n_{1 \mid x_{1}=0}}$ is known and write, once again in view to simplify the notations:

$$
\nu_{0, n_{1 \mid x_{1}=0}}\left(t, x_{2}\right):=\mu_{0}\left(t, x_{2}\right)
$$

(58) has to be remplaced by a decomposition reading :

$$
u_{0, n_{1}}(t, x)=\nu_{0, n_{1}}(t, x) e+\check{v}(t, x),
$$

where $\breve{v}$ lies in some subspace of $E_{1}^{s}\left(i \underline{\tau}, \bar{\xi}_{2}\right)$. We can still apply Kreiss-Lopatinskii condition on this subspace. However, it seems that in that case, one can always determine $\check{v}$ by reiterating the arguments of [4] which lead to ask for the invertibility of an operator reading under the form $(I-\mathbb{T})$. 
then it is easy to determine the others amplitudes $u_{0, k}$, for $k=n_{2}, \ldots, n_{4}$. Indeed, the transport equation determining $\nu_{0, n_{1}}$ reads:

$$
\begin{cases}\left(\partial_{t}+v_{n_{1}} \cdot \nabla_{x}\right) \nu_{0, n_{1}}=0, & \text { on } \Omega_{T} \\ \nu_{0, n_{1 \mid x_{1}}=0}=\mu_{0}, & \text { on } \partial \Omega_{1, T} \\ \nu_{0, n_{1 \mid t \leq 0}}=0, & \text { on } \Omega\end{cases}
$$

Integrating along the characteristics we obtain the explicit formula:

$$
\nu_{0, n_{1}}\left(t, x_{1}, x_{2}\right)=\mu_{0}\left(t-\frac{1}{v_{n_{1}, 1}} x_{1}, x_{2}-\beta_{1}^{-1} x_{1}\right) .
$$

In particular, the trace of $u_{0, n_{1}}$ on $\left\{x_{2}=0\right\}$ is given by

$$
u_{0, n_{1 \mid x_{2}=0}}=\nu_{0, n_{1}}\left(t, x_{1}, 0\right) e,
$$

so, thanks to the uniform Kreiss-Lopatinskii condition for the boundary condition (55) written for $n=0$ and $k=n_{2}$ we are able to determine the trace on $\left\{x_{2}=0\right\}$ of $\nu_{0, n_{2}}$. More precisely, from viii) Proposition 24), the boundary condition for $u_{0, n_{2}}$ reads:

$$
B_{2}\left[\sum_{j \in\left(\Psi\left(n_{2}\right) \cap \mathscr{I}_{o s}\right) \backslash\left\{n_{1}\right\}} u_{0, j}+U_{0, n_{2}, 2_{\mid X_{2}=0}}\right]_{\mid x_{2}=0}=-B_{2} u_{0, n_{1} \mid x_{2}=0}, \quad \text { if } n_{2} \in \mathfrak{R}_{2},
$$

and

$$
B_{2}\left[\sum_{j \in\left(\Psi\left(n_{2}\right) \cap \mathscr{J}_{o s}\right) \backslash\left\{n_{1}\right\}} u_{0, j}\right]_{\mid x_{2}=0}=-B_{2} u_{0, n_{1} \mid x_{2}=0}, \quad \text { if } n_{2} \in \mathscr{R}_{2} \backslash \mathfrak{R}_{2},
$$

Note that in both case the vector in the left hand side is an element of $E_{2}^{s}\left(i \tau, \underline{\xi}_{1}\right)$ so we can apply the uniform Kreiss-Lopatinskii condition. Recall that the inverse of $B_{2}$ on $E_{2}^{s}\left(i \tau, \underline{\xi}_{1}\right)$ is denoted by $\phi_{2}^{n_{2}}$, we apply this inverse and obtain:

$$
\left[\sum_{j \in\left(\Psi\left(n_{2}\right) \cap \mathscr{I}_{o s}\right) \backslash\left\{n_{1}\right\}} u_{0, j}+U_{0, n_{2}, 2_{\mid X_{2}=0}}\right]_{\mid x_{2}=0}=-\phi_{2}^{n_{2}} B_{2} u_{0, n_{1} \mid x_{2}=0}, \quad \text { if } n_{2} \in \mathfrak{R}_{2},
$$

and

$$
\left[\sum_{j \in\left(\Psi\left(n_{2}\right) \cap \mathscr{I}_{o s}\right) \backslash\left\{n_{1}\right\}} u_{0, j}\right]_{\mid x_{2}=0}=-\phi_{2}^{n_{2}} B_{2} u_{0, n_{1} \mid x_{2}=0}, \quad \text { if } n_{2} \in \mathscr{R}_{2} \backslash \mathfrak{R}_{2} .
$$

We then apply $P_{2}^{n_{2}}$ and recall the value of $u_{0, n_{1} \mid x_{2}=0}$ to obtain that in both case:

$$
u_{0, n_{2 \mid x_{2}=0}}=\nu_{0, n_{2 \mid x_{2}=0}} e_{2}=-\nu_{0, n_{1 \mid x_{2}=0}} P_{2}^{n_{2}} \phi_{2}^{n_{2}} B_{2} e .
$$

We then write the vector in the right hand side of the previous equation as:

$$
P_{2}^{n_{2}} \phi_{2}^{n_{2}} B_{2} e=\mathscr{S}^{n_{2}} e_{2}
$$

$$
\begin{cases}\left(\partial_{t}+v_{n_{2}} \cdot \nabla_{x}\right) \nu_{0, n_{2}}=0, & \text { on } \Omega_{T} \\ \nu_{0, n_{2 \mid x_{2}}=0}=-\mathscr{S}^{n_{2}} \mu_{0}, & \text { on } \partial \Omega_{2, T} \\ \nu_{0, n_{1 \mid t \leq 0}}=0, & \text { on } \Omega\end{cases}
$$

$$
\nu_{0, n_{2}}\left(t, x_{1}, x_{2}\right)=-\mu_{0}\left(\mathbf{t}_{2}\left(t, x_{1}, x_{2}\right), \mathbf{x}_{2}\left(x_{1}, x_{2}\right)\right) \mathscr{S}_{n_{2}},
$$


where

$$
\mathbf{t}_{2}\left(t, x_{1}, x_{2}\right):=t-\frac{1}{v_{n_{1}, 1}} x_{1}-\left[\frac{1}{v_{n_{2}, 2}}-\frac{\beta_{2}^{-1}}{v_{n_{1}, 1}}\right] x_{2}, \mathbf{x}_{2}\left(x_{1}, x_{2}\right):=-\beta_{1}^{-1}\left(x_{1}-\beta_{2}^{-1} x_{2}\right) .
$$

We can repeat exactly the same reasoning, applied to the amplitude $u_{0, n_{3}}$ and then to the amplitude $u_{0, n_{4}}$. We thus obtain their values in terms of the unknown trace $\mu_{0}$. More precisely we have:

$$
\nu_{0, n_{3}}\left(t, x_{1}, x_{2}\right)=\mu_{0}\left(\mathbf{t}_{3}\left(t, x_{1}, x_{2}\right), \mathbf{x}_{3}\left(x_{1}, x_{2}\right)\right) \mathscr{S}_{n_{3}},
$$

$$
\nu_{0, n_{4}}\left(t, x_{1}, x_{2}\right)=-\mu_{0}\left(\mathbf{t}_{4}\left(t, x_{1}, x_{2}\right), \mathbf{x}_{4}\left(x_{1}, x_{2}\right)\right) \mathscr{S}_{n_{4}},
$$

where we set for $\nu_{0, n_{3}}$ :

$$
\mathbf{t}_{3}\left(t, x_{1}, x_{2}\right):=t-A x_{1}-\left[\frac{1}{v_{n_{2}, 2}}-\frac{\beta_{2}^{-1}}{v_{n_{1}, 1}}\right] x_{2}, \text { and }, \mathbf{x}_{3}\left(x_{1}, x_{2}\right):=\prod_{j=1}^{2} \beta_{j}^{-1}\left(x_{2}-\beta_{3}^{-1} x_{1}\right),
$$

with $A$ a non-meaningful parameter introduced to simplify the notations. It is precisely given by:

$$
A:=\frac{1}{v_{n_{3}, 1}}-\beta_{3}^{-1}\left(\frac{1}{v_{n_{2}, 2}}-\frac{\beta_{2}^{-1}}{v_{n_{1}, 1}}\right) .
$$

And for $\nu_{0, n_{4}}$ :

$$
\mathscr{S}^{n_{4}}:=\mathbb{S}^{n_{4}} \mathscr{S}^{n_{3}}
$$

$$
\mathbf{t}_{4}\left(t, x_{1}, x_{2}\right):=t-A x_{1}-\left[\frac{1}{v_{n_{4}, 2}}-\beta_{4}^{-1} A\right] x_{2}, \text { and }, \mathbf{x}_{4}\left(x_{1}, x_{2}\right):=-\prod_{j=1}^{3} \beta_{j}^{-1}\left(x_{1}-\beta_{4}^{-1} x_{2}\right) .
$$

And finally where the scalars $\mathbb{S}^{n_{3}}$ and $\mathbb{S}^{n_{4}}$ appearing in (72) and (75) are defined by the relations:

$$
P_{1}^{n_{3}} \phi_{1}^{n_{3}} B_{1} e_{2}:=\mathbb{S}^{n_{3}} e_{3} \text {, and } P_{2}^{n_{4}} \phi_{2}^{n_{4}} B_{2} e_{3}:=\mathbb{S}^{n_{4}} e_{4} .
$$

An important observation for what follows is to remark that the trace $\nu_{0, n_{4} \mid x_{1}=0}$ depends on the particular values, $\mathbf{t}_{4}\left(t, 0, x_{2}\right)$ and $\mathbf{x}_{4}\left(0, x_{2}\right)$. An easy computation shows that the constant in front of $x_{2}$ in $\mathbf{t}_{4}\left(t, 0, x_{2}\right)$ can in fact be expressed in terms of the parameters $\alpha$ and $\beta$, introduced in (49) and (50) and which encode the time needed to make one complete circuit around the loop. More precisely, we have:

$$
\underline{\mathbf{t}}\left(t, x_{2}\right):=\mathbf{t}_{4}\left(t, 0, x_{2}\right)=t-\alpha \beta^{-1} x_{2} \text {, and, } \underline{\mathbf{x}}\left(x_{2}\right):=\mathbf{x}_{4}\left(0, x_{2}\right)=\beta^{-1} x_{2} .
$$

Let us also notice, because it will be important in paragraph 7.1.5, that the knowledge of $u_{0, n_{4}}$ allows us to express all the amplitudes in $\Phi^{*}\left(n_{1}\right)$ in terms of $\mu_{0}$. Indeed, Proposition 17 implies that these amplitudes (except $n_{4}$ ) are in $\mathscr{I}_{i i} \cup \mathscr{I}_{i o}$. So, let $\underline{i} \in \Phi^{*}\left(n_{1}\right) \backslash\left\{n_{4}\right\}$ to determine $u_{0, i}$ for $\underline{i} \in \mathscr{I}_{i o}$ (resp. $\underline{i} \in \mathscr{I}_{i i}$ ) we have to solve the transport equation:

$$
\begin{gathered}
\begin{cases}\left(\partial_{t}+v_{\underline{i}} \cdot \nabla_{x}\right) u_{0, \underline{i}}=0, \\
B_{1}\left[\sum_{j \in \Phi\left(n_{1}\right) \backslash\left\{n_{4}\right\}} u_{0, j}\right]_{\mid x_{1}=0}=-B_{1} u_{0, n_{4} \mid x_{1}=0}, & \text { on } \partial \Omega_{1, T}, \\
u_{0, \underline{\underline{i}} \mid t \leq 0}=0, & \text { on } \Omega,\end{cases} \\
\text { resp. }\left\{\begin{array}{ll}
\left(\partial_{t}+v_{\underline{i}} \cdot \nabla_{x}\right) u_{0, \underline{i}}=0, & \text { on } \Omega_{T}, \\
B_{1}\left[\sum_{j \in \Phi\left(n_{1}\right) \backslash\left\{n_{4}\right\}} u_{0, j}\right]_{\mid x_{1}=0}=-B_{1} u_{0, n_{4 \mid x_{1}=0},}, & \text { on } \partial \Omega_{1, T}, \\
B_{2}\left[\sum_{j \in \Psi\left(n_{2}\right)} u_{0, j}\right]_{\mid x_{2}=0}=0, & \text { on } \partial \Omega_{2, T} \\
u_{0, \underline{i}_{\mid t \leq 0}}=0, & \text { on } \Omega .
\end{array}\right)
\end{gathered}
$$


However, by assumption, the index $\underline{i}$ is associated with a frequency for which the uniform Kreiss-Lopatinskii condition holds, so the boundary condition(s) of (78) (resp.(78)-(79)) can be written under the form:

$$
u_{0, \underline{i}_{\mid x_{1}=0}}=-P_{1}^{\underline{i}} \phi_{1}^{\underline{i}} B_{1} u_{0, n_{4 \mid x_{1}=0}},\left(\operatorname{resp} .\left\{\begin{array}{l}
u_{0, \underline{i}_{\mid x_{1}=0}}=-P_{1}^{\underline{i}} \phi_{1}^{\underline{i}} B_{1} u_{0, n_{4 \mid x_{1}=0}}, \\
u_{0, \underline{i}_{\mid x_{2}=0}=0 .}
\end{array}\right)\right.
$$

Using the expression of $u_{0, n_{4 \mid x_{1}=0}}$ and integrating along the characteristics we obtain the explicit expression:

$$
u_{0, \underline{i}}(t, x)=\mu_{0}\left(\underline{\mathbf{t}}-\frac{1}{v_{\underline{i}, 1}} x_{1}, \underline{\mathbf{x}}-\frac{v_{\underline{i}, 2}}{v_{\underline{i}, 1}} x_{1}\right) \mathscr{S}^{n_{4}} P_{1}^{\underline{i}} \phi_{1}^{\underline{i}} B_{1} e_{4} .
$$

7.1. Initialization of the resolution, determination of the loop's indices . As already mentioned in the previous paragraph to determine $\mu_{0}$ we will adapt the method of [8] from the half space to the quarter space geometry.

We study the boundary condition for the amplitude $u_{1, n_{1}}$ that is (55) written for $n=1$. This is a natural choice because it is the only equation involoving $u_{0, n_{1 \mid x_{1}=0}}$. This boundary condition reads (recall that $M \geq 1)$ :

$$
B_{1}\left[\sum_{j \in \Phi\left(n_{1}\right) \backslash\left\{n_{4}\right\}} P_{1}^{j} u_{1, j}\right]_{\mid x_{1}=0}=-B_{1} u_{1, n_{4}}-B_{1}\left[\sum_{j \in \Phi\left(n_{1}\right) \backslash\left\{n_{4}\right\}}\left(I-P_{1}^{j}\right) u_{1, j}\right]_{\mid x_{1}=0} .
$$

Thanks to Proposition 17, we know that $\Phi\left(n_{1}\right) \backslash\left\{n_{4}\right\}$ is included in $\mathscr{I}_{i o} \cup \mathscr{I}_{i i}$, so the left hand side term is in $B_{1} E_{1}^{s}\left(i \underline{\tau}, \bar{\xi}_{2}\right)$. By definition of $b$ (see Definition 12) multiply by $b$ in the left makes this term vanish. We thus have:

$$
b \cdot B_{1}\left[\sum_{j \in \Phi\left(n_{1}\right) \backslash\left\{n_{4}\right\}}\left(I-P_{1}^{j}\right) u_{1, j}\right]_{\mid x_{1}=0}=-b \cdot B_{1} u_{1, n_{4}} .
$$

To make this equation more explicit in terms of $\mu_{0}$, we use the cascade of equation (52) written for $n=0$. Composing by the partial inverse $R_{1}^{k}$ defined in Definition 25, leads us to the relation:

$$
\forall j \in \Phi\left(n_{1}\right) \backslash\left\{n_{4}\right\},\left(I-P_{1}^{j}\right) u_{1, j}=i R_{1}^{j} L(\partial) u_{0, j}=i R_{1}^{j} L(\partial) P_{1}^{j} u_{0, j},
$$

where we used the polarization condition for $u_{0, j}$ in the right hand side. We thus can write (82) under the form :

$$
b \cdot B_{1}\left[\sum_{j \in \Phi\left(n_{1}\right) \backslash\left\{n_{4}\right\}} R_{1}^{j} L(\partial) P_{1}^{j} u_{0, j}\right]_{\mid x_{1}=0}=i b \cdot B_{1} u_{1, n_{4} \mid x_{1}=0} .
$$

Let us recall the following proposition due to [8] :

Proposition 29 ([8], Proposition 2). For all $j \in \Phi\left(n_{1}\right) \backslash\left\{n_{4}\right\}$, and for all $k=1,2$ let $P_{k}^{j}$, $Q_{k}^{j}$ and $R_{k}^{j}$ be defined in Definition 25 then we have $R_{k}^{j} A_{k} P_{k}^{j}=0$.

Moreover let $b$ be the vector introduced in Definition 12. Then there exists a nonzero real number $\kappa$ such that the following equality holds:

$$
b \cdot B_{1} \sum_{j \in \Phi\left(n_{1}\right) \backslash\left\{n_{4}\right\}} R_{1}^{j} L(\partial) P_{1}^{j}=\kappa\left(\Theta\left(i \underline{\tau}, \bar{\xi}_{2}\right) \partial_{t}+\partial_{\xi_{2}} \Theta\left(i \underline{\tau}, \bar{\xi}_{2}\right) \partial_{2}\right),
$$

where $\Theta$ is defined in Definition 9. Moreover, $\partial_{t} \Theta\left(i \underline{\tau}, \bar{\xi}_{2}\right)=1$ and $\partial_{\xi_{2}} \Theta\left(i \underline{\tau} \partial_{2}, \bar{\xi}_{2}\right)=-\frac{\underline{\tau}}{\bar{\xi}_{2}}:=\underline{c}$.

Proof. We refer to [8] for a complete proof. We will here just show that we have the equality $\partial_{\xi_{2}} \Theta\left(i \underline{\tau}, \bar{\xi}_{2}\right)=$ $-\frac{\tau}{\bar{\xi}_{2}}$. This equality was already shown in [[8], Lemma 7], but since it is important for our purpose, let us give a proof for the sake of completeness. 
Necessarily $\bar{\xi}_{2} \neq 0$ otherwise the Kreiss-Lopatinskii condition will break down for $\gamma>0$ and it would say that the boundary condition on $\partial \Omega_{1}$ does not satisfy the weak Kreiss-Lopatinskii condition. We then use the fact that $d=2$ and the relation $R_{1}^{j} A_{1} P_{1}^{j}=0$. First we have :

$$
\forall j \in \Phi\left(n_{1}\right) \backslash\left\{n_{4}\right\}, \mathscr{L}\left(d \varphi_{j}\right) P_{1}^{j}=\underline{\tau} P_{1}^{j}+\xi_{1}^{j} A_{1} P_{1}^{j}+\bar{\xi}_{2} A_{2} P_{1}^{j}=0,
$$

we compose by $R_{1}^{j}$, the term $R_{1}^{j} A_{1} P_{1}^{j}$ desappears. Then we sum over $j \in \Phi\left(n_{1}\right) \backslash\left\{n_{4}\right\}$ and multiply by $b \cdot B_{1}$ to obtain :

$$
b \cdot B_{1} \sum_{j \in \Phi\left(n_{1}\right) \backslash\left\{n_{4}\right\}} R_{1}^{j} A_{2} P_{1}^{j}=-\frac{\bar{\tau}}{\bar{\xi}_{2}} b \cdot B_{1} \sum_{j \in \Phi\left(n_{1}\right) \backslash\left\{n_{4}\right\}} R_{1}^{j} P_{1}^{j} .
$$

A consequence of this proposition is to recover the transport phenomenon along the boundary already observed in [8]. Moreover thanks to Proposition 29, the equation (83) can be expressed under the form:

$$
\left(\partial_{t} \mu_{0}-\frac{\tau}{\bar{\xi}_{2}} \mu_{0}\right)=\frac{i}{\kappa}\left(b \cdot B_{1} P_{2}^{n_{4}} u_{1, n_{4 \mid x_{1}=0}}+b \cdot B_{1}\left(I-P_{2}^{n_{4}}\right) u_{1, n_{4 \mid x_{1}=0}}\right) .
$$

Thus to determine the unknown trace $\mu_{0}$, we want to solve the same transport equation as in [8]. But, in the analysis of Coulombel and Guès the amplitude $u_{1, n_{4}}$ which acts like a source term in (82) could be determinated regardless $\mu_{0}$. Indeed, for the geometry of the half space $u_{1, n_{4}}$ is an outgoing amplitude so it satisfies a transport equation which does not require any boundary condition for its resolution (see [8] for more details). As a consequence it can be determined just by integration along the characteristics of the source term in the interior and the initial data (and is even zero if these terms vanish).

For the quarter space geometry it is not true anymore because $u_{1, n_{4}}$ depends on $u_{1, n_{3}}$, which depends on $u_{1, n_{2}}$ and so on. However, equation (82) meets with the intuiton that we gave in paragraph 4 because this equation says that the amplification of order zero is "turned on" by the outgoing for the side $\partial \Omega_{1}$ (but incoming for the side $\left.\partial \Omega_{2}\right)$ mode $u_{1, n_{4}}$. Moreover, we mentioned at the end of the previous paragraph that amplitudes $u_{0, j}$ for $j=n_{1}, \ldots n_{4}$ can be expressed in terms of the unknown trace $\mu_{0}$.

So our purpose is now to express the amplitude $u_{1, n_{4}}$ (which acts like a source term in (85)) in terms of the unknow trace $\mu_{0}$. In view to do this, we will show in a first time that the unpolarized part $\left(I-P_{2}^{n_{4}}\right) u_{1, n_{4}}$ can be expressed in terms of $\mu_{0}$. In a second time we will show that the polarized part $P_{2}^{n_{4}} u_{1, n_{4}}$ can be expressed in terms of $\mu_{0}$ and a new unknown trace $\mu_{1}$ (which is just the unknown part of the polarized part of the trace of $u_{1, n_{1}}$ on the side $\left.\partial \Omega_{1}\right)$. The determination of the dependency on $\left(I-P_{2}^{n_{4}}\right) u_{1, n_{4}}$ in terms of $\mu_{0}$ is made in the following paragraph.

7.1.1. Unpolarized part of the terms of order one. In a classical way, after composition of the second equation of (52) (written for $n=0$ and $k=n_{j}, j=1, \ldots, 4$ ) by the pseudo-inverse $R^{n_{j}}$ introduced in Definition 25, we obtain that :

$$
\left(I-P_{1}^{n_{j}}\right) u_{1, n_{j}}=i R_{1}^{n_{j}} L(\partial) u_{0, n_{j}} \text {, if } j \text { is odd, }\left(I-P_{2}^{n_{j}}\right) u_{1, n_{j}}=i R_{2}^{n_{j}} L(\partial) u_{0, n_{j}} \text {, if } j \text { is even, }
$$

where we used the fact that by definition $R_{k}^{n_{j}} \mathscr{L}\left(d \varphi_{n_{j}}\right)=I-P_{k}^{n_{j}}$, for $k=1, \ldots, 2$.

We then use the first equality in Proposition 29. Thanks to this lemma we can compute precisely the values of the unpolarized part of the amplitudes of order one. Indeed for $\left(I-P_{1}^{n_{1}}\right) u_{1, n_{1}}$ we thus have:

$$
\begin{aligned}
\left(I-P_{1}^{n_{1}}\right) u_{1, n_{1}} & =-R_{1}^{n_{1}}\left[\partial_{t}+A_{1} \partial_{1}+A_{2} \partial_{2}\right] \mu_{0}\left(t-\frac{1}{v_{n_{1}, 1}} x_{1}, x_{2}-\beta_{1}^{-1} x_{1}\right) e \\
& =-\left[\left(\partial_{t} \mu_{0}\right) R_{1}^{n_{1}} e+\left(\partial_{2} \mu_{0}\right) R_{1}^{n_{1}} A_{2} e\right]\left(t-\frac{1}{v_{n_{1}, 1}} x_{1}, x_{2}-\beta_{1}^{-1} x_{1}\right),
\end{aligned}
$$

where we used the fact that $P_{1}^{n_{1}} e=e$.

But as $e \in \operatorname{ker} \mathscr{L}\left(d \varphi_{n_{1}}\right)$, we have $\left(\underline{\tau}+\underline{\xi}_{1} A_{1}+\bar{\xi}_{2} A_{2}\right) e=0$. We compose by $R_{1}^{n_{1}}$, and we use Proposition 29 to show that:

$$
R_{1}^{n_{1}} A_{2} e=-\frac{\tau}{\bar{\xi}_{2}} R_{1}^{n_{1}} e
$$

This relation permits to reformulate (87) under the form :

$$
\left(I-P_{1}^{n_{1}}\right) u_{1, n_{1}}=-\left[\partial_{t} \mu_{0}-\frac{\tau}{\bar{\xi}_{2}} \partial_{2} \mu_{0}\right]\left(t-\frac{1}{v_{n_{1}, 1}} x_{1}, x_{2}-\beta_{1}^{-1} x_{1}\right) R_{1}^{n_{1}} e .
$$


The fact that one restricts the study to corner problems (1) in only two space dimensions is used in a non trivial way to establish relation (88) which allows us to reformulate (87) under the form (89). We will see in a moment why this reformulation is so important in the proof.

We do not know if the restriction $d=2$ is really necessary, however it has the advantage to make all the following computations much more simpler.

The same computations can be repeated to determine the unpolarized part of the amplitudes $u_{1, n_{j}}$, for $j=2, \ldots, 4$. Unfortunatly, since our aim is to determine the exact value of the trace $u_{1, n_{4} \mid x_{1}=0}$, we also need the exact values of the unpolarized part of the amplitudes $u_{1, n_{j}}$, for $j=2, \ldots, 4$. After some computations, we find:

$$
\begin{aligned}
& \left(I-P_{2}^{n_{2}}\right) u_{1, n_{2}}=\mathscr{S}^{n_{2}}\left[\left(1+\frac{\underline{\tau}}{v_{n_{1}, 1} \underline{\xi}_{1}}\right) \partial_{t} \mu_{0}+\frac{\underline{\tau}}{\underline{\xi}_{1}} \partial_{2} \mu_{0}\right]\left(\mathbf{t}_{2}, \mathbf{x}_{2}\right) R_{2}^{n_{2}} e_{2}, \\
& \left(I-P_{1}^{n_{3}}\right) u_{1, n_{3}}=-\mathscr{S}^{n_{3}}\left[\left(1-\frac{\underline{\tau}}{v_{n_{2}, 2} \underline{\xi}_{2}}\left(1-\frac{v_{n_{2}, 1}}{v_{n_{1}, 1}}\right)\right) \partial_{t} \mu_{0}+\frac{\underline{\tau}}{\underline{\xi}_{2}} \beta_{2}^{-1} \beta_{1}^{-1} \partial_{2} \mu_{0}\right]\left(\mathbf{t}_{3}, \mathbf{x}_{3}\right) R_{1}^{n_{3}} e_{3}, \\
& \left(I-P_{2}^{n_{4}}\right) u_{1, n_{4}}=\mathscr{S}^{n_{4}}\left[\left(1+\frac{\tau}{\bar{\xi}_{1}} A\right) \partial_{t} \mu_{0}+\frac{\underline{\tau}}{\bar{\xi}_{1}} \prod_{j=1}^{3} \beta_{j}^{-1} \partial_{2} \mu_{0}\right]\left(\mathbf{t}_{4}, \mathbf{x}_{4}\right) R_{2}^{n_{4}} e_{4} .
\end{aligned}
$$

At this step of the proof, the term depending on $\left(I-P_{2}^{n_{4}}\right) u_{1, n_{4}}$ appearing in the right hand side of (85) is expressed in terms of the function $\mu_{0}$. So we just have to do the same for the term $P_{2}^{n_{4}} u_{1, n_{4}}$. In paragraph 7.1.3 we will see how the knowledge of the unpolarized part of the amplitudes of order one enables us to determine the polarized part of the amplitudes of order one. However, before to do this determination, it is useful (because it will simplify a lot the computations) to express the unpolarized part of the amplitudes of order one in terms of the transport operator along the boundary. Moreover, as we will see in paragraph 7.1.4, this reformulation will also be essential for the resolution of the initializating equation (85).

7.1.2. Reformulation of equations (90)-(91) and (92). The following lemma which is just an algebraic property based on a simple computation is however fundamental for our analysis. Indeed it will permit to reformulate equation (85) in a much more pleasant and simple form. The fact that we are able to reformulate (85) in a particular form (more precisely under the form $(I-\widetilde{T})\left(\mathscr{T} \mu_{0}\right)$ see (131) for more details) is not anodyne at all when one wants to solve (85) in view to determine $\mu_{0}$. Indeed it will permit to express $\mathscr{T} \mu_{0}$ as a sum of iterations of the transport operator along the boundary corresponding to the number of complete circuits that have been made.

LEMMA 30. If $d=2$, we have the following equalities:

$$
\begin{aligned}
1+\frac{\underline{\tau}}{\underline{\xi}_{1} v_{n_{1}, 1}} & =-\frac{\bar{\xi}_{2}}{\underline{\xi}_{1}} \beta_{1}^{-1}, \\
\left(1-\frac{\underline{\tau}}{v_{n_{2}, 2} \underline{\xi}_{2}}\left(1-\frac{v_{n_{2}, 1}}{v_{n_{1}, 1}}\right)\right) & =\frac{\bar{\xi}_{2}}{\underline{\xi}_{2}} \beta_{1}^{-1} \beta_{2}^{-1}, \\
1+\frac{\tau}{\bar{\xi}_{1}} A & =-\frac{\bar{\xi}_{2}}{\bar{\xi}_{1}} \beta_{1}^{-1} \beta_{2}^{-1} \beta_{3}^{-1},
\end{aligned}
$$

where we recall that the parameter A appearing in (95) is defined in (74).

Proof. We will only demonstrate (95), the proofs of the two other equalities are simpler and follow exactly the same kind of computations.

First let us develop :

$$
\begin{aligned}
1+\frac{\underline{\tau}}{\bar{\xi}_{1}} A & =\frac{1}{\bar{\xi}_{1}}\left[\bar{\xi}_{1}+\frac{\underline{\tau}}{v_{n_{3}, 1}}\left(1-\frac{v_{n_{3}, 2}}{v_{n_{2}, 2}}\left(1-\frac{v_{n_{2}, 1}}{v_{n_{1}, 1}}\right)\right)\right], \\
& =\frac{1}{v_{n_{3}, 1} v_{n_{2}, 2} v_{n_{1}, 1} \bar{\xi}_{1}}\left[v_{n_{3}, 1} v_{n_{2}, 2} v_{n_{1}, 1} \bar{\xi}_{1}+v_{n_{2}, 2} v_{n_{1}, 1} \underline{\tau}-v_{n_{3}, 2} v_{n_{1}, 1}+v_{n_{3}, 2} v_{n_{2}, 1} \tau\right] .
\end{aligned}
$$

We recall that for all $\left(\tau, \xi_{1}, \xi_{2}\right) \in V$, we have

$$
\tau+\lambda\left(\xi_{1}, \xi_{2}\right)=0
$$


Using the fact that $\lambda$ is homogeneous of degree one, Euler's formula implies that:

$$
\tau+\left(\xi_{1}, \xi_{2}\right) \cdot \nabla_{\xi} \lambda\left(\xi_{1}, \xi_{2}\right)=0 .
$$
tively:

In particular, applying (97) to $f^{n_{1}}=\left(\underline{\tau}, \underline{\xi}_{1}, \bar{\xi}_{2}\right), f^{n_{2}}=\left(\underline{\tau}, \underline{\xi}_{1}, \underline{\xi}_{2}\right)$ and $f^{n_{3}}=\left(\underline{\tau}, \bar{\xi}_{1}, \underline{\xi}_{2}\right)$ we obtain respec-

$$
\begin{aligned}
& \underline{\tau}+v_{n_{1}, 1} \underline{\xi}_{1}+v_{n_{1}, 2} \bar{\xi}_{2}=0, \\
& \underline{\tau}+v_{n_{2}, 1} \underline{\xi}_{1}+v_{n_{2}, 2} \underline{\xi}_{2}=0, \\
& \underline{\tau}+v_{n_{3}, 1} \bar{\xi}_{1}+v_{n_{3}, 2} \underline{\xi}_{2}=0 .
\end{aligned}
$$

From (100) we deduce that:

$$
v_{n_{3}, 1} v_{n_{2}, 2} v_{n_{1}, 1} \bar{\xi}_{1}=-v_{n_{2}, 2} v_{n_{1}, 1} \underline{\tau}-v_{n_{2}, 2} v_{n_{1}, 1} v_{n_{3}, 2} \underline{\xi}_{2} .
$$

Using (99) the second term in the right hand side of (101) this equation can be reformulate under the form:

$$
v_{n_{2}, 2} v_{n_{1}, 1} v_{n_{3}, 2} \underline{\xi}_{2}=v_{n_{1}, 1} v_{n_{3}, 2} \underline{\tau}+v_{n_{2}, 1} v_{n_{1}, 1} v_{n_{3}, 2} \underline{\xi}_{1} .
$$

We then use (98) in the second term in the right hand side of (102). We obtain:

$$
v_{n_{2}, 1} v_{n_{1}, 1} v_{n_{3}, 2} \underline{\xi}_{1}=-v_{n_{2}, 1} v_{n_{3}, 2} \underline{\tau}-v_{n_{1}, 2} v_{n_{2}, 1} v_{n_{3}, 2} \bar{\xi}_{2} .
$$

Combining equations (101), (102) and (103) it follows that:

$$
v_{n_{3}, 1} v_{n_{2}, 2} v_{n_{1}, 1} \bar{\xi}_{1}=-v_{n_{2}, 2} v_{n_{1}, 1} \underline{\tau}+v_{n_{3}, 2} v_{n_{1}, 1} \underline{\tau}-v_{n_{3}, 2} v_{n_{2}, 1} \underline{\tau}-v_{n_{1}, 2} v_{n_{2}, 1} v_{n_{3}, 2} \bar{\xi}_{2} .
$$

Equality (95) follows from (96) combined with (104).

For simplicity we introduce the notation:

$$
\mathscr{T}:=\partial_{t}-\frac{\tau}{\bar{\xi}_{2}} \partial_{2}
$$

which is the same transport operator along the boundary as in [8].

Thanks to Lemma 30 formulas (90)-(91) and (92), which give the unpolarized part of the amplitudes $u_{1, n_{i}}$, for $i=2, \ldots, 4$, can be written under the following more pleasant form:

$$
\begin{aligned}
\left(I-P_{2}^{n_{2}}\right) u_{1, n_{2}} & =-\mathscr{S}^{n_{2}} \frac{\bar{\xi}_{2}}{\underline{\xi}_{1}} \beta_{1}^{-1}\left(\mathscr{T} \mu_{0}\right)\left(\mathbf{t}_{2}, \mathbf{x}_{2}\right) R_{2}^{n_{2}} e_{2}, \\
\left(I-P_{1}^{n_{3}}\right) u_{1, n_{3}} & =-\mathscr{S}^{n_{3}} \frac{\bar{\xi}_{2}}{\underline{\xi}_{2}} \prod_{j=1}^{2} \beta_{j}^{-1}\left(\mathscr{T} \mu_{0}\right)\left(\mathbf{t}_{3}, \mathbf{x}_{3}\right) R_{1}^{n_{3}} e_{3}, \\
\left(I-P_{2}^{n_{4}}\right) u_{1, n_{4}} & =-\mathscr{S}^{n_{4}} \frac{\bar{\xi}_{2}}{\bar{\xi}_{1}} \prod_{j=1}^{3} \beta_{j}^{-1}\left(\mathscr{T} \mu_{0}\right)\left(\mathbf{t}_{4}, \mathbf{x}_{4}\right) R_{2}^{n_{4}} e_{4} .
\end{aligned}
$$

We thus remark that the unpolarized part of the amplitudes $u_{1, n_{j}}, j=1, \ldots, 4$ depend of the same transport operator which is precisely the transport operator applied to $\mu_{0}$ in equation (85).

Let us conclude this paragraph by the determination of the unpolarized part of the amplitudes $u_{1, j}$ for $\underline{i} \in \Phi\left(n_{1}\right)^{*} \backslash\left\{n_{4}\right\}$. The knowledge of these amplitudes will be useful in paragraph 7.1.5. From (80) and the relation (86), one easily obtains, that after the reformulation of $\left(I-P_{n_{4}}\right) u_{1, n_{4}}$, we have:

$$
\left(I-P_{1}^{\underline{i}}\right) u_{1, \underline{i}_{\mid x_{1}=0}}=i\left[\mathscr{S}^{n_{4}} R_{1}^{\underline{i}} L(\partial) \mu_{0}\left(\mathbf{t}_{4}, \mathbf{x}_{4}\right) P_{1}^{\underline{i}} \phi_{1}^{\underline{i}} B_{1} e_{4}\right]_{\mid x_{1}=0},
$$

from which we deduce that up to some derivatives and scalar multiplications, $\left(I-P_{1}^{\underline{i}}\right) u_{1, \underline{\underline{i}_{\mid x}=0}}$ depends on $\mu_{0}(\underline{\mathbf{t}}, \underline{\mathbf{x}})$. 
7.1.3. Polarized part of the terms of order one. The knowledge of the unpolarized part of the amplitudes of order one enables us to determine the polarized part of the amplitudes of order one, and will conclude the determination of the right hand side in equation (85).

Indeed, let us consider equation (52) written for $n=1$ and $k=n_{j}, j=1, \ldots 4$. This equation reads:

$$
\begin{aligned}
& i \mathscr{L}\left(d \varphi_{n_{j}}\right) u_{2, n_{j}}+L(\partial) P_{1}^{n_{j}} u_{1, n_{j}}=-L(\partial)\left(I-P_{1}^{n_{j}}\right) u_{1, n_{j}}, \text { for } j \text { odd, } \\
& i \mathscr{L}\left(d \varphi_{n_{j}}\right) u_{2, n_{j}}+L(\partial) P_{2}^{n_{j}} u_{1, n_{j}}=-L(\partial)\left(I-P_{2}^{n_{j}}\right) u_{1, n_{j}} \text {, for } j \text { even. }
\end{aligned}
$$

We compose by $Q_{1}^{n_{1}}, Q_{1}^{n_{3}}$ in the first equation, by $Q_{2}^{n_{2}}, Q_{2}^{n_{4}}$ in the second one. This makes the term depending of $u_{2, n_{j}}$ disappears and from Lax's lemma we obtain that the polarized parts of order one satisfy the transport equations:

$$
\begin{gathered}
\left(\partial_{t}+v_{n_{j}} \cdot \nabla_{x}\right) Q_{1}^{n_{j}} P_{1}^{n_{j}} u_{1, n_{j}}=-Q_{1}^{n_{j}} L(\partial)\left(I-P_{1}^{n_{j}}\right) u_{1, n_{j}}, \text { if } j=1,3, \\
\left(\partial_{t}+v_{n_{j}} \cdot \nabla_{x}\right) Q_{2}^{n_{j}} P_{2}^{n_{j}} u_{1, n_{j}}=-Q_{2}^{n_{j}} L(\partial)\left(I-P_{2}^{n_{j}}\right) u_{1, n_{j}}, \text { if } j=2,4,
\end{gathered}
$$

with initial and boundary conditions given by (55)-(56). A preliminary to obtain the exact values of the solutions of (110)-(111) is thus to determine the source terms. This can be done thanks to the reformulation made in paragraph 7.1.2. Let us start with the term $Q_{1}^{n_{1}} L(\partial)\left(I-P_{1}^{n_{1}}\right) u_{1, n_{1}}$, from equation (89), an explicit computation gives:

$$
\begin{array}{r}
Q_{1}^{n_{1}} L(\partial)\left(I-P_{1}^{n_{1}}\right) u_{1, n_{1}}=-\left[Q_{1}^{n_{1}} R_{1}^{n_{1}} e\left(\partial_{t t}^{2} \mu_{0}-\frac{\tau}{\bar{\xi}_{2}} \partial_{t 2}^{2} \mu_{0}\right)+Q_{1}^{n_{1}} A_{2} R_{1}^{n_{1}} e\left(\partial_{t 2}^{2} \mu_{0}-\frac{\tau}{\bar{\xi}_{2}} \partial_{22}^{2} \mu_{0}\right)\right] \\
\left(t-\frac{1}{v_{n_{1}, 1}} x_{1}, x_{2}-\beta_{1}^{-1} x_{1}\right) .
\end{array}
$$

Where we used the fact that $Q_{1}^{n_{1}} A_{1} R_{1}^{n_{1}} e=0$ (see Proposition 29 for more details).

To make this equation more explicit in terms of $\mu_{0}$, as it has been done for the determination of the unpolarized parts of order one, we are looking for a relation linking $Q_{1}^{n_{1}} R_{1}^{n_{1}} e$ and $Q_{1}^{n_{1}} A_{2} R_{1}^{n_{1}} e$ in view to factorize (112).

We recall that $\operatorname{ker} Q_{1}^{n_{1}}=\operatorname{Ran} \mathscr{L}\left(d \varphi_{n_{1}}\right)$ so for all $X \in \mathbb{C}^{N}$ we have $Q_{1}^{n_{1}} \mathscr{L}\left(d \varphi_{n_{1}}\right) X=0$. In particular, for $X=R_{1}^{n_{1}} e$ we obtain:

$$
\underline{\tau} Q_{1}^{n_{1}} R_{1}^{n_{1}} e+\underline{\xi}_{1} Q_{1}^{n_{1}} A_{1} R_{1}^{n_{1}} e+\bar{\xi}_{2} Q_{1}^{n_{1}} A_{2} R_{1}^{n_{1}} e=0 \Longrightarrow Q_{1}^{n_{1}} A_{2} R_{1}^{n_{1}} e=-\frac{\underline{\tau}}{\bar{\xi}_{2}} Q_{1}^{n_{1}} R_{1}^{n_{1}} e .
$$

Using this relation in (112) gives:

$$
\begin{aligned}
Q_{1}^{n_{1}} L(\partial)\left(I-P_{1}^{n_{1}}\right) u_{1, n_{1}}= & -\left[Q_{1}^{n_{1}} R_{1}^{n_{1}} e\left(\partial_{t t}^{2} \mu_{0}-2 \frac{\frac{\tau}{\bar{\xi}_{2}}}{\partial_{t 2}} \mu_{0}+\left(\frac{\tau}{\bar{\xi}_{2}}\right)^{2} \partial_{22}^{2} \mu_{0}\right)\right] \\
& \left(t-\frac{1}{v_{n_{1}, 1}} x_{1}, x_{2}-\beta_{1}^{-1} x_{1}\right), \\
= & -\left[Q_{1}^{n_{1}} R_{1}^{n_{1}} e \mathscr{T}^{2} \mu_{0}\right]\left(t-\frac{1}{v_{n_{1}, 1}} x_{1}, x_{2}-\beta_{1}^{-1} x_{1}\right),
\end{aligned}
$$

where we recall that the transport operator $\mathscr{T}$ is defined in (105).

Let us remark that, exactly as for the unpolarized part of the amplitudes of order one, the source term in (110) expresses in terms of the transport operator $\mathscr{T}$. However this time (114) involves some power of the operator $\mathscr{T}$.

We then repeat the same kind of computations for the terms $Q_{2}^{n_{2}} L(\partial)\left(I-P_{2}^{n_{2}}\right) u_{1, n_{2}}, Q_{1}^{n_{3}} L(\partial)(I-$ $\left.P_{1}^{n_{3}}\right) u_{1, n_{3}}$ and $Q_{2}^{n_{4}} L(\partial)\left(I-P_{2}^{n_{4}}\right) u_{2, n_{4}}$. Using analogous relations as (113), more precisely:

$$
\begin{aligned}
Q_{2}^{n_{2}} A_{1} R_{2}^{n_{2}} \mathscr{S}^{n_{2}} e & =-\frac{\underline{\tau}}{\xi_{1}} Q_{2}^{n_{2}} R_{2}^{n_{2}} \mathscr{S}^{n_{2}} e, \\
Q_{1}^{n_{3}} A_{2} R_{1}^{n_{3}} \mathscr{S}^{n_{3}} e & =-\frac{\underline{\tau}}{\xi_{2}} Q_{1}^{n_{3}} R_{1}^{n_{3}} \mathscr{S}^{n_{3}} e \\
Q_{2}^{n_{4}} A_{1} R_{2}^{n_{4}} \mathscr{S}^{n_{4}} e & =-\frac{\underline{\tau}}{\underline{\xi}_{1}} Q_{2}^{n_{4}} R_{2}^{n_{4}} \mathscr{S}^{n_{4}} e,
\end{aligned}
$$


1041

1042

1043

1044

1045

1046

1047

1048

1049

1050

1051

1052

1053

1054

1055

1056

1057

1058

1059

1060

1061

1062

1063

1064

1065

1066

1067

1068

1069

1070

1071

1072

1073

1074

1075

and Lemma 30 in equations (106)-(107) and (108), some tedious (but explicit) computations give:

$$
\begin{aligned}
& Q_{2}^{n_{2}} L(\partial)\left(I-P_{2}^{n_{2}}\right) u_{1, n_{2}}=\mathscr{S}^{n_{2}} \beta_{1}^{-2}\left(\frac{\bar{\xi}_{2}}{\underline{\xi}_{1}}\right)^{2}\left(\mathscr{T}^{2} \mu_{0}\right)\left(\mathbf{t}_{2}, \mathbf{x}_{2}\right)_{2}^{n_{2}} R_{2}^{n_{2}} e_{2}, \\
& Q_{1}^{n_{3}} L(\partial)\left(I-P_{1}^{n_{3}}\right) u_{1, n_{3}}=-\mathscr{S}^{n_{3}} \beta_{1}^{-2} \beta_{2}^{-2}\left(\frac{\bar{\xi}_{2}}{\xi_{2}}\right)^{2}\left(\mathscr{T}^{2} \mu_{0}\right)\left(\mathbf{t}_{3}, \mathbf{x}_{3}\right) Q_{1}^{n_{3}} R_{1}^{n_{3}} e_{3}, \\
& Q_{2}^{n_{4}} L(\partial)\left(I-P_{2}^{n_{4}}\right) u_{1, n_{4}}=\mathscr{S}^{n_{4}} \prod_{j=1}^{3} \beta_{j}^{-2}\left(\frac{\bar{\xi}_{2}}{\bar{\xi}_{1}}\right)^{2}\left(\mathscr{T}^{2} \mu_{0}\right)\left(\mathbf{t}_{4}, \mathbf{x}_{4}\right) Q_{2}^{n_{4}} R_{2}^{n_{4}} e_{4} .
\end{aligned}
$$

More details about those computations can be find in Appendix 9.2.

Now that the source term in equation (110) is express in terms of $\mathscr{T}^{2} \mu_{0}$, we can solve this equation in terms of $\mathscr{T}^{2} \mu_{0}$. From the strict dissipativity of $(1)$, we have $\operatorname{ker} B_{1}=E_{1}^{s}\left(i \underline{\tau}, \bar{\xi}_{2}\right)=$ vect $\{e\}$. So we write as in the beginning of paragraph 7.1 :

$$
P_{1}^{n_{1}} u_{1, n_{1}}\left(t, x_{2}\right):=\nu_{1, n_{1}}\left(t, x_{2}\right) e=\mu_{1}\left(t, x_{2}\right) e .
$$

The transport equation (110) becomes :

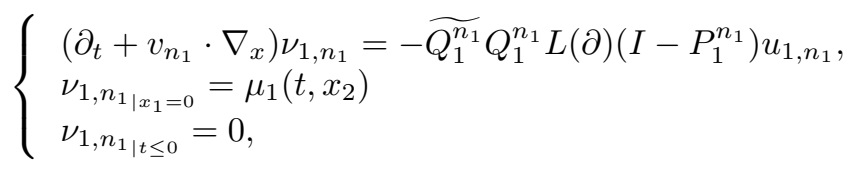

where $\widetilde{Q_{1}^{n_{1}}}$ denotes the inverse of the restriction of $Q_{1}^{n_{1}}$ to $\operatorname{Ran} P_{1}^{n_{1}}$ and where the trace function $\mu_{1}$ is an unknown. Then integrating along the characteristics gives the exact value of $\nu_{1, n_{1}}$ (and thus also of $P_{1}^{n_{1}} u_{1, n_{1}}$ ) in terms of the unknown traces $\mu_{0}, \mu_{1}$. More precisely, we have to study two separates cases:

- $t-\frac{1}{v_{n_{1}, 1} x_{1}}<0$. Then the transported information is above the characteristic, the transported condition is the initial one. Moreover, as the function $\mu_{0}$ is assumed to satisfy $\mu_{0_{\mid t \leq 0}}$, one can check on (114) that the transport associated to the source term in the interior is zero. Consequently the associated solution is zero.

- $t-\frac{1}{v_{n_{1}, 1} x_{1}}>0$. Then the transported information is below the characteristic, the transported condition is the boundary condition and this time the transport associated to the source term in the interior does not necessarily vanish. We Integrate along the characteristics to obtain the explicit value:

$$
\begin{aligned}
\nu_{1, n_{1}} & =\mu_{1}\left(t-\frac{1}{v_{n_{1}, 1}} x_{1}, x_{2}-\beta_{1}^{-1} x_{1}\right) \\
& +c \int_{0}^{x_{1}} \mathscr{T}^{2} \mu_{0}\left(t-\frac{s}{v_{n_{1}, 1}}-\frac{s}{v_{n_{1}, 1}}\left(x_{1}-s\right), x_{2}-\beta_{1}^{-1} s-\beta_{1}^{-1}\left(x_{1}-s\right)\right) d s \\
& =\mu_{1}\left(t-\frac{1}{v_{n_{1}, 1}} x_{1}, x_{2}-\beta_{1}^{-1} x_{1}\right)+c x_{1} \mathscr{T}^{2} \mu_{0}\left(t-\frac{s}{v_{n_{1}, 1}} x_{1}, x_{2}-\beta_{1}^{-1} x_{1}\right),
\end{aligned}
$$

for some explicit constant $c$.

To obtain (123), one has to use the explicit value of the source term given in (114), and check that the source term lies along the characteristics. So the integral term in (123) is just a multiplication by the length of the characteristic.

From this formula, and the formula giving the unpolarized part of $u_{1, n_{1}}$, we finally obtain the following value for $u_{1, n_{1}}$ :

$$
\begin{aligned}
u_{1, n_{1}} & =P_{1}^{n_{1}} u_{1, n_{1}}+\left(I-P_{1}^{n_{1}}\right) u_{1, n_{1}} \\
& =\left[\mu_{1} e+c x_{1} \mathscr{T}^{2} \mu_{0} e-\mathscr{T} \mu_{0} R_{1}^{n_{1}} e\right]\left(t-\frac{1}{v_{n_{1}, 1}} x_{1}, x_{2}-\beta_{1}^{-1} x_{1}\right),
\end{aligned}
$$

from which, we deduce the value of $u_{1, n_{1 \mid x_{2}=0}}$ in terms of $\mathscr{T} \mu_{0}$ and $\mu_{1}$ :

$$
u_{1, n_{1 \mid x_{2}=0}}=\left[\mu_{1} e+c x_{1} \mathscr{T}^{2} \mu_{0} e-\mathscr{T} \mu_{0} R_{1}^{n_{1}} e\right]\left(t-\frac{1}{v_{n_{1}, 1}} x_{1},-\beta_{1}^{-1} x_{1}\right) .
$$


where $c$ stands for an explicitly computable constant.

The knowledge of this trace enable us to determine the amplitude $u_{1, n_{2}}$ (recall that $u_{1, n_{1 \mid x_{2}=0}}$ acts as a boundary source term in the transport equation determining $u_{1, n_{2}}$ ). Thus we can evaluate $u_{1, n_{2} \mid x_{1}=0}$ and then determine the amplitude $u_{1, n_{3}}$. Finally the knowledge of $u_{1, n_{3 \mid x_{2}=0}}$ perits to determine the amplitude $u_{1, n_{4}}$ and more precisely its trace on $\left\{x_{1}=0\right\}$ which appears in equation (85). Indeed, thanks to paragraph 7.1.1, to fully determine the $u_{1, n_{j}}, j=2, \ldots, 4$ it only remains to determine their polarized parts. Let us define:

$$
P_{2}^{n_{j}} u_{1, n_{j}}(t, x)=\nu_{1, n_{j}}(t, x) e_{j} \text {, for } j=2,4 \text { and } P_{1}^{n_{3}} u_{1, n_{3}}(t, x)=\nu_{1, n_{3}}(t, x) e_{3},
$$

then, thanks to the uniform Kreiss-Lopatinskii condition and the fact that $n_{2} \in \mathscr{I}_{o i}, \nu_{1, n_{2}}$ satisfies the transport equation:

$$
\left\{\begin{array}{l}
\left(\partial_{t}+v_{n_{2}} \cdot \nabla_{x}\right) \nu_{1, n_{2}}=-\widetilde{Q_{2}^{n_{2}}} Q_{2}^{n_{2}} L(\partial)\left(I-P_{2}^{n_{2}}\right) u_{1, n_{2}}, \\
\nu_{1, n_{2} \mid x_{2}=0}=-\mathscr{S}^{n_{2}} u_{1, n_{1 \mid x_{2}=0}}-\mathscr{S}^{n_{2}}\left(I-P_{2}^{n_{2}}\right) u_{1, n_{2} \mid x_{2}=0} \\
\nu_{1, n_{2} \mid t \leq 0}=0
\end{array}\right.
$$

where as for $\nu_{1, n_{2}}, \widetilde{Q_{2}^{n_{2}}}$ denotes the inverse of the restriction of $Q_{2}^{n_{2}}$ to $\operatorname{Ran} P_{2}^{n_{2}}$. The source terms in (125) are given by (118), (124) and (106). We integrate along the characteristics, once again there are two cases to separate:

- $t-\frac{1}{v_{n_{2}, 2} x_{2}} \leq 0$. The transported information comes from the initial condition. Noticing that the source term in the interior is evaluated in $\mathbf{t}_{2}(t, x) \leq t-\frac{1}{v_{n_{2}, 2} x_{2}}$ (we recall that $\mathbf{t}_{2}$ is defined in (69)) we deduce that the transport of the source term in the interior is also zero.

- $t-\frac{1}{v_{n_{2}, 2} x_{2}}>0$. The transported information comes from the boundary condition. We integrate along the characteristics. This gives:

$$
\nu_{1, n_{2}}=-\mathscr{S}^{n_{2}}\left[\mu_{1}+c x_{1} \mathscr{T}^{2} \mu_{0} e-\mathscr{T} \mu_{0} R_{1}^{n_{1}} e\right]\left(\mathbf{t}_{2}, \mathbf{x}_{2}\right)+c_{2} x_{2} \mathscr{T}^{2} \mu_{0}\left(\mathbf{t}_{2}, \mathbf{x}_{2}\right)
$$

where $c_{2}:=\beta_{1}^{-2}\left(\frac{\bar{\xi}_{2}}{\underline{\xi}_{1}}\right)^{2} \widetilde{Q_{2}^{n_{2}}} Q_{2}^{n_{2}} R_{2}^{n_{2}} \mathscr{S}^{n_{2}} e$, however this value is not really important in the end of the proof. Once again the multiplication by the factor $x_{2}$ in the last term of the right hand side of (126) comes from the fact that the source term in the interior of (125) lies on the characteristics.

So we now have an explicit formula for the polarized part of the amplitude $u_{n_{2}, 1}$, from which we deduce the value of its trace on $\partial \Omega_{1}$. We then solve the transport equation and determine the trace of $u_{n_{2}, 1}$ on $\partial \Omega_{2}$. Then we use this trace in the transport equation determining $\nu_{1, n_{3}}$. Integrating along the characteristics we obtain the trace of $P_{1}^{n_{3}} u_{n_{3}, 1}$. The important fact is that (as in the resolution of the resolution of (122) and (125)), the source term in the interior already lies along the characteristics so its contribution to the transport phenomenon is just a multiplication by $x_{1}$ of itself. Moreover equations (107) and (119) tell us that the source terms in the transport equation determining $\nu_{1, n_{3}}$ depends on $\mu_{1}, \mathscr{T} \mu_{0}$ and $\mathscr{T}^{2} \mu_{0}$, all evaluated in $\left(\mathbf{t}_{2}, \mathbf{x}_{2}\right)$. As a consequence $P_{1}^{n_{3}} u_{1, n_{3}}$ depends on $\mu_{1}, \mathscr{T} \mu_{0}$ and $\mathscr{T}^{2} \mu_{0}$, all evaluated in $\left(\mathbf{t}_{3}, \mathbf{x}_{3}\right)$, then from equation (107) so do $u_{1, n_{3}}$.

To conclude, we compute the trace of $u_{1, n_{3}}$ on $\partial \Omega_{2}$ and we use it as a source term in the transport equation determining $\nu_{1, n_{4}}$. Repeating exactly the same arguments we show that the $P_{2}^{n_{4}} u_{1, n_{4}}$ depends on $\mu_{1}, \mathscr{T} \mu_{0}$ and $\mathscr{T}^{2} \mu_{0}$, all evaluated in $\left(\mathbf{t}_{4}, \mathbf{x}_{4}\right)$. This implies that $P_{2}^{n_{4}} u_{1, n_{4} \mid x_{1}=0}$ depends on $\mu_{1}, \mathscr{T} \mu_{0}$ and $\mathscr{T}^{2} \mu_{0}$, all evaluated in $\left(\mathbf{t}_{4}, \mathbf{x}_{4}\right)$.

Consequently the right hand side of (85) (because it only involves $P_{2}^{n_{4}} u_{1, n_{4} \mid x_{1}=0}$ and $\left(I-P_{2}^{n_{4}} u_{1, n_{4} \mid x_{1}=0}\right)$ has been expressed in terms of $\mu_{1}, \mathscr{T} \mu_{0}$ and $\mathscr{T}^{2} \mu_{0}$, all evaluated in $(\underline{\mathbf{t}}, \underline{\mathbf{x}})$ (recall the notation $(\underline{\mathbf{t}}, \underline{\mathbf{x}})=\left(\mathbf{t}_{4}, \mathbf{x}_{4}\right)$ ), more precisely (85) reads:

$$
\left(\mathscr{T} \mu_{0}\right)\left(t, x_{2}\right)-\left(\mathbb{T} \mu_{0}\right)\left(t, x_{2}\right)=\frac{i}{\kappa} \mathscr{S}^{n_{4}} e \mu_{1}(\underline{\mathbf{t}}, \underline{\mathbf{x}})
$$

where $\mathbb{T}$ is up to multiplications by some (possibly complicated functions) the sum of $\mathscr{T} \mu_{0}$ and $\mathscr{T}^{2} \mu_{0}$ followed 


\footnotetext{
${ }^{7}$ Notice that the precise values of the constant $c_{1}$ and of the function $c_{2}$ can be exactly expressed from equations (108) and explicit computations in the resolution of the transport equations mentioned in paragraph 7.1.3. However, it is useless for our purpose.

${ }^{8}$ Let us stress that the operator $\mathbb{T}$ of this paper has nothing in common with the operator $\mathbb{T}$ of [4]. Indeed, the operator $\mathbb{T}$ of [4] is of order zero and necessitates to be well-defined the uniform Kreiss-Lopatinskii condition.

where we set $\widetilde{\mu}_{1}:=\frac{i}{\kappa} \mathscr{S}^{n_{4}} e \mu_{1}$ and then drop the tilde and where we did the same operation (up to the sign) for $\widetilde{g}^{8}$.

In the following paragraph, we explain how this particular structure for equation (85) permits us to determine the unknown $\mu_{0}$.

7.1.4. Resolution of equation (85), preliminary study. The resolution of equation (85) is based upon the study of the influence of the change of variables $(\underline{\mathbf{t}}, \underline{\mathbf{x}})$ on the profile spaces. We recall that these spaces are not defined in the same way depending on the value of the dilatation parameter $\beta$. That is why the resolution of equation (85) needs to be discussed in two distinct frameworks.

The case $\beta \leq 1$, the information gets closer of the corner or admits a periodic pattern. Before to solve equation (85) we give a useful property of the behaviour spaces of profiles $\mathscr{P}_{b, \leq}^{M-n}$ compared with the change of variables $(\underline{\mathbf{t}}, \underline{\mathbf{x}})$. More precisely this property states that if a function $\mu$ is one of the $\mathscr{P}_{b, \leq}^{M-n}$, which essentially means that it starts to be non-zero only after a certain time (corresponding to $n$ complete circuits around the loop), then $\mu(\mathbf{t}, \mathbf{x})$ will be non-zero only after the time corresponding to $n+1$ complete circuits. If we use formally this result in (127) this means that the one order unknown trace can only affect the one order unknown trace after one complete circuit. This formally meets the intuition given in Section 4 which claims that the zero order amplitude in the WKB expansion has been turned on by the one order amplitude after it has made one complete circuit around the loop.

Proposition 31. If $\mu \in \mathscr{P}_{b, \leq}^{M-n}$ then $\mu(\underline{\mathbf{t}}, \underline{\mathbf{x}}) \in \mathscr{P}_{b, \leq}^{M-n+1}$.

Proof. We assume that $M-n>0$, the other possible values of $M-n>0$ are treated similarly and will not be demonstrated here.

In a first time we show that $\mu(\underline{\mathbf{t}}, \underline{\mathbf{x}})$ is zero for $t \in]-\infty, \underline{T}_{M-n+1}[$.

Firstly if $t<\underline{T}_{M-n}$ then so do $\underline{\mathbf{t}}<\underline{T}_{M-n}$ and the result is automatic. So we assume that $T_{M-n}<t<$ $\underline{T}_{M-n+1}$ and $\underline{\mathbf{t}}<\underline{T}_{M-n+1}$. By definition of $\mathscr{P}_{b}^{M-n}, \mu(\underline{\mathbf{t}}, x)$ is zero if $x_{2}<\beta^{M-n} \underline{y}_{0}$. Thus we restrict our attention to the case $x_{2} \geq \beta^{M-n} \underline{y}_{0}$. We thus have that:

$$
\underline{\mathbf{t}}<\underline{T}_{M-n+1}-\alpha \beta^{M-n} \underline{y}_{0}=\underline{T}_{M-n},
$$

from which it follows that $\mu(\underline{\mathbf{t}}, \cdot)$ is zero.

Now, let $k$ be such that $M-n+1 \leq k \leq M$ and fix $t \in\left[\underline{T}_{k}, \min \left(\underline{T}_{k+1}, T\right)\right.$ [, we will distinguish three cases depending on the value of $\underline{\mathbf{t}}$ :

i) If $\underline{\mathbf{t}}<\underline{T}_{k-1}$ then it follows that:

$$
\underline{T}_{k}<t<\alpha \beta^{-1} x_{2}+\underline{T}_{k-1},
$$

from which we deduce that $x_{2}>\beta^{k} \underline{y}_{0}$. Consequently it is, in fact, not useful to study this case to show that $\mu(\underline{\mathbf{t}}, \underline{\mathbf{x}})$ is zero for $x_{2}<\beta^{k} \underline{y}_{0}$.

ii) If $\underline{T}_{k-1}<\underline{\mathbf{t}}<\underline{T}_{k}$ then by definition of $\mathscr{P}_{b, \leq}^{M-n}, \mu(\underline{\mathbf{t}}, x)$ is zero for $x_{2}<\beta^{k-1} \underline{y}_{0}$ and as a consequence $\mu(\underline{\mathbf{t}}, \underline{\mathbf{x}})$ is zero for $x_{2}<\beta^{k} \underline{y}_{0}$.

iii) If $\underline{T}_{k}<\underline{\mathbf{t}}$ then we can repeat the argument applied in equation (130) to show that, this time, necessarily we have $\underline{y}_{0} \beta^{k+1}>x_{2}$ for which we deduce that $\mu(\underline{\mathbf{t}}, \underline{\mathbf{x}})$ is zero. 
We also give a useful proposition concerning the influence of the change of variables $(\underline{\mathbf{t}}, \underline{\mathbf{x}})$ on the source term $g$. This proposition shows that even if $g$ is not in some $\mathscr{P}_{b}^{k}$ the change of variable $(\underline{\mathbf{t}}, \underline{\mathbf{x}})$ has good properties on the support of $g$. More precisely this proposition states that the change of variable $(\underline{\mathbf{t}}, \underline{\mathbf{x}})$ has essentially the same influence on $g$ that if was a function in some $\mathscr{P}_{b}^{k}$.

PROPOSITION 32. Let $g$ be a smooth function which is zero for negative times and satisfying

$$
\forall t>0, g(t, x)=0 \text { if } x<\underline{y}_{0} .
$$

Then for all $l \in \mathbb{N}^{*}, g\left(\underline{\mathbf{t}}^{l}, \underline{\mathbf{x}}^{l}\right) \in \mathscr{P}_{b, \leq}^{l}$.

Proof. The proof picks up some ideas from the proof of Proposition 31. However, we give it for the sake of completness.

First let us show that $g\left(\underline{\mathbf{t}}^{l}, \underline{\mathbf{x}}^{l}\right) \in \mathscr{P}_{b, \leq}^{l}$ is zero for $t<\underline{T}_{l}$. If $\underline{\mathbf{t}}^{l}<0$, it is trivial so we assume that $\underline{\mathbf{t}}^{l} \geq 0$ and that $\underline{\mathbf{x}}^{l} \geq \underline{y}_{0}$, then for $t<\underline{T}_{l}$ we have the following bound :

$$
\underline{\mathbf{t}}^{l}<\alpha \underline{y}_{0}\left(\sum_{j=0}^{l-1} \beta^{j}-\beta^{l} \sum_{j=1}^{l} \beta^{-j}\right)<0,
$$

which is a contradiction.

Now let fix $k$ such that $l \leq k \leq M$ and a time $t \in\left[\underline{T}_{k}, \min \left(T, \underline{T}_{k+1}\right)\right.$ [. Once again let us assume that $\underline{\mathbf{t}}^{l}>0$, so $g\left(\underline{\mathbf{t}}^{l}, \underline{\mathbf{x}}^{l}\right)$ is zero if $\underline{\mathbf{x}}^{l}<\underline{y}_{0}$, from the support property of $g$. In other words $g\left(\underline{\mathbf{t}}^{l}, \underline{\mathbf{x}}^{l}\right)$ is zero for $x<\beta^{l} y_{0}$. But using the fact that $\beta \leq 1$ and that $l \leq k$ it follows, in particular, that $g\left(\underline{\mathbf{t}}^{l}, \underline{\mathbf{x}}^{l}\right)$ is zero for $x<\beta^{k} y_{0}$.

With Propositions 31 and 32 in hand we now turn to the study of equation (85). At the end of the previous paragraph we explained why (85) could be rewritten as:

$$
\left[(I-\mathbb{T})\left(\mathscr{T} \mu_{0}\right)\right]\left(t, x_{2}\right)=\mu_{1}(\underline{\mathbf{t}}, \underline{\mathbf{x}}),
$$

where we recall that $\mathbb{T}$ is an operator reading under the form (128).

The idea of the resolution is to remark that the operator $\mathbb{T}$ is expressed in the variables $(\underline{\mathbf{t}}, \underline{\mathbf{x}})$ and thus the composition by $\mathbb{T}$ "costs" in terms of time of travel the time needed to make one complete circuit around the loop. Thus as we arrange the things in such a way that we can only make $M$ turns around the loop, one can always invert $I-\mathbb{T}$ by taking the Neumann serie expansion. Indeed, the terms associated with $\mathbb{T}^{j}$ with $j$ large enough "cost too much time" to appear and consequently are zero. Then we show that we can repeat exactly the same reasoning to show that we can express $\mu_{0}$ in terms of $\mu_{2}$ (the scalar component of the unknown trace $u_{2, n_{1}}$ ) and so on to express $\mu_{0}$ in terms of some $\mu_{k}$ (the scalar component of the unknown trace $u_{k, n_{1}}$ ) for $k$ arbitrarily large and in terms of the source term $g$. However, the trace $\mu_{0}$ in fact does not depend on $\mu_{k}$ for $k$ arbitrarily large, because this should say that we have made an arbitrary number of complete circuits around the loop. We thus determine $\mu_{0}$ in terms of $g$ only.

Formally, we can always invert the operator $(I-\mathbb{T})$ appearing in equation (131) by taking its Neumann serie expansion. This gives the following formal value of $\mathscr{T} \mu_{0}$ :

$$
\left(\mathscr{T} \mu_{0}\right)\left(t, x_{2}\right)=\sum_{j \geq 0} \mathbb{T}^{j} \mu_{1}(\underline{\mathbf{t}}, \underline{\mathbf{x}}) .
$$

Let us remark that the second sum in the right hand side of (132) reads :

$$
\sum_{j \geq 1}\left(F_{j}\left(\mu_{1}\right)\right)\left(\underline{\mathbf{t}}^{j}, \underline{\mathbf{x}}^{j}\right)
$$

where the operators $F_{j}\left(\mu_{1}\right)$ are some explicitly computable operators and where $\underline{\mathbf{t}}^{j}$ and $\underline{\mathbf{x}}^{j}$ denote that we made the change of variables $(t, x) \rightarrow\left(\underline{\mathbf{t}}\left(t, x_{2}\right), \underline{\mathbf{x}}\left(x_{2}\right)\right) j$ times that is:

$$
\underline{\mathbf{t}}^{j}=\underline{\mathbf{t}}^{j}\left(t, x_{2}\right):=t-\alpha \sum_{l=0}^{j} \beta^{-j} x \text { and } \underline{\mathbf{x}}^{j}=\underline{\mathbf{x}}^{j}(x):=\beta^{-j} x_{2} .
$$


Now remember that by definition of the profiles spaces (see Definition 27) we are looking for $\mu_{1}$ to be in $\mathscr{P}_{b, \leq}^{M-1}$, so using the fact that the operators $F_{j}$ are sums of derivatives (up to some multiplication by known functions), it follows that the $F_{j}\left(\mu_{1}\right)$ are in $\mathscr{P}_{b, \leq}^{M-1}$. Proposition 31 shows that $F_{j}\left(\mu_{1}\right)\left(\underline{\mathbf{t}}^{j}, \underline{\mathbf{x}}^{j}\right)$ is in $\mu_{1} \in \mathscr{P}_{b, \leq}^{M-1+j}$.

As a consequence, equation (132) in fact reads:

$$
\left(\mathscr{T} \mu_{0}\right)(t, x)=\mu_{1}(\underline{\mathbf{t}}, \underline{\mathbf{x}}),
$$

which gives a rigorous, because the Neumann expansion for $(I-\mathbb{T})$ is finite, value of $\left(\mathscr{T} \mu_{0}\right)$ in terms of the (still) unknown function $\mu_{1} \in \mathscr{P}_{b, \leq}^{M-1}$. In terms of wave packets propagation (134) tells us that $\mu_{0}$, compared to the others terms in the WKB expansion, only depends on $\mu_{1}$ which has made a complete circuit around the loop. This fact agrees whith the intuition given in paragraph 4 that the amplitude $u_{0, n_{1}}$ does not depend on the amplitudes $u_{n, n_{1}}$ for $n \geq 2$. Indeed these amplitudes do not have achieved enough complete circuits around the loop to suffer enough amplifications and consequently they remain of higher order in terms of $\varepsilon$. Let us also stress that the term depending on $\widetilde{g}$ in the right hand side of (134) starts to be evaluated in $(t, x)$ while the term depending on $\mu_{1}$ is evaluated in $(\underline{\mathbf{t}}, \underline{\mathbf{x}})$. This will be a crucial point in the following.

If we assume that $\mu_{1}$ is a known function in $\mathscr{P}_{b, \leq}^{M-1}$, then is it easy to compute $\mu_{0}$ : indeed by definition of the transport operator $\mathscr{T}, \mu_{0}$ satisfies the transport equation:

$$
\begin{cases}(\mathscr{T} \mu)\left(t, x_{2}\right)=\left(\partial_{t} \mu_{0}-\frac{\underline{\tau}}{\bar{\xi}_{2}} \partial_{2} \mu_{0}\right)\left(t, x_{2}\right)=\mu_{1}(\underline{\mathbf{t}}, \underline{\mathbf{x}}), & \text { for } \left.\left.\left(t, x_{2}\right) \in\right]-\infty, T\right] \times \mathbb{R}_{+}, \\ \mu_{0_{\mid x_{2}=0}}=0, & \text { on } t \in]-\infty, T], \\ \mu_{0_{\mid t \leq 0}}=0, & \text { on } x_{2} \in \mathbb{R}_{+},\end{cases}
$$

which can be integrated along the characteristics to obtain the value of $\mu_{0}$. Let us denote by $\mathscr{K}$ the application that to a given source term in the interior for the transport equation (135) associates the solution of the transport equation (135). We can thus write:

$$
\mu_{0}\left(t, x_{2}\right)=\mathscr{K}\left(\mu_{1}(\underline{\mathbf{t}}, \underline{\mathbf{x}})\right) .
$$

We recall that we made the hypothesis that $\frac{\underline{\underline{\xi_{2}}}}{\overline{\mathrm{N}}_{2}}<0$ (see Hypothesis 4.3), the transport operator $\mathscr{T}$ "pushes" the information away from the set $\left\{x_{2}=0\right\}$. As a consequence, integrating (135) along the characteristics show that applying the operator $\mathscr{K}$ does not destroy the property to be zero on the strip $\left.\{t \in]-\infty, T], 0<x_{2}<Y\right\}$, for some $Y>0$. This remark justify the following proposition:

Proposition 33. Let $\mathscr{K}$ be the inverse of the transport operator $\mathscr{T}$ defined previously. Then the profile spaces are invariant sets under the action of $\mathscr{K}$. More precisely if $\mu \in \mathscr{P}_{b, \leq}^{k}$, for some $k \in \mathbb{N}$, then $(\mathscr{K} \mu) \in \mathscr{P}_{b, \leq}^{k}$.

Consequently if $\mu_{1} \in \mathscr{P}_{b, \leq}^{M-1}$ then equation (136) gives, as expected, a trace $\mu_{0}$ in $\mathscr{P}_{b, \leq}^{M}$.

However (136) is not sufficient to determine $\mu_{0}$ because the trace $\mu_{1}$ is not a known function. That is why in the following paragraph, we will study the unknown trace $\mu_{1}$. We will show that it can be expressed in terms of the unknown trace $\mu_{2}$ and $\mu_{0}$.

But before that let us give some comments about the resolution of equation (85) in the case $\beta>1$.

The case $\beta>1$, the information does not approach the corner.. As for the case $\beta \leq 1$ we start by a study of the influence of the change of variables $(\underline{\mathbf{t}}, \underline{\mathbf{x}})$ on the profile sets $\mathscr{P}_{b,>}^{M-n}$. The following proposition is equivalent to Proposition 31 in the framework $\beta \leq 1$.

Proposition 34. If $\mu \in \mathscr{P}_{b,>}^{M-n}$, then $\mu(\underline{\mathbf{t}}, \underline{\mathbf{x}}) \in \mathscr{P}_{b,>}^{M-n+1}$. In particular, $\mu\left(\underline{\mathbf{t}}^{j}, \underline{\mathbf{x}}^{j}\right)$ is zero for $l \geq n+1$.

The proof of this proposition is based on the same ideas than the proof of Proposition 31 but is simpler. That is why we will not give a proof here.

Let us also note that thanks to the conditions imposed on the source term $g$, Proposition 32 is trivial in the framework $\beta>1$.

With Proposition 34 in hand it is then easy to show that the Neumann serie expansion associated with equation (131) contains a finite number of non-zero terms. One can thus reiterate the arguments described 
in the framework $\beta \leq 1$, to show that (136) also holds for $\beta>1$. Indeed in the framework $\beta \leq 1$ to establish (136) we only use Proposition 31 and 32 but we do not use explicitly the fact that $\beta \leq 1$. Also remark that Proposition 33 only uses the fact that $\frac{\tau}{\overline{\xi_{2}}}<0$ so this Proposition still holds for $\beta>1$.

7.1.5. The equation on $\mu_{n}, n>0$. In this paragraph we give an equation determining the unknown scalar part of the trace $u_{n, n_{1 \mid x_{1}=0}}$ for all $n>0$. As the reader may notice, this equation looks like equation (85) determining the unknown trace of order zero $\mu_{0}$. However for $n>0$, the equation involves some extra terms. The appearance of these terms is due to the fact that, as already noticed in paragraph 7.1.1, the amplitudes $u_{n, n_{1}}$ are not polarized for $n>0$.

As it as been done at the beginning of Paragraph 7.1 for $\mu_{0}$, to obtain this new equation we study the boundary condition (55) written for $j=n_{1}$ :

$$
B_{1}\left[\sum_{j \in \Phi\left(n_{1}\right)} u_{n, j}\right]_{\mid x_{1}=0}=\delta_{n, M+1} g
$$

And we decompose the amplitudes $\in \Phi\left(n_{1}\right) \backslash\left\{n_{4}\right\}$ and isolate the only outgoing-incoming phase $n_{4}$ to obtain:

$$
B_{1}\left[\sum_{j \in \Phi\left(n_{1}\right) \backslash\left\{n_{4}\right\}} P_{1}^{j} u_{n, j}\right]_{\mid x_{1}=0}+B_{1}\left[\sum_{j \in \Phi\left(n_{1}\right) \backslash\left\{n_{4}\right\}}\left(I-P_{1}^{j}\right) u_{n, j}\right]_{\mid x_{1}=0}=\delta_{n, M+1} g-B_{1} u_{n, n_{4} \mid x_{1}=0} .
$$

According to Proposition 17, the first term in the left hand side of (138) is in $B_{1} E_{1}^{s}\left(i \underline{\tau}, \bar{\xi}_{2}\right)$. So take the inner product of (138) by the vector $b$ introduced in Definition 12 makes this term vanish. This gives:

$$
b \cdot B_{1}\left[\sum_{j \in \Phi\left(n_{1}\right) \backslash\left\{n_{4}\right\}}\left(I-P_{1}^{j}\right) u_{n, j}\right]_{\mid x_{1}=0}=\delta_{n, M+1} b \cdot g-b \cdot B_{1} u_{n, n_{4} \mid x_{1}=0}
$$

But let us recall that from the cascade of equations (52) we know that the unpolarized part of the amplitudes of order $n$ are given by the polarized parts of the amplitudes of order $n-1$. More precisely for all $j \in \mathscr{I}_{\text {os }}$ we have:

$$
\left(I-P_{1}^{j}\right) u_{n, j}=i R_{1}^{j} L(\partial) u_{n-1, j}=i R_{1}^{j} L(\partial)\left(P_{1}^{j} u_{n-1, j}+\left(I-P_{1}^{j}\right) u_{n-1, j}\right),
$$

and using this relation in equation (139) leads us to:

$$
i b \cdot B_{1}\left[\sum_{j \in \Phi\left(n_{1}\right) \backslash\left\{n_{4}\right\}} R_{1}^{j} L(\partial) P_{1}^{j} u_{n-1, j}\right]_{\mid x_{1}=0}=b \cdot \delta_{n, M+1} g-b \cdot B_{1} u_{n, n_{4} \mid x_{1}=0}
$$

$$
-i b \cdot B_{1}\left[\sum_{j \in \Phi\left(n_{1}\right) \backslash\left\{n_{4}\right\}} R_{1}^{j} L(\partial)\left(I-P_{1}^{j}\right) u_{n-1, j}\right]_{\mid x_{1}=0} .
$$

We thus apply Proposition 29 to rewrite the left hand side in terms of the transport operator along the boundary:

$$
\mathscr{T} \mu_{n-1}=\delta_{n, M+1} b \cdot g-b \cdot B_{1} u_{n, n_{4} \mid x_{1}=0}-i b \cdot B_{1}\left[\sum_{j \in \Phi\left(n_{1}\right) \backslash\left\{n_{4}\right\}} R_{1}^{j} L(\partial)\left(I-P_{1}^{j}\right) u_{n-1, j}\right]_{\mid x_{1}=0},
$$

which tells us that the scalar unknown part of the amplitude $u_{n-1, n_{1}}$, namely $\mu_{n-1}$, satisfies the same transport equation as $\mu_{0}$ up to the extra term $-i b \cdot B_{1}\left[\sum_{j \in \Phi\left(n_{1}\right) \backslash\left\{n_{4}\right\}} R_{1}^{j} L(\partial)\left(I-P_{1}^{j}\right) u_{n-1, j}\right]_{\mid x_{1}=0}$.

However, we can reiterate the computations made in Paragraphs 7.1.1 and 7.1.3 to make the terms $b \cdot B_{1} u_{n, n_{4 \mid x_{1}=0}}$ explicit in terms of $\mu_{n-1}$ and $\mu_{n}$. This shows that $b \cdot B_{1} u_{n, n_{4} \mid x_{1}=0}$ involves $\mu_{n}$ evaluated in 
$(\underline{\mathbf{t}}, \underline{\mathbf{x}})$ and also $\mathbb{T}\left(\mathscr{T} \mu_{n-1}\right)$. We thus have:

$$
\begin{aligned}
{\left[(I-\mathbb{T})\left(\mathscr{T} \mu_{n-1}\right)\right]\left(t, x_{2}\right) } & =\delta_{n, M+1} b \cdot g\left(t, x_{2}\right)+\mu_{n}(\underline{\mathbf{t}}, \underline{\mathbf{x}}) \\
& -i b \cdot B_{1}\left[\sum_{j \in \Phi\left(n_{1}\right) \backslash\left\{n_{4}\right\}} R_{1}^{j} L(\partial)\left(I-P_{1}^{j}\right) u_{n-1, j}\right]_{\mid x_{1}=0}\left(t, x_{2}\right),
\end{aligned}
$$

and we recover (85) up to the extra term $-i b \cdot B_{1}\left[\sum_{j \in \Phi\left(n_{1}\right) \backslash\left\{n_{4}\right\}} R_{1}^{j} L(\partial)\left(I-P_{1}^{j}\right) u_{n-1, j}\right]_{\mid x_{1}=0}$ which involves the $u_{n-1, j}$ for $j \in \Phi\left(n_{1}\right) \backslash\left\{n_{4}\right\}$ (and consequently $\mu_{n-1}$ ) that is why we have to make this term more explicit in terms of $\mu_{n-1}$.

To do this we reiterate the computations of paragraph 7.1.1 to treat the last term in the right hand side of (142). First we remark that the terms $\left(I-P_{1}^{j}\right) u_{n-1, j}$ for $j \neq n_{1}, n_{4}$ are given by equation (109) when $n=2$. However the result is totally similar when $n>2$. So after having applied the operator $R_{1}^{j} L(\partial)$ and taking the trace on $\left\{x_{1}=0\right\}$ one can show that the sum on $j \in \Phi^{*}\left(n_{1}\right) \backslash n_{4}$ appearing in the right hand side of (142) depends on $\mu_{n-2}(\underline{\mathbf{t}}, \underline{\mathbf{x}}$ ) (and possibly of the source term $g$ ). That is why we write:

$$
\left[\sum_{j \in \Phi^{*}\left(n_{1}\right) \backslash\left\{n_{4}\right\}} R_{1}^{j} L(\partial)\left(I-P_{1}^{j}\right) u_{n-1, j}\right]_{\mid x_{1}=0}=\left(\Lambda_{1} \mu_{n-2}\right)(\underline{\mathbf{t}}, \underline{\mathbf{x}}),
$$

where $\Lambda_{1}$ is some explicitly computable operator which is a sum of derivatives and multiplications by scalar functions. The exact expression of $\Lambda_{1}$ is not relevant for our discussion.

The same argument holds for $\left(I-P_{1}^{n_{1}}\right) u_{n-1, n_{1}}$. It is given by (89) in the case $n=2$, but the computations are totally analogous when $n>2$. We thus can write:

$$
\left[R_{1}^{n_{1}} L(\partial)\left(I-P_{1}^{n_{1}}\right) u_{n-1, n_{1}}\right]_{\mid x_{1}=0}=\left(\Lambda_{0} \mu_{n-2}\right)\left(t, x_{2}\right),
$$

where $\Lambda_{0}$ is an operator which acts like $\Lambda_{1}$.

So (142) in its final form reads:

$$
\left[(I-\mathbb{T}) \mathscr{T} \mu_{n-1}\right]\left(t, x_{2}\right)=\delta_{n, M+1} \widetilde{g}\left(t, x_{2}\right)+\mu_{n}(\underline{\mathbf{t}}, \underline{\mathbf{x}})+\left(\Lambda \mu_{n-2}\right)\left(t, x_{2}\right),
$$

where we set $\widetilde{g}:=b \cdot g$ and $\Lambda u:=\left(\Lambda_{0} u\right)(t, x)+\left(\Lambda_{1} u\right)(\underline{\mathbf{t}}, \underline{\mathbf{x}})$. The only point to keep in mind about $\Lambda$ is that the profile sets $\mathscr{P}_{b, \leq}^{k}$ and $\mathscr{P}_{b,>}^{k}$ are invariant sets for the operator $\Lambda$ because this operator is a sum (up to some multiplications) of derivatives and thus does not modify supports properties.

7.1.6. End of the resolution of equation (85). In this paragraph we describe the end of the resolution of equation (85) when $\beta \leq 1$. As we will see this resolution does not take into account the precise value of $\beta$ but only needs Proposition 31. Proposition 31 has its equivalent when $\beta>1$ (that is to say Proposition 34). So the proof given here will extend to the case $\beta>1$.

To save some notations, as $\beta$ is not relevant in this discussion, we will denote $\mathscr{P}_{b}^{M-n}$ in place of $\mathscr{P}_{b, \leq}^{M-n}$.

The end of paragraph 7.1.4 shows that (85) in fact reads:

$$
\mu_{0}=\mathscr{K}\left(\mu_{1}(\underline{\mathbf{t}}, \underline{\mathbf{x}})\right),
$$

from which we deduced that if $\mu_{1} \in \mathscr{P}_{b}^{M-1}$ then $\mu_{0} \in \mathscr{P}_{b}^{M}$. Because of the definition of the profile spaces (see Definition 27) we assume that for all $1 \leq n \leq M+1, \mu_{n} \in \mathscr{P}_{b}^{M-n}$. Our aim is here to show that equation (144) defines a unique $\mu_{0} \in \mathscr{P}_{b}^{M}$ and to express this solution $\mu_{0}$ as a function of the source term $g$.

Using the analysis described in the previous paragraph we are now able to give more informations about the unknown $\mu_{1}$. Indeed, (143) written for $n=2$ reads:

$$
\left[(I-\mathbb{T}) \mathscr{T} \mu_{1}\right]\left(t, x_{2}\right)=\delta_{M, 1} \widetilde{g}\left(t, x_{2}\right)+\mu_{2}(\underline{\mathbf{t}}, \underline{\mathbf{x}})+\left(\Lambda \mu_{0}\right)\left(t, x_{2}\right) .
$$


Using the fact that $\mu_{0} \in \mathscr{P}_{b}^{M}$ and the fact that $\Lambda$ keeps $\mathscr{P}_{b}^{M}$ invariant we obtain that the term $\left(\Lambda \mu_{0}\right)(t, x)$ appearing in (145) is in $\mathscr{P}_{b}^{M}$. As in paragraph 7.1.4, we write the Neumann serie expansion associated to (145):

$$
\left(\mathscr{T} \mu_{1}\right)\left(t, x_{2}\right):=\delta_{M, 1} \sum_{j \geq 0} \mathbb{T}^{j}\left(\widetilde{g}\left(t, x_{2}\right)\right)+\sum_{j \geq 0} \mathbb{T}^{j}\left(\mu_{2}(\underline{\mathbf{t}}, \underline{\mathbf{x}})\right)+\sum_{j \geq 0} \mathbb{T}^{j}\left(\Lambda \mu_{0}\left(t, x_{2}\right)\right) .
$$

Recall that each iteration of $\mathbb{T}$ induces the evaluation in $(\underline{\mathbf{t}}, \underline{\mathbf{x}})$. So from Proposition $31, \mathbb{T}^{j}\left(\mu_{2}(\underline{\mathbf{t}}, \underline{\mathbf{x}})\right)$ is zero for all $j \geq 2$ and $\mathbb{T}^{j}\left(\Lambda \mu_{0}\left(t, x_{2}\right)\right)$ as soon as $j \geq 1$ (beacause $\mu_{2} \in \mathscr{P}_{b}^{M-2}$ and $\Lambda \mu_{0} \in \mathscr{P}_{b}^{M}$ ). From Proposition $32, \mathbb{T}^{j}\left(\widetilde{g}\left(t, x_{2}\right)\right)$ is zero for all $j \geq 2$. So the Neumann serie expansion only contains a finite number of terms and reads:

$$
\left(\mathscr{T} \mu_{1}\right)\left(t, x_{2}\right):=\delta_{M, 1}\left(\widetilde{g}\left(t, x_{2}\right)+\mathbb{T} \tilde{g}(\underline{\mathbf{t}}, \underline{\mathbf{x}})\right)+\mu_{2}(\underline{\mathbf{t}}, \underline{\mathbf{x}})+\mathbb{T} \mu_{2}\left(\underline{\mathbf{t}}^{2}, \underline{\mathbf{x}}^{2}\right)+\left(\Lambda \mu_{0}\right)\left(t, x_{2}\right) .
$$

Up to the source term in the interior, $\mu_{0}$ and $\mu_{1}$ solve the same transport equation so we can write:

$$
\mu_{1}\left(t, x_{2}\right)=\mathscr{K}\left(\delta_{M, 1}\left(\widetilde{g}\left(t, x_{2}\right)+\mathbb{T} \widetilde{g}(\underline{\mathbf{t}}, \underline{\mathbf{x}})\right)+\mu_{2}(\underline{\mathbf{t}}, \underline{\mathbf{x}})+\mathbb{T} \mu_{2}\left(\underline{\mathbf{t}}^{2}, \underline{\mathbf{x}}^{2}\right)+\Lambda \mu_{0}\left(t, x_{2}\right)\right) .
$$

where we recall that $\mathscr{K}$ is the operator that to a source term $f$ associates the solution of the transport equation $\mathscr{T} u=f$. When we evaluate (147) in $(\underline{\mathbf{t}}, \underline{\mathbf{x}})$ we obtain, using Propositions 31 and 32, that:

$$
\mu_{1}(\underline{\mathbf{t}}, \underline{\mathbf{x}})=\mathscr{K}\left(\delta_{M, 1} \widetilde{g}(\underline{\mathbf{t}}, \underline{\mathbf{x}})+\mu_{2}\left(\underline{\mathbf{t}}^{2}, \underline{\mathbf{x}}^{2}\right)\right) .
$$

Let us stress that (148) written in this form is not true. Indeed in this formulation, we used the fact that $\mathscr{K}$ and the evaluation $(\underline{\mathbf{t}}, \underline{\mathbf{x}})$ commute which is is clearly false. However we are in this purpose only interested in the profile spaces in which the terms in the right hand side of (148) lie and we are not really interested in their precise values. As from Proposition 33, $\mathscr{K}$ keeps the spaces $\mathscr{P}_{b}^{M-n}$ invariant, our abuse of notations is not so important (as far as the spaces $\mathscr{P}_{b}^{M-n}$ are concerned). But the reader has to keep in mind that if he really wants to compute the WKB expansion it is necessary to apply $\mathscr{K}$ and then make the evaluation $(\underline{\mathbf{t}}, \underline{\mathbf{x}})$.

From equations (144) and (148) we deduce that

$$
\mu_{0}(t, x)=\mathscr{K} \mu_{1}(\underline{\mathbf{t}}, \underline{\mathbf{x}})=\mathscr{K}^{2}\left(\delta_{M, 1} \widetilde{g}(\underline{\mathbf{t}}, \underline{\mathbf{x}})+\mu_{2}\left(\underline{\mathbf{t}}^{2}, \underline{\mathbf{x}}^{2}\right)\right) .
$$

As a consequence $\mu_{0}$ can be expressed in terms of $\mu_{2}$ (and $\mu_{2}$ only) evaluated in a time corresponding to two complete circuits around the loop. Once again this observation meets the intuition given in Section 4.

More generally, we can repeat exactly the same arguments for all $n \geq 1$ to obtain that: for all $n>0$ :

$$
\mu_{n}\left(t, x_{2}\right)=\mathscr{K}\left(\delta_{n, M} \sum_{j=0}^{M} \mathbb{T}^{j} \widetilde{g}\left(\underline{\mathbf{t}}^{j}, \underline{\mathbf{x}}^{j}\right)+\sum_{j=1}^{\min (n+1, M)} \mathbb{T}^{j-1} \mu_{n+1}\left(\underline{\mathbf{t}}^{j}, \underline{\mathbf{x}}^{j}\right)+\sum_{j=0}^{\min (n-1, M)} \mathbb{T}^{j} \Lambda \mu_{n-1}\left(\underline{\mathbf{t}}^{j}, \underline{\mathbf{x}}^{j}\right)\right)
$$

If for all $0<n \leq M+1, \mu_{n} \in \mathscr{P}_{b}^{M-n}$, these formulas make sense for $0<n \leq M$ (because we can ensure that the Neumann serie expansion contains a finite number of terms). We deduce from (150) that for all $l \leq M$ :

$$
\mu_{n}\left(\underline{\mathbf{t}}^{l}, \underline{\mathbf{x}}^{l}\right)=\mathscr{K}\left(\delta_{n, M} \sum_{j=l}^{M} \mathbb{T}^{j} \widetilde{g}\left(\underline{\mathbf{t}}^{j}, \underline{\mathbf{x}}^{j}\right)+\sum_{j=l+1}^{\min (n+1, M)} \mathbb{T}^{j-1} \mu_{n+1}\left(\underline{\mathbf{t}}^{j}, \underline{\mathbf{x}}^{j}\right)+\sum_{j=l}^{\min (n-1, M)} \mathbb{T}^{j} \Lambda \mu_{n-1}\left(\underline{\mathbf{t}}^{j}, \underline{\mathbf{x}}^{j}\right)\right)
$$

from which it follows that for all $n<M$, each $\mu_{n}\left(\underline{\mathbf{t}}^{n}, \underline{\mathbf{x}}^{n}\right)$ is equal to $\mu_{n+1}\left(\underline{\mathbf{t}}^{n+1}, \underline{\mathbf{x}}^{n+1}\right)$, and that for $n=M$, $\mu_{n}\left(\underline{\mathbf{t}}^{n}, \underline{\mathbf{x}}^{n}\right)$ is equal to $g\left(\underline{\mathbf{t}}^{M}, \underline{\mathbf{x}}^{M}\right)$. A simple iteration in (149) shows that:

$$
\mu_{0}\left(t, x_{2}\right)=\mathscr{K}^{M} \widetilde{g}\left(\underline{\mathbf{t}}^{M}, \underline{\mathbf{x}}^{M}\right),
$$

equation determining in a unique way $\mu_{0} \in \mathscr{P}_{b}^{M}$ in terms of the known source term $g$. This concludes the resolution of equation (85). 
with homogeneous initial condition. As $\ell_{1} \in \mathscr{I}_{\text {oi }}$ this transport equation needs a boundary condition on $\partial \Omega_{2}$ to be solved. This boundary condition is given by cascade (55) written for $n=0$ and $k=n_{1}$ :

$$
B_{2}\left[\sum_{j \in\left(\Psi\left(n_{1}\right) \cap \mathscr{I}_{o s}\right) \backslash\left\{n_{1}\right\}} u_{0, j}+U_{0, n_{1}, 2_{\mid X_{2}=0}}\right]_{\mid x_{2}=0}=-B_{2} u_{0, n_{1 \mid x_{2}=0}}, \quad \text { if } n_{1} \in \mathfrak{R}_{2},
$$

or

$$
B_{2}\left[\sum_{j \in\left(\Psi\left(n_{1}\right) \cap \mathscr{I}_{o s}\right) \backslash\left\{n_{1}\right\}} u_{0, j}\right]_{\mid x_{2}=0}=-B_{2} u_{0, n_{1 \mid x_{2}=0}}, \quad \text { if } n_{1} \in \mathscr{R}_{2} \backslash \mathfrak{R}_{2} .
$$

We reiterate the computations made for $u_{0, n_{2}}$ (see the beginning of Section 7 ). We use the uniform KreissLopatinskii condition and then we multiply by $P_{2}^{\ell_{1}}$ to obtain in both cases:

$$
u_{0, \ell_{1 \mid x_{2}=0}}=-P_{2}^{\ell_{1}} \phi_{2}^{n_{1}} B_{2} u_{0, n_{1 \mid x_{2}=0}} .
$$

Let us point that (152) meets with the intuition described in Section 4 that $\mu_{0}$ depends on the initial

Now that the value of the trace $\mu_{0}$ is determine it end up the determination of the zero order amplitudes of the loop indices. Indeed these four amplitudes are given by (66)-(68)-(70) and (71). The initialization of the resolution of the cascades (52)-(55) and (56) is finished and we now have to determine all the others zero

7.2. Determination of the others amplitudes in the WKB expansion. Now that at least one mplitude is determined to determine all the others it is sufficient to find an order of resolution. The main

The construction and, in particular, the partial order of resolution used are exactly the same as in [4] so

7.2.1. Determination of the oscillating amplitudes. Thanks to Proposition 23 the set of indices $\mathscr{I}_{\text {os }} \backslash\left\{n_{j}\right\}$ can be expressed as a partition (see (45) and 3 for more details). We will here describe the us say $A_{a_{1}}$. The determination of the amplitudes in the others sets composing (45) is similar and will not be

Let us denote by $\ell_{i}:=\left(\ell_{1}, \ell_{2}, \ldots, \ell_{p}\right)$ the type $V$ sequence linking $n_{1}$ to $\underline{i}$ (see Definition 18 and Proposition 23). By definition of the tree $A_{a_{1}}$ (see again Definition 18), $\ell_{1}=a_{1}$ and as a consequence $\ell_{1}$ is associated to an outgoing-incoming group velocity, $\ell_{2}$ to an incoming-outgoing group velocity and so one (note that $\ell_{p}$ is

Before to determine the amplitude associated to $\underline{i}$ we will have to determine all the amplitudes in the sequence $\ell_{i}$. As it has been done to the loop's amplitudes (see Paragraph 7.1), thanks to the fact that $u_{0, \ell_{1}}$

So we have to solve the transport equation:

$$
\begin{cases}\left(\partial_{t}+v_{\ell_{1}} \cdot \nabla_{x}\right) u_{0, \ell_{1}}=0, & \text { in } \Omega_{T} \\ u_{0, \ell_{1 \mid x_{2}=0}}=-P_{2}^{\ell_{1}} \phi_{2}^{n_{1}} B_{2} u_{0, n_{1 \mid x_{2}=0}}, & \text { on } \partial \Omega_{2, T} \\ u_{0, \ell_{1 \leq 0}}=0, & \text { on } \Omega\end{cases}
$$

this equation can be integrate along the characteristics (because $u_{0, n_{1}}$ and its trace on $\partial \Omega_{2, T}$ ) are known) to determine $u_{0, \ell_{1}}$. It is interesting to remark that if $t<\underline{T}_{M}$ then $u_{0, n_{1}}$ is zero and so do $u_{0, \ell_{1}}$. As a consequence the amplification of order $M+1$ only spreads to the amplitudes away from the loop for $t \geq \underline{T}_{M}$ (that is after 
$M$ complete circuits). Also note that as $u_{0, n_{1 \mid x_{2}=0}}$ vanishes in a neighborhood of $x_{1}=0$ so do $u_{0, \ell_{1 \mid x_{2}=0}}$ and by resolution of of the transport equation $u_{0, \ell_{1 \mid x_{1}=0}}$ is zero near $x_{2}=0$.

Then we construct $u_{0, \ell_{2}}$. In view of the definition of the tree $A_{\ell_{1}}, \ell_{2} \in \mathscr{I}_{\text {io }}$ so $u_{0, \ell_{2}}$ is solution of:

$$
\begin{cases}\left(\partial_{t}+v_{\ell_{2}} \cdot \nabla_{x}\right) u_{0, \ell_{2}}=0, & \text { in } \Omega_{T}, \\ u_{0, \ell_{\mid x_{1}=0}}=-P_{1}^{\ell_{2}} \phi_{1}^{\ell_{1}} B_{1} u_{0, \ell_{1 \mid x_{1}}=0}, & \text { on } \partial \Omega_{1, T}, \\ u_{0, \ell_{\mid t \leq 0}}=0, & \text { on } \Omega .\end{cases}
$$

This equation uniquely determines $u_{0, \ell_{2}}$ because $u_{0, \ell_{1 \mid x}=0}$ is known and we have $u_{0, \ell_{1 \mid x_{2}=0}}=0$ for $x_{1}$ small enough. We then proceed recursively to determine all the $u_{0, \ell_{k}}, 3 \leq k \leq p$.

We now turn to the construction of $u_{0, \underline{i}}$. We have to distinguish two cases according to the parity of $p$. Let us assume that $p$ is even. Then by definition of $A_{\ell_{1}}$ we have $\underline{i} \in \mathscr{I}_{o i} \cup \mathscr{I}_{i i}$. If $\underline{i} \in \mathscr{I}_{o i}$ the amplitude $u_{0, \underline{i}}$ solves:

$$
\begin{cases}\left(\partial_{t}+v_{\underline{i}} \cdot \nabla_{x}\right) u_{0, \underline{i}}=0, & \text { in } \Omega_{T}, \\ u_{0, \underline{i}_{\mid x_{2}=0}}=-P_{2}^{\ell_{2}} \phi_{2}^{\ell_{p}} B_{2} u_{0, \ell_{p \mid x_{2}=0}}, & \text { on } \partial \Omega_{2, T}, \\ u_{0, \underline{i}_{\mid t \leq 0}}=0, & \text { on } \Omega,\end{cases}
$$

if $\underline{i} \in \mathscr{I}_{o i}$ and we proceed as for $u_{0, \ell_{1}}$.

If $\underline{i} \in \mathscr{I}_{i i}$, then $u_{0, i}$ solves the same transport equation but this time it needs boundary conditions on both sides of the boundary. The boundary condition on $\partial \Omega_{2}$ is unchanged. Concerning the boundary condition on $\partial \Omega_{1}$ it reads (recall that incoming-incoming phases do not create new phases):

$$
B_{1} u_{0, \underline{i}_{\mid x_{1}=0}}=0 .
$$

As $\underline{i} \in \mathscr{I}_{i i}, u_{0, \underline{i} x_{1}=0} \in E_{1}^{s}\left(i \underline{\tau}, \xi_{2}^{\underline{i}}\right)$, where $\xi_{2}^{\underline{i}}$ is the ordinate of $f^{i}$, so thanks to the uniform Kreiss-Lopatinskii we obtain $u_{0, \underline{i}_{\mid x_{1}=0}}=0$. Consequently $u_{0, \underline{i}}$ solves :

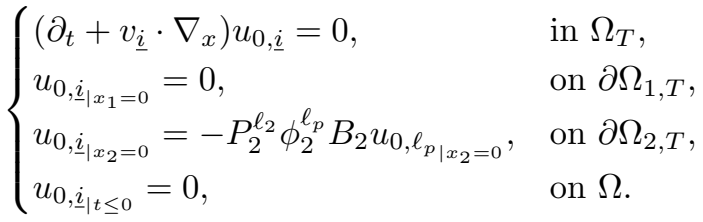

This equation can be solved with a highly regular solution (which is needed to compute the higher order amplitudes $u_{n, \underline{i}}$ as they involve derivatives of $\left.u_{0, \underline{\underline{i}}}\right)$ because $u_{0, \ell_{p \mid x_{2}=0}}$ is zero at $x_{1}=0$. If $u_{0, \ell_{p \mid x_{2}=0}}$ is not zero at $x_{1}=0$ then we only recover $u_{0, \underline{i}} \in L^{2}(\Omega)$ (see [4] for more details).

7.2.2. Determination of the evanescent amplitudes. In this paragraph we conclude the construction of the leading order of the geometric optics expansion by giving elements of proof to construct an arbitrary evanescent amplitude associated to the index $\underline{i}$ lying in the tree $A_{a_{1}}$. Without loss of generality we assume that this amplitude is evanescent for the side $\partial \Omega_{1}$. Proposition 24 implies that the type $V$ sequence $\ell_{\underline{i}}:=\left(\ell_{1}, \ell_{2}, \ldots, \ell_{p}\right)$ linking $n_{1}$ to $\underline{i}$ has an odd number of terms. Moreover, from the previous paragraph all the $\ell_{j}$ has already been constructed. We then recall the following lemma due to [12] which states that evanescent equations in the cascade (52) can be solved in the profile space $\mathbb{P}_{e v, 1}$ :

Lemma 35. For $j=1,2$, and $\underline{k} \in \mathfrak{R}_{i}$, let

$$
\begin{aligned}
& \mathbb{P}_{e v, j}^{\underline{k}} U\left(X_{j}\right):=e^{X_{j} \mathscr{A}_{j}\left(\underline{\tau}, \xi_{3-j}^{k}\right)} P_{s, j}^{\underline{k}} U(0), \\
& \mathbb{Q}_{e v, j}^{\frac{k}{e}} F\left(X_{j}\right):=\int_{0}^{X_{j}} e^{\left(X_{j}-s\right) \mathscr{A}_{j}\left(\underline{\tau}, \xi_{3-j}^{k}\right)} P_{s, j}^{\underline{k}} A_{j}^{-1} F(s) d s-\int_{X_{j}}^{+\infty} e^{\left(X_{j}-s\right) \mathscr{A}_{j}\left(\underline{\tau}, \xi_{3-j}^{k}\right)} P_{u, j}^{\underline{k}} A_{i}^{-1} F(s) d s .
\end{aligned}
$$

Then, for all $F \in \mathbb{P}_{e v, j}$ the equation:

$$
L_{\underline{k}}\left(\partial_{X_{j}}\right) U=F,
$$


admits a solution in $\mathbb{P}_{e v, i}$. Moreover, this solution reads:

$$
U=\mathbb{P} \frac{k}{e v, i} U+\mathbb{Q} \frac{k}{e v, i} F
$$

This lemma tells us that to construct any evanescent amplitude for the side $\partial \Omega_{1}$ it is in fact sufficient to know the value of the trace the solution on $\left\{X_{1}=0\right\}$. Following [12], to determine this trace we study the boundary condition (55) written for $k=\underline{i}$ and $n=0$ from which we deduce the value of the "double" trace on $\left\{X_{1}=x_{1}=0\right\}$. Indeed thanks to the uniform Kreiss-Lopatinskii condition we obtain:

$$
U_{0, \underline{i}, k_{\mid X_{1}=x_{1}=0}}=-P_{e v, 1}^{\underline{i}} \phi_{1}^{\frac{i}{1}} B_{1} u_{0, \ell_{p \mid x_{1}=0}},
$$

where we recall that the right hand side has already been determined.

Then as in [12], we are free to straighten the "double" trace into a "single" one by setting (for example):

$$
U_{0, \underline{i}, k_{\mid X_{1}=0}}=-\chi\left(x_{1}\right) S_{e v, 1}^{i} B_{1} u_{0, \ell_{p \mid x_{1}=0}},
$$

where $\chi$ is some function in $\mathscr{D}(]-1,+\infty[)$ satisfying $\chi(0)=1$.

It is interesting to remark that evanescent amplitudes suffer the same amplification as the oscillating ones and this even if the degeneracy of the uniform Kreiss-Lopatinskii condition is in the hyperbolic region and thus not related to complex phases. Such a behaviour is not observable for the hyperbolic boundary value problem in the half space because the possible evanescent modes are "turned on" by the source term and can not be "turned on" by oscillating modes. As for the oscillating amplitudes, one can show that evanescent amplitudes of order zero are zero for $t<\underline{T}_{M}$.

7.3. Construction of the higher order terms and summary. As for the construction of the leading order of the WKB expansion, we have to distinguish the case $\beta \leq 1$ and the case $\beta>1$. But once again we only need Proposition 31 (resp. Proposition 34) to conclude if $\beta \leq 1$ (resp. $\beta>1$ ) so the proof is the same in both cases. We will assume that $\beta \leq 1$ and we continue to note $\mathscr{P}_{b}^{M-n}$ for $\mathscr{P}_{b, \leq}^{M-n}$.

7.3.1. The term of order one. Once the amplitudes of order zero, and more precisely the keystone $\mu_{0}$, are determined we can repeat our method of construction to determine the amplitudes of order one. As for the leading order, we start by the determination of the indices of the loop, that is the $n_{j}$ for $j=1, \ldots, 4$. We remark that equations (89)-(106)-(107) and (108) give the unpolarized part of the $u_{1, n_{j}}, j=1, \ldots, 4$ in terms of $\mu_{0}$ (which at this step of the proof is a known function). Similarly the values of the polarized parts are determined in terms of the unknown trace $\mu_{1}$.

So it remains to determine the unknown trace $\mu_{1}$ to construct the amplitudes of order one for the loop's elements. Noticing that the unpolarized part of the amplitudes for the indices outside of the loop are determined (because from (86) they only depend on the associated amplitude of order zero) and reiterating the same computations as in paragraph 7.2 determines their polarized part in terms of $\mu_{1}$. As a consequence we just have to determine $\mu_{1}$ to construct all the oscillating amplitudes of order one.

To do this from paragraph 7.1.4, $\mu_{1}$ satisfies equation (147), that is:

$$
\left(\mathscr{T} \mu_{1}\right)\left(t, x_{2}\right):=\delta_{M, 1}\left(\widetilde{g}\left(t, x_{2}\right)+\mathbb{T} \tilde{g}(\underline{\mathbf{t}}, \underline{\mathbf{x}})+\mathbb{T}^{2} \widetilde{g}\left(\underline{\mathbf{t}}^{2}, \underline{\mathbf{x}}^{2}\right)\right)+\left(\mu_{2}(\underline{\mathbf{t}}, \underline{\mathbf{x}})+\mathbb{T} \mu_{2}\left(\underline{\mathbf{t}}^{2}, \underline{\mathbf{x}}^{2}\right)\right)+\Lambda \mu_{0}\left(t, x_{2}\right) .
$$

where $\mu_{2}$, appearing in the right hand side, is assumed to be $\mathscr{P}_{b}^{M-2}$ and where we used the notations of paragraph 7.1.4. From (162), after integration along the characteristics, we deduce that:

$$
\mu_{1}(t, x)=\mathscr{K}\left(\delta_{M, 1} \sum_{k=0}^{1} \mathbb{T}^{k} \widetilde{g}\left(\underline{\mathbf{t}}^{k}, \underline{\mathbf{x}}^{k}\right)+\Lambda \mu_{0}(t, x)\right)+\delta_{M \geq 2} \mathscr{K}\left(\sum_{k=1}^{\min (M, 2)} \mathbb{T}^{k-1} \mu_{2}\left(\underline{\mathbf{t}}^{k}, \underline{\mathbf{x}}^{k}\right)\right) .
$$

In this equation the first term in the right hand side is a known function which only depends on $\widetilde{g}$. So to express $\mu_{1}$ in terms of $\widetilde{g}$ we just have to express $\mu_{2}(\underline{\mathbf{t}}, \underline{\mathbf{x}})$ and $\mu_{2}\left(\underline{\mathbf{t}}^{2}, \underline{\mathbf{x}}^{2}\right)$ in terms of $\widetilde{g}$. In view to do so, we use equation (143) written for $n=3$ to obtain:

$$
\mu_{2}(t, x)=\mathscr{K}\left(\delta_{M, 2} \sum_{k=0}^{2} \mathbb{T}^{k} \widetilde{g}\left(\underline{\mathbf{t}}^{k}, \underline{\mathbf{x}}^{k}\right)+\sum_{k=1}^{\min (M, 3)} \mathbb{T}^{k-1} \mu_{3}\left(\underline{\mathbf{t}}^{k}, \underline{\mathbf{x}}^{k}\right)\right)+\mathscr{K}\left(\Lambda \mu_{1}(t, x)+\mathbb{T} \Lambda \mu_{1}(\underline{\mathbf{t}}, \underline{\mathbf{x}})\right)
$$


and a new difficulty appears in (164) compared to (136). Indeed in (164) two terms, while there is only one in (136) (in terms of $\mu_{0}$, depend on $\mu_{1}$ each of them lying respectively in $\mathscr{P}_{b}^{M}$ and in $\mathscr{P}_{b}^{M-1}$. So a priori they are not zero. However in (163) we are not interested in $\mu_{2}$ but we are interested in $\mu_{2}(\underline{\mathbf{t}}, \underline{\mathbf{x}})$ and $\mu_{2}\left(\underline{\mathbf{t}}^{2}, \underline{\mathbf{x}}^{2}\right)$. A simple change of variables in (164) shows that these quantities are given by:

$$
\begin{aligned}
\mu_{2}(\underline{\mathbf{t}}, \underline{\mathbf{x}}) & =\mathscr{K}\left(\delta_{M, 2} \sum_{k=1}^{2} \mathbb{T}^{k-1} \widetilde{g}\left(\underline{\mathbf{t}}^{k}, \underline{\mathbf{x}}^{k}\right)+\sum_{k=2}^{\min (M, 3)} \mathbb{T}^{k-2} \mu_{3}\left(\underline{\mathbf{t}}^{k}, \underline{\mathbf{x}}^{k}\right)\right)+\mathscr{K} \Lambda \mu_{1}(\underline{\mathbf{t}}, \underline{\mathbf{x}}), \\
\mu_{2}\left(\underline{\mathbf{t}}^{2}, \underline{\mathbf{x}}^{2}\right) & =\mathscr{K}\left(\delta_{M, 2} \widetilde{g}\left(\underline{\mathbf{t}}^{2}, \underline{\mathbf{x}}^{2}\right)+\delta_{M \geq 3} \mu_{3}\left(\underline{\mathbf{t}}^{3}, \underline{\mathbf{x}}^{3}\right)\right),
\end{aligned}
$$

where for $I \subset \mathbb{R}, \delta_{I}$ is the characteristic function of $I$. Now let us make the change of variables $(\underline{\mathbf{t}}, \underline{\mathbf{x}})$ in (163), it follows that :

$$
\mu_{1}(\underline{\mathbf{t}}, \underline{\mathbf{x}})=\delta_{M, 1} \mathscr{K}(\widetilde{g}(\underline{\mathbf{t}}, \underline{\mathbf{x}}))+\delta_{M \geq 2} \mathscr{K} \mu_{2}\left(\underline{\mathbf{t}}^{2}, \underline{\mathbf{x}}^{2}\right)
$$

that is to say :

$$
\mathscr{K} \Lambda \mu_{1}(\underline{\mathbf{t}}, \underline{\mathbf{x}})=\left\{\begin{array}{l}
\mathscr{K} \Lambda \mathscr{K} \widetilde{g}(\underline{\mathbf{t}}, \underline{\mathbf{x}}), \text { if } M=1, \\
\mathscr{K} \Lambda \mathscr{K}^{2}\left(\delta_{M, 2} \widetilde{g}\left(\underline{\mathbf{t}}^{2}, \underline{\mathbf{x}}^{2}\right)+\delta_{M \geq 3} \mu_{3}\left(\underline{\mathbf{t}}^{3}, \underline{\mathbf{x}}^{3}\right)\right), \text { if } M>1,
\end{array}\right.
$$

and we are free to reinject (167) in (165) and then to reinject (165) in (163) to obtain that :

$$
\mu_{1}(t, x)=\left\{\begin{array}{l}
\mathscr{K}\left(\widetilde{g}(t, x)+\mathbb{T} \widetilde{g}(\underline{\mathbf{t}}, \underline{\mathbf{x}})+\Lambda \mu_{0}(t, x)\right)+\mathscr{K}^{2} \Lambda \mathscr{K} \widetilde{g}(\underline{\mathbf{t}}, \underline{\mathbf{x}}), \text { if } M=1, \\
\mathscr{K} \Lambda \mu_{0}(t, x) \\
+\mathscr{K}^{2}\left(\delta_{M, 2} \sum_{k=1}^{2} \mathbb{T}^{k-1} \widetilde{g}\left(\underline{\mathbf{t}}^{k}, \underline{\mathbf{x}}^{k}\right)+\sum_{k=2}^{\min (M, 3)} \mathbb{T}^{k-2} \mu_{3}\left(\underline{\mathbf{t}}^{k}, \underline{\mathbf{x}}^{k}\right)\right) \quad \text { if } M>1 . \\
+\mathscr{K}^{2} \Lambda \mathscr{K}^{2}\left(\delta_{M, 2} \widetilde{g}\left(\underline{\mathbf{t}}^{2}, \underline{\mathbf{x}}^{2}\right)+\delta_{M \geq 3} \mu_{3}\left(\underline{\mathbf{t}}^{3}, \underline{\mathbf{x}}^{3}\right)\right) \\
+\mathscr{K} \mathbb{T} \mathscr{K}\left(\delta_{M, 2} \widetilde{g}\left(\underline{\mathbf{t}}^{2}, \underline{\mathbf{x}}^{2}\right)+\delta_{M \geq 3} \mu_{3}\left(\underline{\mathbf{t}}^{3}, \underline{\mathbf{x}}^{3}\right)\right)
\end{array}\right.
$$

Equation (168) ends the discussion in the particular case $M=1$. To treat the case $M>1$, let us remark that in (168) the unknown part of the right hand side of equation depends on $\mu_{3}\left(\underline{\mathbf{t}}^{2}, \underline{\mathbf{x}}^{2}\right)$ and possibly on $\mu_{3}\left(\underline{\mathbf{t}}^{3}, \underline{\mathbf{x}}^{3}\right)$. So we are exactly in the same situation as in equation (163) with $\mu_{3}\left(\underline{\mathbf{t}}^{2}, \underline{\mathbf{x}}^{2}\right), \mu_{3}\left(\underline{\mathbf{t}}^{3}, \underline{\mathbf{x}}^{3}\right)$ in place of $\mu_{2}(\underline{\mathbf{t}}, \underline{\mathbf{x}}), \mu_{2}\left(\underline{\mathbf{t}}^{2}, \underline{\mathbf{x}}^{2}\right)$ and we can thus repeat the same computations to express $\mu_{1}$ in terms of $\mu_{4}\left(\underline{\mathbf{t}}^{3}, \underline{\mathbf{x}}^{3}\right)$ and $\mu_{4}\left(\underline{\mathbf{t}}^{4}, \underline{\mathbf{x}}^{4}\right)$ and so on.

A tedious (but not difficult) reiterative process shows that for all $M>1, \mu_{1}$ is given by :

$$
\begin{aligned}
\mu_{1}\left(t, x_{2}\right) & =\mathscr{K} \Lambda \mu_{0}\left(t, x_{2}\right)+\mathscr{K}^{M}\left(\widetilde{g}\left(\underline{\mathbf{t}}^{M-1}, \underline{\mathbf{x}}^{M-1}\right)+\mathbb{T} \widetilde{g}\left(\underline{\mathbf{t}}^{M}, \underline{\mathbf{x}}^{M}\right)\right) \\
& +\sum_{k=0}^{M-1} \mathscr{K}^{M+1-k} \Lambda \mathscr{K}^{k+1} \widetilde{g}\left(\underline{\mathbf{t}}^{M}, \underline{\mathbf{x}}^{M}\right)+\sum_{k=1}^{M-1} \mathscr{K}^{k} \mathbb{T} \mathscr{K}^{M-k} \widetilde{g}\left(\underline{\mathbf{t}}^{M}, \underline{\mathbf{x}}^{M}\right),
\end{aligned}
$$

equation which determines in a unique way $\mu_{1}$ in terms of $\tilde{g}$ and the known operators $\Lambda$, $\mathbb{T}$. This completes the construction of $\mu_{1}$ and more generally of the amplitudes of order one in the $W K B$ expansion.

Moreover, all the terms composing (169) are in $\mathscr{P}_{b}^{M}$, except $\widetilde{g}\left(\underline{\mathbf{t}}^{M-1}, \underline{\mathbf{x}}^{M-1}\right)$ which is in $\mathscr{P}_{b}^{M-1}$. Thus $\mu_{1}$ defined by (169) is an element of $\mathscr{P}_{b}^{M-1}$, so it is in the good profile space. To conclude this discussion let us remark that before the time $\underline{T}_{M}, \mu_{1}$ (and consequently the $u_{1, n_{j}}$ ) only depends on the information that was initially present and which has made $M-1$ complete circuits around the loop.

Once the amplitudes for indices associated to the loop are known, the construction of the amplitudes which do not lie on the loop follows the same kind of arguments as those given in paragraph 7.2. A precise construction will not be given here and we refer to [4] for more details.

7.3.2. Summary : the construction of higher order terms. In this paragraph we sketch some elements about the construction of the amplitudes of higher order in view to give a summary of the previous construction. Let us assume that the amplitudes of order less that $n-1$ have already been determinated, our aim is to construct the amplitude of order $n$.

i) First of all, from the cascade of equations (52), we know that the unpolarized part of the oscillating amplitudes of order $n$ is known. So we only have to determine the polarized part of the oscillating amplitudes. 
Moreover, from Lemma 35 and the definition of the operator $\mathbb{Q}_{e v 1}$ (resp. $\mathbb{Q}_{e v 2}$ ), see (159), concerning the evanescent amplitudes, we will only have to determine $\mathbb{P}_{e v 1}\left(\right.$ resp. $\left.\mathbb{P}_{e v 2}\right)$.

ii) To determine the polarized part of the oscillating amplitudes of order $n$, we start, as it has been done in paragraphs 7.1 and 7.3.1 by the determination of the loop's indices. We thus reiterate the computations of paragraph 7.1 to show that to construct these polarized parts it is necessary and sufficient to construct the scalar component of the trace on $\partial \Omega_{1}$ of the amplitude $u_{n, n_{1}}$, that is to say, $\mu_{n}$.

iii) The analysis of paragraphs 7.1.5 and 7.1.6 tells us that this trace has to satisfy equation (150). In other words, $\mu_{n}$ depends on the unknown $\mu_{n+1}$ and the known functions $\mu_{n-1}$ (and possibly of $\widetilde{g}$ ). More precisely $\mu_{n}$ depends on the $\mu_{n+1}\left(\underline{\mathbf{t}}^{k}, \underline{\mathbf{x}}^{k}\right)$, for $k=1, \ldots, \min (n+1, M)$. Then applying again equation (150) but for $\mu_{n+1}$ we obtain that $\mu_{n+1}$ depends on $\mu_{n}$ and $\mu_{n+2}$. With more details, the $\mu_{n+1}\left(\underline{\mathbf{t}}^{k}, \underline{\mathbf{x}}^{k}\right)$, for $k=1, \ldots, \min (n+1, M)$ admits the following dependencies :

$$
\begin{array}{l|c|c}
\mu_{n}\left(\underline{\mathbf{t}}^{k}, \underline{\mathbf{x}}^{k}\right) & \mu_{n+2}\left(\underline{\mathbf{t}}^{k}, \underline{\left.\underline{\mathbf{x}}^{k}\right)}\right. \\
\mu_{n+1}(\underline{\mathbf{t}}, \underline{\mathbf{x}}) & k=1, \ldots, \underline{\min } & k=2, \ldots, \underline{\min } \\
\mu_{n+1}\left(\underline{\mathbf{t}}^{2}, \underline{\mathbf{x}}^{2}\right) & k=2, \ldots, \underline{\min } & k=3, \ldots, \underline{\min } \\
\vdots & \vdots & \vdots \\
\mu_{n+1}\left(\underline{\mathbf{t}}^{\min }, \underline{\mathbf{x}}^{\min }\right) & k \in \emptyset & k \in \emptyset \text { or } k=M
\end{array}
$$

where we denoted $\underline{\min }:=\min (n, M), \min :=\min (n+1, M)$ and $\overline{\min }=\min (n+2, M)$. Let us study the dependency on $\mu_{n}$. The worst term is $\mu_{n}(\underline{\mathbf{t}}, \underline{\mathbf{x}})$, indeed the other terms will be eliminate before $\mu_{n}(\underline{\mathbf{t}}, \underline{\mathbf{x}}$ ) (by the same arguments) and are harmless. Using equation (151), we obtain that $\mu_{n}(\underline{\mathbf{t}}, \underline{\mathbf{x}})$ in fact depends on $\mu_{n+1}\left(\underline{\mathbf{t}}^{k}, \underline{\mathbf{x}}^{k}\right)$, for $k=2, \ldots$, min. But the tabular (170) tells us that these traces depend, in fact, on $\mu_{n}\left(\underline{\mathbf{t}}^{k}, \underline{\mathbf{x}}^{k}\right)$ for $k=2, \ldots, \underline{\min }$. As a consequence, $\mu_{n+1}(\underline{\mathbf{t}}, \underline{\mathbf{x}})$ can be expressed in terms of the $\mu_{n}\left(\underline{\underline{t}}^{k}, \underline{\mathbf{x}}^{k}\right)$ for $k=2, \ldots, \underline{\min }$. Repeating the same argument we obtain that $\mu_{n+1}(\underline{\mathbf{t}}, \underline{\mathbf{x}})$ can be expressed in terms of the $\mu_{n}\left(\underline{\mathbf{t}}^{k}, \underline{\mathbf{x}}^{k}\right)$ for $k=3, \ldots, \underline{\text { min. }}$. Then if we repeat $\underline{\min }$ times this argument, we obtain that the $\mu_{n+1}\left(\underline{\mathbf{t}}^{k}, \underline{\mathbf{x}}^{k}\right)$ for $k=1, \ldots, \min$ (and consequently $\mu_{n}$ ) can be expressed in terms of $\mu_{n+2}\left(\underline{\mathbf{t}}^{k}, \underline{\mathbf{x}}^{k}\right)$ for $k>2$ only.

We thus repeat exactly the same reasoning for $\mu_{n+2}\left(\underline{\mathbf{t}}^{k}, \underline{\mathbf{x}}^{k}\right)$ to show that it can be expressed in terms of the $\mu_{n+3}\left(\underline{\mathbf{t}}^{k}, \underline{\mathbf{x}}^{k}\right)$ for $k>3$ and so on to determine $\mu_{n}$ in terms of $\widetilde{g}$ and $\mu_{n-1}$ (up to some compositions by the operators $\mathbb{T}$ and $\Lambda$ ) only, as it has already been made in paragraphs 7.1.6 and 7.3.1. This concludes the construction of the trace $\mu_{n}$ and as a consequence, the construction of the amplitudes linked to the loop's indices of order $n$.

$i v$ ) The construction of the other polarized part of the oscillating (or equivalently of the $\mathbb{P}_{e v 1} U$ (resp. $\left.\mathbb{P}_{e v 2} U\right)$ for the evanescent amplitudes for the side $\partial \Omega_{1}$ (resp. $\left.\partial \Omega_{2}\right)$ ) is then easy. Indeed these amplitudes are linked to frequencies for which the uniform Kreiss-Lopatinskii condition holds and we can use the "tree" structure of the frequency set to conclude (see [4] for a precise construction).

8. Proof of the main result. With Theorem 28 in hand, it is now easy to show Theorem 14 . We argue by contradiction. We thus assume that the corner problem (1) is weakly (or strongly) well-posed in the sense that there exists a positives integers $K, K_{1}$ and $K_{2}$ such that the corner problem (1) admits a solution satisfying the energy estimate:

$$
\|u\|_{L^{2}\left(\Omega_{T}\right)}^{2}+\left\|u_{\mid x_{1}=0}\right\|_{L^{2}\left(\partial \Omega_{1, T}\right)}^{2}+\left\|u_{\mid x_{2}=0}\right\|_{L^{2}\left(\partial \Omega_{2, T}\right)}^{2} \leq C_{T}\left(\|f\|_{H^{K}\left(\Omega_{T}\right)}^{2}+\left\|g_{1}\right\|_{H^{K_{1}\left(\partial \Omega_{1, T}\right)}}^{2}+\left\|g_{2}\right\|_{H^{K_{2}\left(\partial \Omega_{2, T}\right)}}^{2}\right),
$$

for $T>0$ if $\beta \geq 1$ and $0<T<T_{\max }$ if $\beta<1$. According to Theorem 28 , for any $M \in \mathbb{N}$ one can always construct a truncated geometric optics expansions for the corner problem (47). Here we only keep the $K+2$ first terms in the truncated expansion. It is given by:

$$
\begin{aligned}
u_{a p p}^{\varepsilon} & :=\sum_{n=0}^{K+1} \sum_{k \in \mathscr{I}_{o s}} \varepsilon^{n} e^{\frac{i}{\varepsilon} \varphi_{k}(t, x)} u_{n, k}(t, x) \\
& +\sum_{n=0}^{K+1} \sum_{k \in \Re_{1}} \varepsilon^{n} e^{\frac{i}{\varepsilon} \psi_{k, 1}\left(t, x_{2}\right)} U_{n, k, 1}\left(t, x, \frac{x_{1}}{\varepsilon}\right)+\sum_{n=0}^{K+1} \sum_{k \in \Re_{2}} \varepsilon^{n} e^{\frac{i}{\varepsilon} \psi_{k, 2}\left(t, x_{1}\right)} U_{n, k, 2}\left(t, x, \frac{x_{2}}{\varepsilon}\right) .
\end{aligned}
$$


Moreover one can always choose $g$ in such a way that the leading order of $u_{a p p}^{\varepsilon}$ is not identically zero. Let $u^{\varepsilon}$ be the solution of (47). The error $u^{\varepsilon}-u_{a p p}^{\varepsilon}$ satisfies the corner problem :

$$
\begin{cases}L(\partial)\left(u^{\varepsilon}-u_{a p p}^{\varepsilon}\right)=f^{\varepsilon}, & \text { in } \Omega_{T}, \\ B_{1}\left(u^{\varepsilon}-u_{a p p}^{\varepsilon}\right)_{\mid x_{1}=0}=0, & \text { on } \partial \Omega_{1, T}, \\ B_{2}\left(u^{\varepsilon}-u_{a p p}^{\varepsilon}\right)_{\mid x_{2}=0}=0, & \text { on } \partial \Omega_{2, T} \\ \left(u^{\varepsilon}-u_{\text {app }}^{\varepsilon}\right)_{\mid t \leq 0}=0, & \text { on } \Omega,\end{cases}
$$

where :

$$
\begin{aligned}
f^{\varepsilon} & :=\varepsilon^{K+1}\left[\sum_{k \in \mathscr{I}_{o s}} e^{i \frac{\varphi_{k}}{\varepsilon}} L(\partial) u_{K+1, k}\right. \\
& \left.+\sum_{k \in \Re_{1}} e^{i \frac{\psi_{k, 1}}{\varepsilon}}\left(L(\partial) U_{K+1, k, 1}\right)_{\mid X_{1}=\frac{x_{1}}{\varepsilon}}+\sum_{k \in \Re_{2}} e^{i \frac{\psi_{k, 2}}{\varepsilon}}\left(L(\partial) U_{K+1, k, 2}\right)_{\mid X_{2}=\frac{x_{2}}{\varepsilon}}\right] .
\end{aligned}
$$

From the energy estimate (171) we have:

$$
\left\|u^{\varepsilon}-u_{a p p}^{\varepsilon}\right\|_{L^{2}\left(\Omega_{T}\right)} \leq\left\|f^{\varepsilon}\right\|_{H^{K}\left(\Omega_{T}\right)} .
$$

It is then easy to see from its expression that $f^{\varepsilon}$ is $O(\varepsilon)$ in $H^{K}\left(\Omega_{T}\right)$ (the worst term to handle with is the one for which all the derivatives fall on the terms $e^{i \frac{\varphi_{k}}{\varepsilon}}, e^{i \frac{\psi_{k, 1}}{\varepsilon}}$ or $\left.e^{i \frac{\psi_{k, 2}}{\varepsilon}}\right)$. Consequently the error $u^{\varepsilon}-u_{a p p}^{\varepsilon}$ is $O(\varepsilon)$ in $L^{2}\left(\Omega_{T}\right)$. Using the fact that $u^{\varepsilon}$ is a solution of (47) we obtain that:

$$
\left\|u^{\varepsilon}\right\|_{L^{2}\left(\Omega_{T}\right)} \leq\left\|g^{\varepsilon}\right\|_{H^{K_{1}\left(\Omega_{T}\right)}}
$$

from which we deduce that $u^{\varepsilon}$ is $O\left(\varepsilon^{M-K_{1}}\right)$ in $L^{2}\left(\Omega_{T}\right)$. We choose $M>K_{1}$, by the triangle inequality and inequalities (174) and (175), it follows that $u_{a p p}^{\varepsilon}$ is at least $O(\varepsilon)$ which is a contradiction with the fact that $u_{0, n_{1}}$ is nonzero.

\section{Examples, conclusion and conjectures.}

9.1. Examples. Of course Theorem 14 to the corner problem (2) even if it may seem a bit abusive. Indeed, Theorem 14 needs strict hyperbolicity while the corner problem (2) is clearly not strictly hyperbolic. However, in the proof of Theorem 14, the only points where we used the hyperbolicity hypothesis were to establish the block structure and to use Lax Lemma. It can be shown that these points are still true for geometrically regular hyperbolic systems (see [16] for a precise definition) as soon as we are away from crossing points, which is the case for (2) under this choice of the source term.

A strictly hyperbolic example for which Theorem 14 applies is due to [19, paragraph 7]. In this example, the authors construct a system whose characteristic variety is composed of two intersecting ellipses choosen in such a configuration that a loop exists. The construction is the following a first ellipse is fixed and three points $A, B, D$ are chossen on this ellipse in such a way that:

- $\widehat{D A B}$ is a right angle

- The group velocity in $A$ is incoming-outgoing while the group velocities in $B$ and $D$ are outgoingincoming

This determines a unique $C$ such that $A B C D$ is a rectangle. Then we choose a second ellipse passing through $C$ such that the group velocity in this point is incoming-outgoing. Without loss of generality the loop can be constructed in the half space $\left\{\xi_{2}<0\right\}$, to make sure that the velocity along the boundary has the good sign. Then one chooses a boundary condition to make sure that the associated corner problem is in the $W R$ class. This point can be easily done because at this stage of the construction the system is composed of two decoupled subsystems.

To obtain a strictly hyperbolic system, it is sufficient to make the perturbation described in [19, paragraph 9]. The associated perturbed operator is strictly hyperbolic and remains in $W R$ class, because this class is stable by small perturbations (see [5]). 
9.2. Conclusion and conjectures. In this article, we constructed the rigorous geometric optics expansion for a corner problem for which the uniform Kreiss-Lopatinskii condition breaks down on a selfinteraction frequency. We have shown that it was always possible to choose the source term with support close enough of the corner problem to makes sure that the associated geometric optics expansion suffers $M+1$ amplifications, before a fixed time $T$, compared to the source term. $M$ can be arbitrarily big, so this leads to the fact that such a corner problem can not be weakly well-posed because it suffers an arbitrarily big number of losses of derivatives and thus present an Hadamard's instability. In terms of well-posedness it is the worst possible situation.

As a consequence we shown that for hyperbolic corner problem a weak instability can be repeated an arbitrarily number of times to cause a violent instability which differs from the degeneracy of the KreissLopatinskii condition.

Nevertheless in the author opinion systems admitting a loop are not so widespread at least for small values of $N$. Indeed, loops can not appear if $N=2$ (see [19]) and at the present time the examples in this article are, in the author knowledge, the only methods to construct a system with a loop (in particular we do not know any physical example). But it also seems reasonable to think that for large values of $N$ loops are much more frequent. Indeed when $N$ becomes large the geometry of the characteristic variety of the system becomes really complicated and we can imagine that more the geometry is more there are possibilities to have loops.

However obtain a more rigorous answer about the occurence of loops (or a full characterization of systems with loops) is a important question in the study of hyperbolic initial boundary value problems in the quarter space which is left for future studies.

In this article we treated a very particular kind of degeneracy of the uniform Kreiss-Lopatinskii condition and many others are possible. In these cases, one should be more optimistic. Let us formulate the following reasonable conjectures.

First of all let us stress that when the failure of the uniform Kreiss-Lopatinskii condition happens away from the loop then the weak instability can not be repeated an arbitrarily number of times and thus the system should be weakly well-posed. More precisely:

$\diamond$ If the uniform Kreiss-Lopatinskii condition breaks down in the elliptic area, using the fact that evanescent modes for the side $\partial \Omega_{1}$ are not reflected on the side $\partial \Omega_{2}$, the amplification observed in the high frequency expansion for the boundary value problem in the half space should not be improved. So we believe that the leading order in the WKB expansion should be of order $\varepsilon^{0}$ with a source term in the interior of order $\varepsilon$ and a source term on the side $\partial \Omega_{1}$ of order $\varepsilon^{0}$. The associated corner problem should be weakly well-posed with an energy estimate reading:

$$
\|u\|_{L^{2}\left(\Omega_{T}\right)}^{2}+\left\|u_{\mid x_{1}=0}\right\|_{L^{2}\left(\partial \Omega_{1, T}\right)}^{2}+\left\|u_{\mid x_{2}=0}\right\|_{L^{2}\left(\partial \Omega_{2, T}\right)}^{2} \leq C_{T}\left(\|f\|_{L^{2}\left(\Omega_{T}\right)}^{2}+\left\|g_{1}\right\|_{H^{1}\left(\partial \Omega_{1, T}\right)}^{2}+\left\|g_{2}\right\|_{L^{2}\left(\partial \Omega_{2, T}\right)}^{2}\right) .
$$

$\diamond$ When the uniform Kreiss-Lopatinskii condition is violated in the mixed area in such a way that $\operatorname{ker} B_{1} \cap E_{1}^{s}(\zeta)=\operatorname{ker} B_{1} \cap E_{1}^{s, e}(\zeta)=\operatorname{span}\{e\}$, where $E_{1}^{s, e}(\zeta)$ denotes the "elliptic" component of $E_{1}^{s}(\zeta)$ (see [2] and [12]), then the same argument should apply. The conjecture is then that we have the same amplifications as for the boundary value problem in the half space. The expected energy estimate is:

$$
\|u\|_{L^{2}\left(\Omega_{T}\right)}^{2}+\left\|u_{\mid x_{1}=0}\right\|_{L^{2}\left(\partial \Omega_{1, T}\right)}^{2}+\left\|u_{\mid x_{2}=0}\right\|_{L^{2}\left(\partial \Omega_{2, T}\right)}^{2} \leq C_{T}\left(\|f\|_{H^{\frac{1}{2}}\left(\Omega_{T}\right)}^{2}+\left\|g_{1}\right\|_{H^{1}\left(\partial \Omega_{1, T}\right)}^{2}+\left\|g_{2}\right\|_{L^{2}\left(\partial \Omega_{2, T}\right)}^{2}\right),
$$

or

$$
\|u\|_{L^{2}\left(\Omega_{T}\right)}^{2}+\left\|u_{\mid x_{1}=0}\right\|_{L^{2}\left(\partial \Omega_{1, T}\right)}^{2}+\left\|u_{\mid x_{2}=0}\right\|_{L^{2}\left(\partial \Omega_{2, T}\right)}^{2} \leq C_{T}\left(\|f\|_{L^{2}\left(\Omega_{T}\right)}^{2}+\left\|g_{1}\right\|_{H^{1}\left(\partial \Omega_{1, T}\right)}^{2}+\left\|g_{2}\right\|_{L^{2}\left(\partial \Omega_{2, T}\right)}^{2}\right),
$$

depending of a technical assumption already discussed in [2].

$\diamond$ Finally when the uniform Kreiss-Lopatinskii condition fails in the hyperbolic region (but away from the loop) the conjecture is that the leading order in the geometric optics expansion is of order $\varepsilon^{0}$ for source terms of order $\varepsilon^{M}$, where $M$ denotes the number of time that a ray has been amplified. More precisely $M$ is defined by:

$$
M:=\max _{i \in \mathscr{I}_{o s}} \#\left(\Upsilon \cap L_{i}\right),
$$




\section{where}

All the previous conjectures are made under the assumption that the transport along the boundary spreads the information away from the corner. The energy estimates and the amplifications in the geometric optics expansions when the transport along the boundary sends the information to the corner are left for future studies.

\section{Appendix.}

Details of the computations to establish equations (118)-(119) and (120). Let us develop :

$$
\begin{aligned}
Q_{1}^{n_{3}} L(\partial)\left(I-P_{1}^{n_{3}}\right) u_{1, n_{3}} & =-\frac{\bar{\xi}_{2}}{\underline{\xi}_{2}} \beta_{1}^{-1} \beta_{2}^{-1}\left[Q_{1}^{n_{3}} R_{1}^{n_{3}} \mathscr{S}^{n_{3}} e\left(\partial_{t t}^{2} \mu_{0}-\frac{\tau}{\bar{\xi}_{2}} \partial_{t 2}^{2} \mu_{0}\right)\right. \\
& +Q_{1}^{n_{3}} A_{2} R_{1}^{n_{3}} \mathscr{S}^{n_{3}} e\left(\left(-\frac{1}{v_{n_{2}, 2}}+\frac{\beta_{2}^{-1}}{v_{n_{1}, 1}}\right) \partial_{t t}^{2} \mu_{0}+\beta_{1}^{-1} \beta_{2}^{-1} \partial_{t 2}^{2} \mu_{0}\right. \\
& \left.\left.-\frac{\tau}{\bar{\xi}_{2}}\left(\left(-\frac{1}{v_{n_{2}, 2}}+\frac{\beta_{2}^{-1}}{v_{n_{1}, 1}}\right) \partial_{t 2}^{2} \mu_{0}+\beta_{1}^{-1} \beta_{2}^{-1} \partial_{22}^{2} \mu_{0}\right)\right)\right]\left(\mathbf{t}_{3}, \mathbf{x}_{3}\right),
\end{aligned}
$$

where we used the fact that $Q_{1}^{n_{3}} A_{1} R_{1}^{n_{3}}=0$. We then use the relation between $Q_{1}^{n_{3}} R_{1}^{n_{3}} \mathscr{S}^{n_{3}} e$ and $Q_{1}^{n_{3}} A_{2} R_{1}^{n_{3}} \mathscr{S}^{n_{3}} e$ given in (116) to express $Q_{1}^{n_{3}} L(\partial)\left(I-P_{1}^{n_{3}}\right) u_{1, n_{3}}$ in terms of $Q_{1}^{n_{3}} R_{1}^{n_{3}} \mathscr{S}^{n_{3}} e$ only :

$$
\begin{aligned}
Q_{1}^{n_{3}} L(\partial)\left(I-P_{1}^{n_{3}}\right) u_{1, n_{3}}= & -\frac{\bar{\xi}_{2}}{\underline{\xi}_{2}} \beta_{1}^{-1} \beta_{2}^{-1} Q_{1}^{n_{3}} R_{1}^{n_{3}} \mathscr{S}^{n_{3}} e\left[\partial_{t t}^{2} \mu_{0}\left(1+\frac{\underline{\tau}}{\underline{\xi}_{2}}\left(\frac{1}{v_{n_{2}, 2}}-\frac{\beta_{2}^{-1}}{v_{n_{1}, 1}}\right)\right)\right. \\
& \left.-C_{3} \partial_{t 2}^{2} \mu_{0}+\partial_{22}^{2} \mu_{0} \beta_{1}^{-1} \beta_{2}^{-1} \frac{\underline{\tau}^{2}}{\bar{\xi}_{2} \underline{\xi_{2}}}\right]\left(\mathbf{t}_{3}, \mathbf{x}_{3}\right)
\end{aligned}
$$

$$
C_{3}:=\frac{\underline{\tau}}{\bar{\xi}_{2}}\left[1+\frac{\underline{\tau}}{\underline{\xi_{2}}}\left(\frac{1}{v_{n_{2}, 2}}-\frac{\beta_{2}^{-1}}{v_{n_{1}, 1}}\right)\right]+\frac{\underline{\tau}}{\underline{\xi}_{2}} \beta_{1}^{-1} \beta_{2}^{-1} .
$$

1606

$$
\begin{aligned}
Q_{2}^{n_{4}} L(\partial)\left(I-P_{2}^{n_{4}}\right) u_{1, n_{4}} & =-\frac{\bar{\xi}_{2}}{\bar{\xi}_{1}} \prod_{j=1}^{3} \beta_{j}^{-1}\left[Q_{2}^{n_{4}} R_{2}^{n_{4}} \mathscr{S}^{n_{4}} e\left(\partial_{t t}^{2} \mu_{0}-\frac{\underline{\tau}}{\bar{\xi}_{2}} \partial_{t 2}^{2} \mu_{0}\right)\right. \\
& +Q_{2}^{n_{4}} A_{1} R_{2}^{n_{4}} \mathscr{S}^{n_{4}} e\left(-A \partial_{t t}^{2} \mu_{0}-\prod_{j=1}^{3} \beta_{j}^{-1} \partial_{t 2}^{2} \mu_{0}\right. \\
& \left.\left.-\frac{\underline{\tau}}{\bar{\xi}_{2}}\left(-A \partial_{t 2}^{2} \mu_{0}-\prod_{j=1}^{3} \beta_{j}^{-1} \partial_{22}^{2} \mu_{0}\right)\right)\right]\left(\mathbf{t}_{4}, \mathbf{x}_{4}\right),
\end{aligned}
$$

where we recall that $A$ is defined in (74). We then use (117) to express this equation in terms of $Q_{2}^{n_{4}} R_{2}^{n_{4}} \mathscr{S}^{n_{4}} e$ only :

$$
Q_{2}^{n_{4}} L(\partial)\left(I-P_{2}^{n_{4}}\right) u_{1, n_{4}}=-\frac{\bar{\xi}_{2}}{\bar{\xi}_{1}} \prod_{j=1}^{3} \beta_{j}^{-1} Q_{2}^{n_{4}} R_{2}^{n_{4}} \mathscr{S}^{n_{4}} e\left[\partial_{t t}^{2} \mu_{0}\left(1+A \frac{\tau}{\bar{\xi}_{1}}\right)+C_{4} \partial_{t 2}^{2} \mu_{0}+\frac{\tau^{2}}{\bar{\xi}_{1} \bar{\xi}_{2}} \prod_{j=1}^{3} \beta_{j}^{-1} \partial_{22}^{2} \mu_{0}\right]\left(\mathbf{t}_{4}, \mathbf{x}_{4}\right)
$$

where $C_{4}$ is defined by :

$$
C_{4}:=-\frac{\tau}{\bar{\xi}_{2}}\left(1+A \frac{\tau}{\bar{\xi}_{1}}\right)+\frac{\tau}{\bar{\xi}_{1}} \prod_{j=1}^{3} \beta_{j}^{-1} .
$$

1615 Once again thanks to Lemma 30, we obtain that $C_{4}$ in fact reads $C_{4}=2 \frac{\tau}{\bar{\xi}_{1}} \prod_{j=1}^{3} \beta_{j}^{-1}$. As a consequence we obtain (120). 
Acknowledgments. The author acknowledges financial support from the European Research Council under the European Community's Seventh Framework Programme (FP7/2014-2019 Grant Agreement QUANTHOM 335410).

The author also wishes to thank all the members, academic as well as administrative, of the "Laboratoire de Mathématiques Jean Leray" of Nantes, and more specifically Jean-François Coulombel. Because the main part of this research has been made in this department.

\section{REFERENCES}

[1] M. Artola, A. Majda, Nonlinear development of instabilities in supersonic vortex sheets. I. The basic kink modes, Phys. D, 28 (1987), pp. 253-281.

[2] A. Benoit, Geometric optics expansions for linear hyperbolic boundary value problems and optimality of energy estimates for surface waves, Differential Integral Equations, 27 (2014), pp. 531-562, http://projecteuclid.org/euclid.die/ 1396558096.

[3] A. Benoit, Problèmes aux limites, optique géométrique et singularités, PhD thesis, Université de Nantes, 2015. https: //hal.archives-ouvertes.fr/tel-01180449v1.

[4] A. Benoit, Geometric optics expansions for hyperbolic corner problems, I: Self-interaction phenomenon, Anal. PDE, 9 (2016), pp. 1359-1418, doi:10.2140/apde.2016.9.1359, http://dx.doi.org/10.2140/apde.2016.9.1359.

[5] S. Benzoni-Gavage, F. Rousset, D. Serre, and K. Zumbrun, Generic types and transitions in hyperbolic initialboundary-value problems, Proc. Roy. Soc. Edinburgh Sect. A, 132 (2002), pp. 1073-1104.

[6] S. Benzoni-Gavage, D. Serre, Multidimensional hyperbolic partial differential equations, Oxford Mathematical Monographs, Oxford University Press, 2007.

[7] J.-F. Coulombel, Well-posedness of hyperbolic initial boundary value problems, J. Math. Pures Appl. (9), 84 (2005), pp. $786-818$.

[8] J.-F. Coulombel And O. Guès, Geometric optics expansions with amplification for hyperbolic boundary value problems: linear problems, Ann. Inst. Fourier (Grenoble), 60 (2010), pp. 2183-2233, http://aif.cedram.org/item?id=AIF_2010__ 60_6_2183_0

[9] R. Hersh, Mixed problems in several variables, J. Math. Mech., 12 (1963), pp. 317-334.

[10] H.-O. Kreiss, Initial boundary value problems for hyperbolic systems, Comm. Pure Appl. Math., 23 (1970), pp. $277-298$.

[11] P. D. LAX, Asymptotic solutions of oscillatory initial value problems, Duke Math. J., 24 (1957), pp. 627-646.

[12] V. Lescarret, Wave transmission in dispersive media, Math. Models Methods Appl. Sci., 17 (2007), pp. $485-535$.

[13] A. Majda, R. Rosales, A theory for spontaneous Mach stem formation in reacting shock fronts. I. The basic perturbation analysis, SIAM J. Appl. Math., 43 (1983), pp. 1310-1334.

[14] A. MARCou, Rigorous weakly nonlinear geometric optics for surface waves, Asymptot. Anal., 69 (2010), pp. 125-174.

[15] G. MÉTIVIER, The block structure condition for symmetric hyperbolic systems, Bull. London Math. Soc., 32 (2000), pp. 689702.

[16] G. MÉTIVIer And K. Zumbrun, Hyperbolic boundary value problems for symmetric systems with variable multiplicities, J. Differential Equations, 211 (2005), pp. 61-134, doi:10.1016/j.jde.2004.06.002, http://dx.doi.org/10.1016/j.jde.2004. 06.002 .

[17] S. OsHeR, Initial-boundary value problems for hyperbolic systems in regions with corners. I, Trans. Amer. Math. Soc., 176 (1973), pp. 141-164.

[18] S. Osher, Initial-boundary value problems for hyperbolic systems in regions with corners. II, Trans. Amer. Math. Soc., 198 (1974), pp. 155-175.

[19] L. Sarason And J. A. Smoller, Geometrical optics and the corner problem, Arch. Rational Mech. Anal., 56 (1974/75), pp. 34-69.

[20] M. Williams, Nonlinear geometric optics for hyperbolic boundary problems, Comm. Partial Differential Equations, 21 (1996), pp. 1829-1895. 